\title{
Axon Physiology
}

\author{
DOMINIQUE DEBANNE, EMILIE CAMPANAC, ANDRZEJ BIALOWAS, EDMOND CARLIER, AND \\ GISÈLE ALCARAZ
}

Institut National de la Santé et de la Recherche Médicale U.641 and Université de la Méditerranée, Faculté de Médecine Secteur Nord, Marseille, France

I. Introduction $\quad 556$

II. Organization of the Axon 557

A. Complexity of axonal arborization: branch points and varicosities 557

B. Voltage-gated ion channels in the axon 557

C. Ligand-gated receptors in the axon

III. Axon Development and Targeting of Ion Channels in the Axon 564

A. Axon initial segments

B. Nodes of Ranvier $\quad 565$

C. Axon terminals

IV. Initiation and Conduction of Action Potentials

A. Action potential initiation $\quad 566$

B. Conduction of action potentials along the axon 569

V. Functional Computation in the Axon 571

A. Activity-dependent shaping of the presynaptic action potential 571

B. Signal amplification along the axon $\quad 572$

C. Axonal integration (analog signaling) 573

VI. Propagation Failures

A. Geometrical factors: branch points and swellings $\quad 577$

$\begin{array}{ll}\text { B. Frequency-dependent propagation failures } & 578\end{array}$

C. Frequency-independent propagation failures 580

VII. Reflection of Action Potential Propagation 580

VIII. Spike Timing in the Axon 582

A. Delay imposed by axonal length

B. Delays imposed by axonal irregularities and ion channels 583

C. Ephaptic interactions and axonal spike synchronization 583

D. Electric coupling in axons and fast synchronization 584

IX. Activity-Dependent Plasticity of Axon Morphology and Function 585

A. Morphological plasticity

B. Functional plasticity

X. Pathologies of Axonal Function 587

A. Axonal diseases involving ion channels

B. Axonal diseases involving myelin $\quad 588$

XI. Concluding Remarks 588

A. Increased computational capabilities $\quad 588$

B. Future directions and missing pieces $\quad 589$

Debanne D, Campanac E, Bialowas A, Carlier E, Alcaraz G. Axon Physiology.

Axons are generally considered as reliable transmission cables in which stable propagation occurs once an action potential is generated. Axon dysfunction occupies a central position in many inherited and acquired neurological disorders that affect both peripheral and central neurons. Recent findings suggest that the functional and computational repertoire of the axon is much richer than traditionally thought. Beyond classical axonal propagation, intrinsic voltage-gated ionic currents together with the geomet-rical properties of the axon determine several complex operations that not only control signal processing in brain circuits but also neuronal timing and synaptic efficacy. Recent evidence for the implication of these forms of axonal computation in the short-term dynamics of neuronal communication is discussed. Finally, we review how neuronal activity regulates both axon morphology and axonal function on a long-term time scale during development and adulthood. 


\section{INTRODUCTION}

The axon (from Greek $\alpha^{\prime} \xi \omega \nu$, axis) is defined as a long neuronal process that ensures the conduction of information from the cell body to the nerve terminal. Its discovery during the 19th century is generally credited to the German anatomist Otto Friedrich Karl Deiters (147), who first distinguished the axon from the dendrites. But the axon initial segment was originally identified by the Swiss Rüdolf Albert von Kölliker (293) and the German Robert Remak (439) (for a detailed historical review, see Ref. 480). The myelin of axons was discovered by Rudolf Virchow (548), and Louis-Antoine Ranvier (433) first characterized the nodes or gaps that now bear his name. The functional role of the axon as the output structure of the neuron was initially proposed by the Spanish anatomist Santiago Ramón y Cajal $(429,430)$.

Two distinct types of axons occur in the peripheral and central nervous system (PNS and CNS): unmyelinated and myelinated axons, the latter being covered by a myelin sheath originating from Schwann cells in the PNS or oligodendrocytes in the CNS (Table 1). Myelinated axons can be considered as three compartments: an initial segment where somatic inputs summate and initiate an action potential; a myelinated axon of variable length, which must reliably transmit the information as trains of action potentials; and a final segment, the preterminal axon, beyond which the synaptic terminal expands (Fig. 1). The initial segment of the axon is not only the region of action potential initiation $(117,124,514)$ but is also the most reliable neuronal compartment, where full action potentials can be elicited at very high firing frequencies without attenuation (488). Bursts of spikes display minimal attenuation in the AIS compared with the soma $(488,561)$. The main axon is involved in the secure propagation of action potentials, but it is also able to integrate fluctuations in membrane potential originating from the somatodendritic region to modulate neurotransmitter release $(5,291,489)$. Finally, the axon terminal that is principally devoted to excitation-release coupling with a high fidelity (159) is also the subject of activity-dependent regulation that may lead to spike broadening (209).

Generally, axons from the CNS are highly ramified and contact several hundreds of target neurons locally or distally. But, the function of the axon is not purely limited to the conduction of the action potential from the site of initiation near the cell body to the terminal. Recent experimental findings shed new light on the functional and computational capabilities of single axons, suggesting that several different complex operations are specifically achieved along the axon. Axons integrate subthreshold synaptic potentials and therefore signal both analog and digital events. Drop of conduction or backward propagation (reflection) may occur at specific axonal branch points under certain conditions. Axonal geometry together with the biophysical properties of voltage-gated channels determines the timing of propagation of the output message in different axonal branches. In addition, axons link central neurons through gap junctions that allow ultra-fast network synchrony. Moreover, local shaping of the axonal action potential may subsequently determine synaptic efficacy during repetitive stimulation. These operations have been largely described in in vitro preparations of brain tissue, but evidence for these processes is still scarce in the mammalian brain in vivo. In this paper we review the different ways in which the properties of axons can control the transmission of electrical signals. In particular, we show how the axon deter-

TABLE 1. A short list of myelinated and unmyelinated axons in mammalian CNS and PNS

\begin{tabular}{|c|c|c|}
\hline CNS & Neuronal Type & Nomenclature/Remarks \\
\hline \multicolumn{3}{|c|}{ Myelinated axons } \\
\hline \multicolumn{3}{|l|}{ CNS } \\
\hline Retina & Ganglion cells & Optic nerve (unmyelinated proximally) \\
\hline Spinal cord & & Grey matter \\
\hline Hippocampus & CA1/CA3 pyramidal neurons & Alveus/fimbria \\
\hline Cortex & L5 pyramidal cell & Principal axon (unmyelinated collaterals) \\
\hline Cerebellum & Purkinje cell & principal axon (unmyelinated collaterals) \\
\hline \multicolumn{3}{|r|}{ P } \\
\hline Motor & Motoneurons & $\alpha, \gamma$ (in sciatic nerve) \\
\hline Sensory fibers & Dorsal root ganglion cell & Type Ia, Ib, II (muscle spindle and Golgi tendon organ) \\
\hline \multicolumn{2}{|c|}{ Unmyelinated axons } & Type III (also known as nociceptive A $\delta$ fibers) \\
\hline CNS & & \\
\hline Hippocampus & Granule cell (dentate gyrus) & Mossy fiber \\
\hline Hippocampus & CA3 pyramidal cell & Schaffer collaterals \\
\hline $\begin{array}{l}\text { Cerebellar } \\
\text { PNS }\end{array}$ & Granule cell & Parallel fiber \\
\hline Sensory & Dorsal root ganglion cell & Type IV (also known as nociceptive C-fibers) \\
\hline
\end{tabular}

CNS, central nervous system; PNS, peripheral nervous system. 


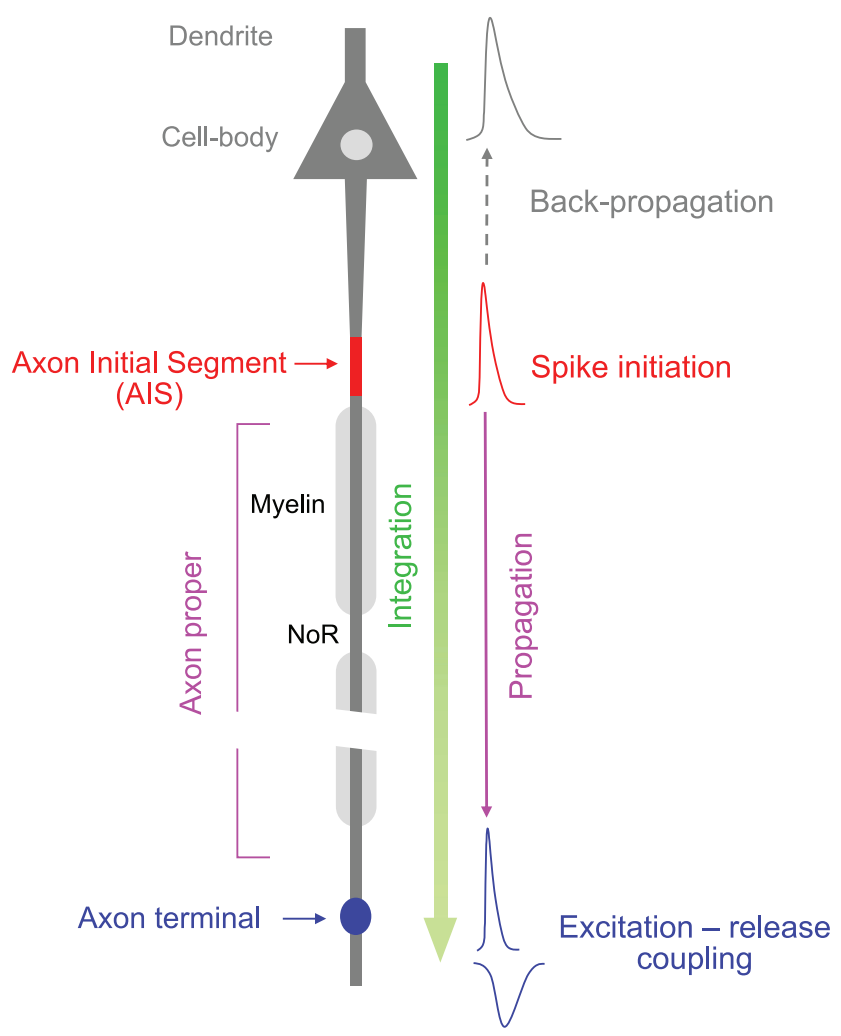

FIG. 1. Summary of axonal functions. A pyramidal neuron is schematized with its different compartments. Four major functions of the axon are illustrated (i.e., spike initiation, spike propagation, excitationrelease coupling, and integration). A spike initiates in the axon initial segment (AIS) and propagates towards the terminal where the neurotransmitter is released. In addition, electrical signals generated in the somatodendritic compartment are integrated along the axon to influence spike duration and neurotransmitter release (green arrow).

mines efficacy and timing of synaptic transmission. We also discuss recent evidence for long-term, activity-dependent plasticity of axonal function that may involve morphological rearrangements of axonal arborization, myelination, regulation of axonal channel expression, and fine adjustment of AIS location. The cellular and molecular biology of the axon is, however, not discussed in depth in this review. The reader will find elsewhere recent reviews on axon-dendrite polarity (36), axon-glia interaction (380, $381)$, myelin formation $(40,483)$, axonal transport $(138$, $250,405)$, and the synthesis of axonal proteins (210).

\section{ORGANIZATION OF THE AXON}

\section{A. Complexity of Axonal Arborization: Branch Points and Varicosities}

Axonal morphology is highly variable. Some axons extend locally ( $<1 \mathrm{~mm}$ long for inhibitory interneurons), whereas others may be as long as $1 \mathrm{~m}$ and more. The diameter of axons varies considerably (553). The largest axons in the mammalian PNS reach a diameter of $\sim 20 \mu \mathrm{m}$ [(264); but the biggest is the squid giant axon with a diameter close to $1 \mathrm{~mm}$ (575)], whereas the diameter of unmyelinated cortical axons in the mammalian brain varies between 0.08 and $0.4 \mu \mathrm{m}(48,559)$. The complexity of axonal arborization is also variable. In one extreme, the cerebellar granule cell axon possesses a single T-shaped branch point that gives rise to the parallel fibers. On the other, many axons in the central nervous system typically form an elaborate and most impressive tree. For instance, the terminal arbor of thalamocortical axons in layer 4 of the cat visual cortex contains 150-275 branch points (13). The complexity of axonal arborization is also extensive in cortical pyramidal neurons. Axons of hippocampal CA3 pyramidal cells display at least 100-200 branch points for a total axonal length of $150-300 \mathrm{~mm}$, and a single cell may contact 30,000-60,000 neurons (269, 325, 347). GABAergic interneurons also display complex axons. Hippocampal and cortical inhibitory interneurons emit an axon with a very dense and highly branched arborization (235). One obvious function of axonal divergence is to allow synchronous transmission to a wide population of target neurons within a given brain area. For instance, hippocampal basket cells synchronize the firing of several hundred principal cells through their divergent axon (118).

The second morphological feature of axons is the presence of a large number of varicosities (synaptic boutons) that are commonly distributed in an en passant, "string of beads" manner along thin axon branches. A single axon may contain several thousands of boutons $(235,325,411)$. Their size ranges between $\sim 1 \mu \mathrm{m}$ for thin unmyelinated axons $(482,559)$ and $3-5 \mu \mathrm{m}$ for large hippocampal mossy-fiber terminals $(61,482)$. Their density varies among axons, and the spacing of varicosities is comprised between $\sim 4$ and $\sim 6 \mu \mathrm{m}$ in unmyelinated fibers (481, 482).

\section{B. Voltage-Gated Ion Channels in the Axon}

Voltage-gated ion channels located in assigned subdomains of the axonal membrane carry out action potential initiation and conduction, and synaptic transmission, by governing the shape and amplitude of the unitary spike, the pattern of repetitive firing, and the release of neurotransmitters (Fig. 2). Recent reviews (310, 387, 540) have provided a detailed account of the voltage-gated ion channels in neurons, clearly illustrating the view that in the axon, the specific array of these channels in the various neuronal types adds an extra level of plasticity to synaptic outputs.

\section{Channels in the axon initial segment}

A) SODIUM CHANNELS. Variations in potential arising from somato-dendritic integration of multiple inputs culminate 


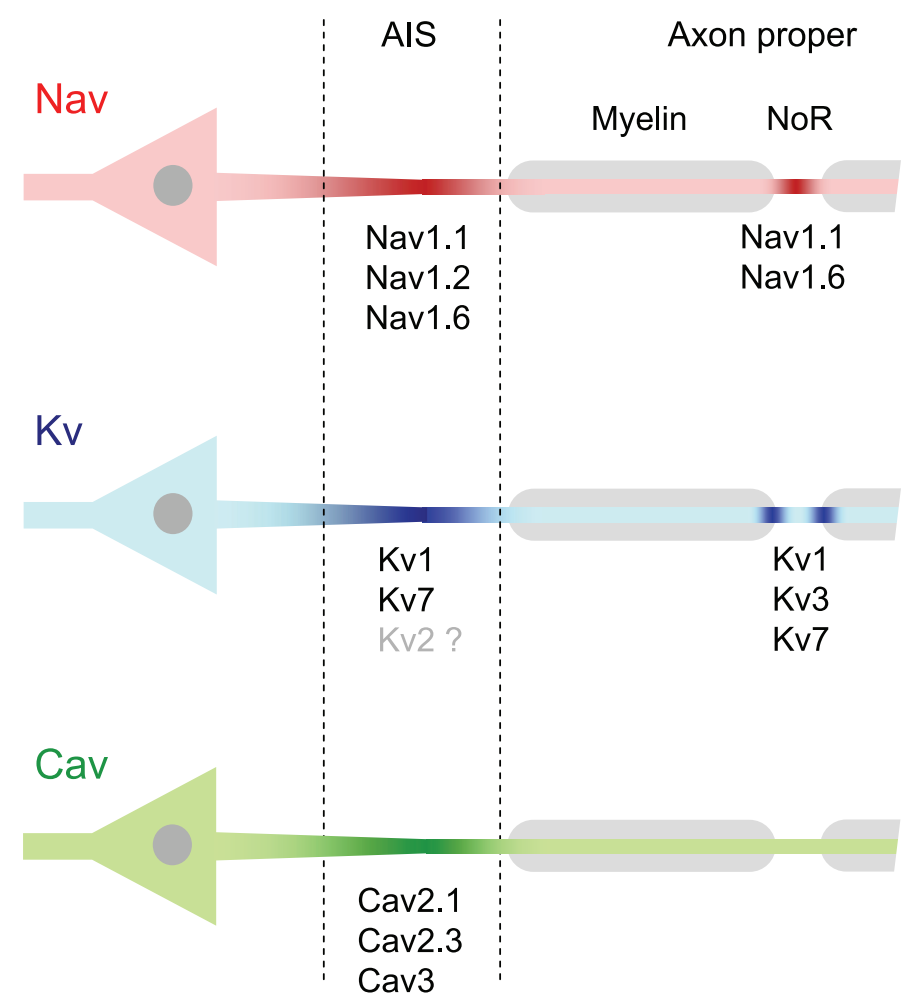

at the axon initial segment (AIS) where a suprathreshold resultant will trigger the action potential. This classical view relies on the presence of a highly excitable region in the initial segment of the axon (Fig. 3). Theoretical studies of action potential initiation have suggested that a 20 to 1,000 -fold higher density of sodium $\left(\mathrm{Na}^{+}\right)$channels in the axon relative to that found in the soma and dendrites is required to permit the polarity of spike initiation in the axon of the neuron $(157,346,373,434)$. The first evidence for concentration of $\mathrm{Na}^{+}$channels at the axon hillock and initial segment of retinal ganglion cells was obtained with the use of broad-spectrum $\mathrm{Na}^{+}$channel antibodies (564). After several fruitless attempts $(119,120)$, functional confirmation of the high concentration of $\mathrm{Na}^{+}$channels in the AIS was achieved only recently with the use of $\mathrm{Na}^{+}$ imaging $(193,290)$ and outside-out patch-clamp recordings from the soma and the axon (259). In these last studies, the largest $\mathrm{Na}^{+}$-dependent fluorescent signals or voltage-gated $\mathrm{Na}^{+}$currents were obtained in the AIS of cortical pyramidal neurons (Fig. $3, A$ and $B$ ). $\mathrm{Na}^{+}$current density is 34-fold greater in the AIS than in the soma (259). This estimation has been very recently confirmed for the Nav1.6 subunit detected in CA1 pyramidal neurons by a highly sensitive, quantitative electron microscope immunogold method (SDS-digested freeze-fracture replica-labeling; Ref. 333; Fig. 3C). The density of gold particles linked to Nav1.6 subunits measured by this method $\left(\sim 180 / \mu \mathrm{m}^{2}\right)$ is fully compatible with a previous estimate made by Kole and co-workers (290) in the AIS of L5 neurons where the density of $\mathrm{Na}^{+}$current amounts to

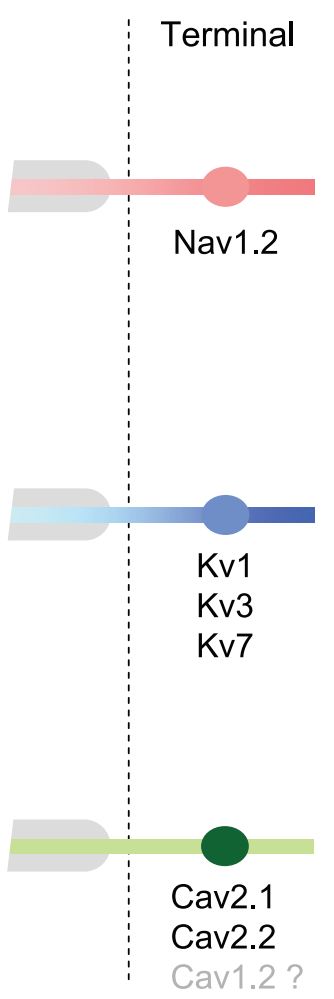

FIG. 2. Schematic representation of the distribution of sodium (top), potassium (middle), and calcium (bottom) channels in the different compartments of a myelinated axon. The cell body is symbolized by a pyramid shape (left). Channel densities are figured by the density of color. The myelin sheath is symbolized in gray. NoR, node of Ranvier; AIS, axon initial segment. Uncertain localizations are written in gray and accompanied by a question mark.

$2,500 \mathrm{pS} / \mu \mathrm{m}^{2}$ (i.e., $\sim 150$ channels $/ \mu \mathrm{m}^{2}$ given a $17 \mathrm{pS}$ unitary $\mathrm{Na}^{+}$channel conductance).

Three different isoforms of $\mathrm{Na}^{+}$channels, which drive the ascending phase of the action potential, are present at the AIS, namely, Nav1.1, Nav1.2, and Nav1.6. Nav1.1 is dominant at the AIS of GABAergic neurons (394), but it is also found in the AIS of retinal ganglion cells (542) and in spinal cord motoneurons (169; see Table 2 for details). With a few exceptions, its expression in interneurons is restricted to the proximal part of the AIS and displays little overlap with Nav1.6 that occupies the distal part (169, 332, 394, 542). Nav1.6 and Nav1.2 are principally associated with AIS of myelinated and unmyelinated axons, respectively, with Nav1.2 expressed first during development, then being gradually replaced by Nav1.6 concomitantly with myelination $(66,67)$. Although greatly diminished, the expression of Nav1.2 might persist in the AIS of adult neurons and is maintained in populations of unmyelinated axons. The two isoforms coexist in the AIS of L5 pyramidal neurons with a proximal distribution of Nav1.2 and a distal distribution of Nav1.6 (259). Sodium channels in the distal part of the AIS display the lowest threshold, suggesting that this polarized distribution could explain the unique properties of the AIS, including action potential initiation (principally mediated by Nav1.6) and backpropagation (largely supported by Nav1.2; Refs. 171, 259). A similar conclusion is drawn in CA1 pyramidal neurons where Nav1.6 sodium channels play a critical role for spike initiation (449). 
A
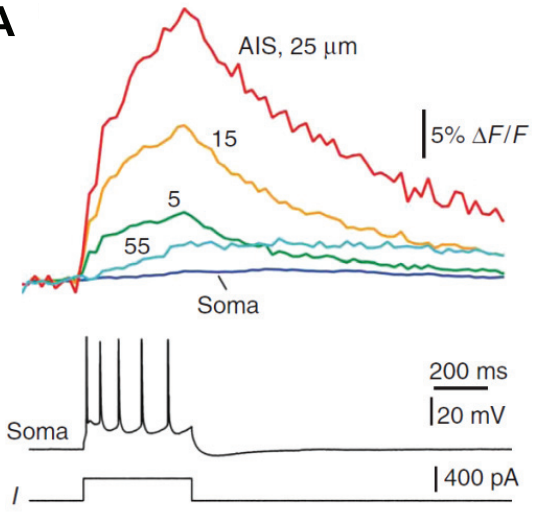

B
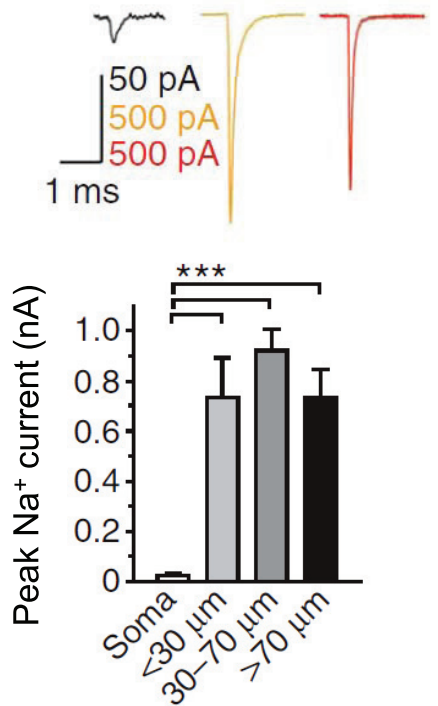

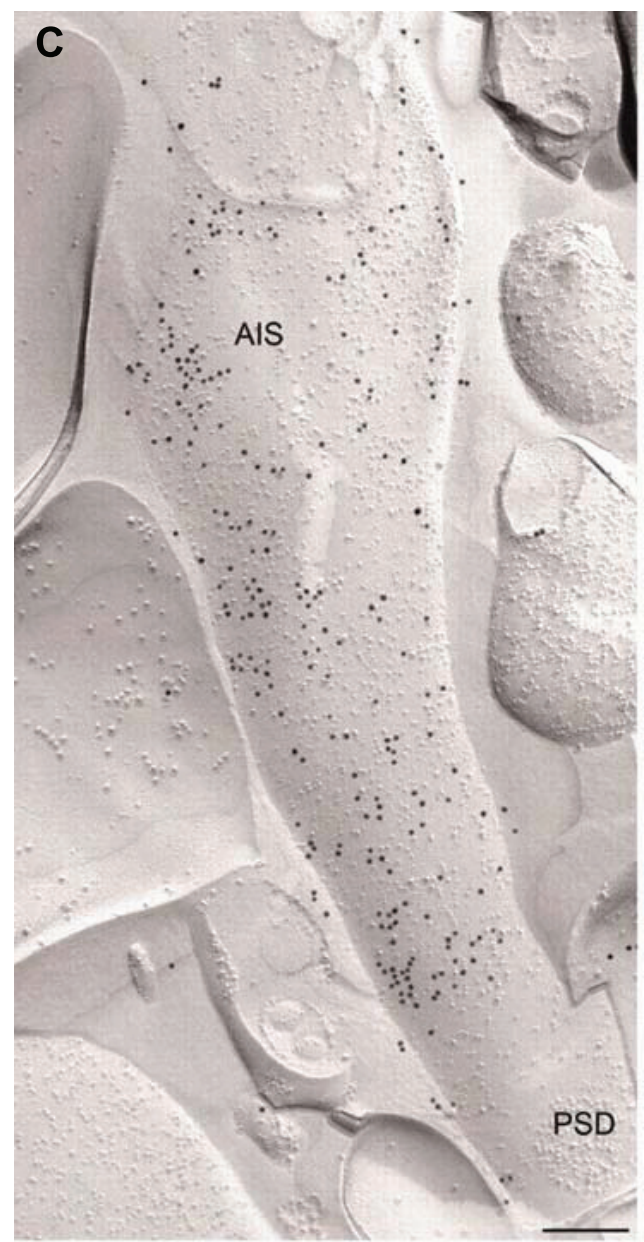

FIG. 3. High concentration of functional sodium channels at the AIS of cortical pyramidal neurons. $A$ : changes in intracellular $\mathrm{Na}^{+}$during action potentials are largest in the AIS. A L5 pyramidal neuron was filled with the $\mathrm{Na}^{+}$-sensitive dye SBFI and the variations in fluorescence measured at different distances from the axon hillock. The signal is larger in the AIS (25 $\mu \mathrm{m})$ and rapidly declines along the axon $(55 \mu \mathrm{m})$ or at proximal locations (5 $\mu \mathrm{m}$ or soma). [Adapted from Kole et al. (290), with permission from Nature Publishing Group.] $B: \mathrm{Na}^{+}$ channel density is highest at the AIS. Top: $\mathrm{Na}^{+}$currents evoked by step depolarizations $(30 \mathrm{~ms}$ ) from a holding potential of -100 to $+20 \mathrm{mV}$ in outside-out patches excised from the soma (black), AIS (orange, $39 \mu \mathrm{m}$ ), and axon (red, $265 \mu \mathrm{m})$. Bottom: average amplitude of peak $\mathrm{Na}^{+}$current obtained from different compartments. [From $\mathrm{Hu}$ et al. (259), with permission from Nature Publishing Group.] $C$ : high-resolution immunogold localization of the Nav1.6 subunit in AIS of CA1 pyramidal neuron. Gold particles labeling the Nav1.6 subunits are found at high density on the protoplasmic face of an AIS. Note the lack of immunogold particles in the postsynaptic density (PSD) of an axo-axonic synapse. [From Lorincz and Nusser (333), with permission from the American Association for the Advancement of Science.]
Nav channels generate three different $\mathrm{Na}^{+}$currents that can be distinguished by their biophysical properties, namely, 1) the fast-inactivating transient $\mathrm{Na}^{+}$current $\left(I_{\mathrm{NaT}}\right)$, the persistent $\mathrm{Na}^{+}$current $\left(I_{\mathrm{NaP}}\right)$, and the resurgent $\mathrm{Na}^{+}$current $\left(I_{\mathrm{NaR}}\right.$; i.e., a current activated upon repolarization; Ref. 427). The two last currents are activated at subthreshold or near-threshold, and they play a critical role in the control of neuronal excitability and repetitive firing (345). $I_{\mathrm{NaP}}$ is responsible for amplification of subthreshold excitatory postsynaptic potentials (EPSP) and is primarily generated in the proximal axon $(21,512) . I_{\mathrm{NaR}}$ is thought to facilitate reexcitation during repetitive firing and is generated in the AIS of cortical pyramidal neurons of the perirhinal cortex (99). $I_{\mathrm{NaR}}$ might be present all along the axon since a recent study indicates that this current shapes presynaptic action potentials at the Calyx of Held (246).

B) POTASSIUM CHANNELS. Potassium channels are crucial regulators of neuronal excitability, setting resting membrane potentials and firing thresholds, repolarizing action potentials, and limiting excitability. Specific voltage-gated potassium (Kv) conductances are also expressed in the AIS (see Fig. 2). Kv1 channels regulate spike duration in the axon (291, 490; Fig. 4A). Kv1.1 and Kv1.2 are most frequently associated at the initial segment of both excitatory and inhibitory cortical and hippocampal neurons (267, 332), and tend to be located more distally than Nav1.6. The current carried by these channels is indeed 10-fold larger in the distal part of the AIS than that measured in the soma (291). It belongs to the family of lowvoltage activated currents because a sizable fraction of the current is already activated at voltages close to the resting membrane potential $(291,490)$. These channels are also directly implicated in the high fidelity of action potential amplitude during burst firing (488).

Kv2.2 is present in the AIS of medial nucleus trapezoid neurons, where it promotes interspike hyperpolarization during repeated stimuli, thus favoring the extremely high frequency firing of these neurons (275). Kv7 channels (7.2 and 7.3), that bear the M-current (also called KCNQ channels), are also found in the AIS of many central neurons $(154,398,546)$. These channels are essential to 
TABLE 2. Subcellular localization of ion channels in CNS and PNS axons

\begin{tabular}{|c|c|c|c|c|}
\hline Localization & Channel & Neuronal Type & Region & Reference Nos. \\
\hline \multirow[t]{14}{*}{ AIS } & \multirow[t]{5}{*}{ Nav1.1 } & Fast-spiking PV+ interneuron & Hippocampus/cortex & 394 \\
\hline & & Purkinje cell & Cerebellum & 394 \\
\hline & & Interneuron & Olfactory bulb & 394 \\
\hline & & Ganglion cell & Retina & 542 \\
\hline & & & Spinal cord & 169 \\
\hline & \multirow[t]{2}{*}{ Nav1.2 } & Immature myelinated axons & Retina & 67 \\
\hline & & Pyramidal neuron & Neocortex & 259 \\
\hline & \multirow[t]{3}{*}{ Nav1.6 } & Ganglion cell & Retina & 67 \\
\hline & & Pyramidal neuron & Neocortex & 259 \\
\hline & & Pyramidal neuron & Hippocampus & 333 \\
\hline & Kv1.1 & Pyramidal neuron & Neocortex & 332 \\
\hline & Kv1.2 & Pyramidal neuron & Neocortex & 267 \\
\hline & Kv2.2 & Medial nucleus trapezoid & Brain stem & 275 \\
\hline & Kv7.2/7.3 & CA1 and CA3 pyramidal cells & hippocampus & 154 \\
\hline \multirow[t]{13}{*}{ NoR } & \multirow{4}{*}{ Nav1.1 } & & Spinal cord & 169 \\
\hline & & & Hippocampus & 169 \\
\hline & & & Brain stem & 169 \\
\hline & & & Cerebellum & 169 \\
\hline & Nav1.2 & Ganglion cell & Retina & 66 \\
\hline & \multirow[t]{4}{*}{ Nav1.6 } & & Sciatic nerve & 88 \\
\hline & & & Optic nerve & 88 \\
\hline & & Ganglion cell & Retina & 66 \\
\hline & & CA1 pyramidal cell & Hippocampus & 333 \\
\hline & \multirow{2}{*}{ Kv1.1/1.2 } & & Sciatic nerve & 585 \\
\hline & & & Spinal cord & 378,435 \\
\hline & Kv3.1b & Ganglion cell & Retina & 152 \\
\hline & Kv7.2/7.3 & & Sciatic nerve & 154 \\
\hline \multirow[t]{7}{*}{ AP (unmyelinated) } & \multirow[t]{2}{*}{ Nav1.2 } & Granule cell (MF axon) & Hippocampus & 223 \\
\hline & & Schaffer collaterals & Hippocampus & 223 \\
\hline & Kv1.2 & Schaffer collaterals & Hippocampus & 543 \\
\hline & Kv1.3 & Parallel fiber & Cerebellar & 305,543 \\
\hline & Kv3.3 & Granule cell (MF axon) & Hippocampus & 105 \\
\hline & Kv3.4 & Granule cell (MF axon) & Hippocampus & 105 \\
\hline & Kv7 & CA1 pyramidal cell & Hippocampus & 546 \\
\hline \multirow[t]{20}{*}{ AT } & Nav1.2 & Mossy fiber terminal & Hippocampus & 223 \\
\hline & Kv1.1/1.2 & Principal cells & Hippocampus & 551 \\
\hline & \multirow[t]{5}{*}{ Schaeffer collateral } & Boutons & Hippocampus & 371 \\
\hline & & Thalamo-cortical neurons & Neocortex & 311 \\
\hline & & Basket cell pinceau & Cerebellum & 319,504 \\
\hline & & Calyx of Held & Brain stem & $158,268,377$ \\
\hline & & Interneurons & Amygdala & 192 \\
\hline & Kv1.4 & Mossy fiber & Hippocampus & $126,478,543$ \\
\hline & \multirow[t]{3}{*}{ Kv3.1 } & Interneurons & Neocortex & 109,556 \\
\hline & & Calyx of Held & Brain stem & $177,268,377$ \\
\hline & & NTS neurons & Brain stem & 134 \\
\hline & \multirow[t]{2}{*}{ Kv3.2 } & Interneurons & Neocortex & 109 \\
\hline & & Purkinje cell & Cerebellum & 65 \\
\hline & Kv3.4 & Purkinje cell & Cerebellum & 65 \\
\hline & Kv7.2/7.3 & Mossy fiber & Hippocampus & 125 \\
\hline & Kv7.5 & Auditory neurons & Brain stem & 89 \\
\hline & \multirow{2}{*}{$\mathrm{HCN} 1$} & Basket cells & Hippocampus & $15,386,459$ \\
\hline & & Basket cells & Cerebellum & 334 \\
\hline & GIRK1 & Thalamo-cortical neurons & Cortex & 415 \\
\hline & GIRK1/2/3 & Granule cell & Cerebellum & 188 \\
\hline
\end{tabular}

the regulation of AP firing in hippocampal principal cells, where they control the resting membrane potential and action potential threshold (399, 473, 474, 579).

C) CALCIUM CHANNELs. The last players that have recently joined the AIS game are calcium channels (Fig. 2). Using two-photon $\mathrm{Ca}^{2+}$ imaging, Bender and Trussell (46) showed that T- and R-type voltage-gated $\mathrm{Ca}^{2+}$ channels are localized in the AIS of brain stem cartwheel cells. In this study, $\mathrm{Ca}^{2+}$ entry in the AIS of Purkinje cells and neocortical pyramidal neurons was also reported. These channels regulate firing properties such as spike-timing, burst-firing, and action potential threshold. The downregulation of T-type $\mathrm{Ca}^{2+}$ channels by dopamine receptor activation represents a powerful means to control action potential output (45). Using calcium imaging, pharmacological tools, and immunochemistry, a recent study reported the presence of P/Q-type (Cav2.1) and N-type (Cav2.2) $\mathrm{Ca}^{2+}$ channels in the AIS of L5 neocortical pyra- 
A

L5 pyramidal neuron
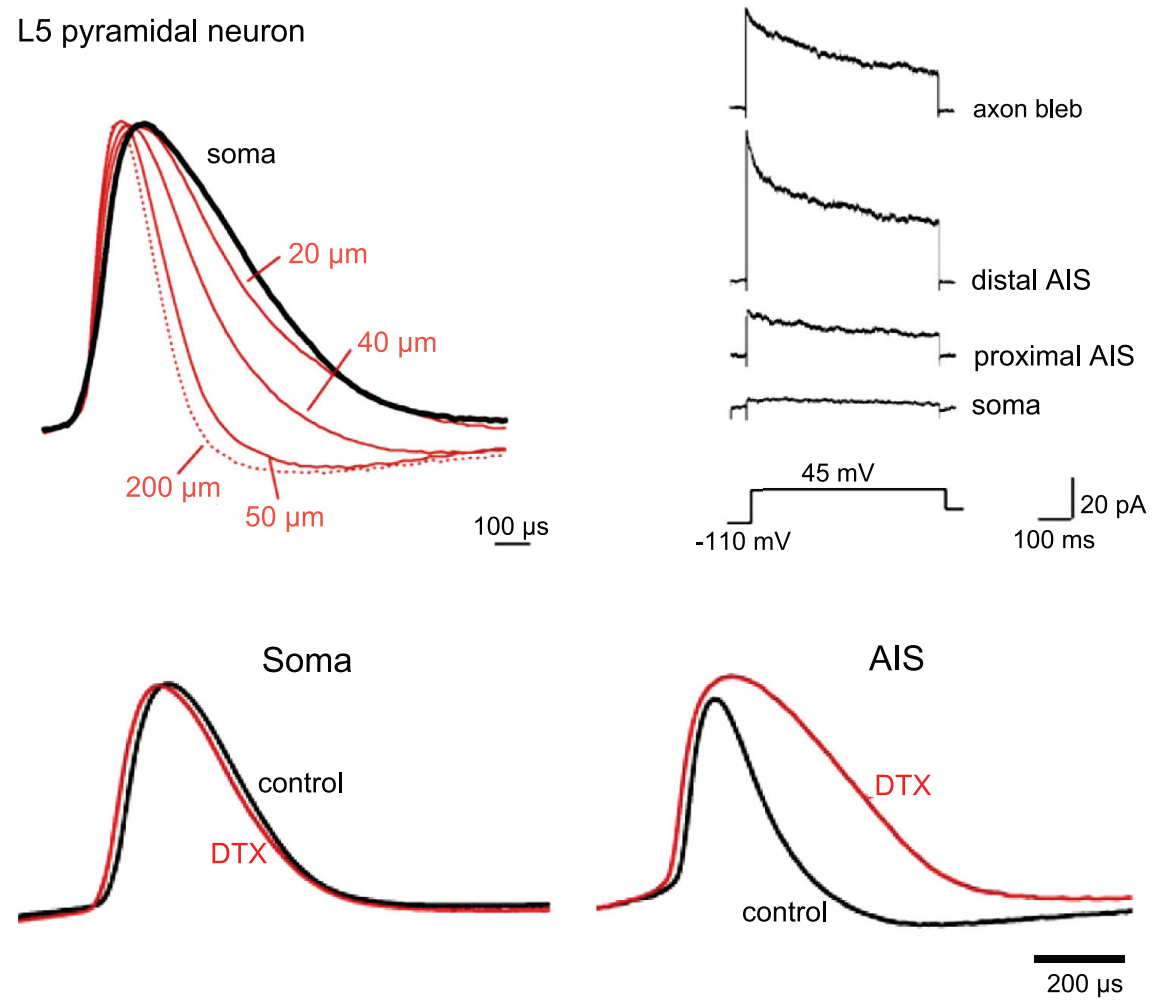

B

Mossy fiber terminal

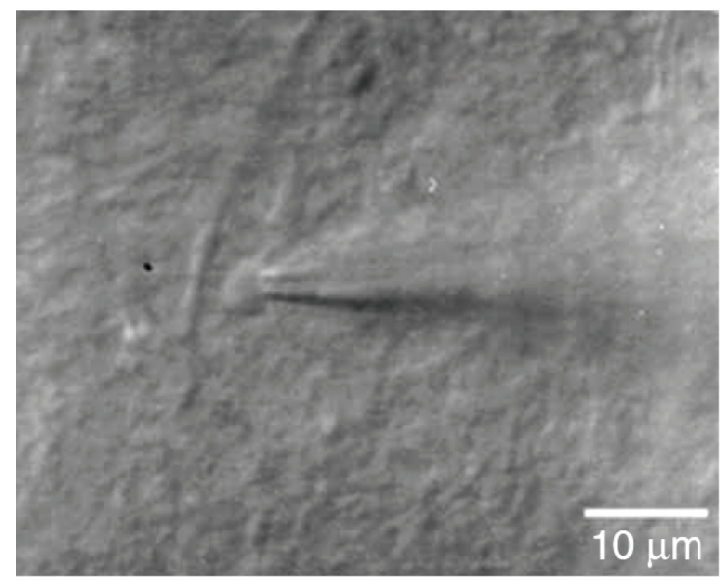

FIG. $4 . \mathrm{K}^{+}$channels determine $\mathrm{AP}$ duration in AIS of L5 pyramidal neuron and hippocampal mossy fiber terminals. A: DTX-sensitive $\mathrm{K}^{+}$channels determine spike duration in L5 pyramidal axons. Top left: superimposed AP traces recorded from the soma (black) and at the indicated axonal distances from the axon hillock (red). Top right: representative $\mathrm{K}^{+}$currents evoked by voltage steps from -110 to $+45 \mathrm{mV}$ in cellattached patches from the soma, proximal AIS (5-30 $\mu \mathrm{m})$, distal AIS (35-55 $\mu \mathrm{m}$ ), and axonal sites (up to $400 \mu \mathrm{m}$ ). Bottom: impact of 50-100 nM DTX-I on somatic (left) and axonal (right) APs before (black) and after DTX-I (red). Note the enlargement of AP in the AIS but not in the soma. [From Kole et al. (291), with permission from Elsevier.] $B$ : DTX-sensitive $\mathrm{K}^{+}$channels determine spike duration in mossy-fiber terminal. Left: mossy-fiber bouton approached with a patch pipette. [From Bischofberger et al. (58), with permission from Nature Publishing Group.] Top right: $\mathrm{K}^{+}$current activated in a mossy fiber bouton outside-out patch by pulses from -70 to $+30 \mathrm{mV}$ in the absence (control) and in the presence of $1 \mu \mathrm{M} \alpha$-dendrotoxin ( $\alpha$-DTX). Bottom right: comparison of the spike waveform in the soma and mossy fiber terminal (MF terminal) of a hippocampal granule cell. Note the large spike duration in the soma. [Adapted from Geiger and Jonas (209), with permission from Elsevier.]

midal neurons (577). These channels determine pyramidal cell excitability through activation of calcium-activated BK channels.

\section{Channels in unmyelinated axons}

In unmyelinated fibers, action potential conduction is supported by Nav1.2 sodium channels that are thought to be homogeneously distributed $(67,223,558)$.
At least five voltage-gated $\mathrm{K}^{+}$channel subunits are present in unmyelinated fibers (Table 2). Kv1.3 channels have been identified in parallel fiber axons of cerebellar granule cells $(305,543)$. The excitability of Schaffer collaterals is strongly enhanced by $\alpha$-dendrotoxin (DTX; a blocker of Kv1.1, Kv1.2, and Kv1.6) or margatoxin (MgTx; a blocker of $\mathrm{Kv} 1.2$ and $\mathrm{Kv1} 1.3$ ), indicating that $\mathrm{Kv1.2}$ is an important channel subunit for controlling excitability in these fibers (395). Hippocampal mossy fiber axons ex- 
press Kv3.3 and Kv3.4 channels (105). The Kv7 activator retigabine reduces excitability of C-type nerve fibers of human sural nerve (315). Kv7 channels determine excitability of pyramidal CA1 cell axons (546).

\section{Channels in the nodes of Ranvier}

In myelinated axons, conduction is saltatory and is made possible by the presence of hot spots of sodium channels in the node of Ranvier (Fig. 2). Two principal $\mathrm{Na}^{+}$channel isoforms are found in the nodes of PNS and CNS axons: Nav1.6 and Nav1.1 (88, 169, 333; see Table 2). In a recent study, Lorincz and Nusser (333) found that the density of Nav1.6 subunit in the node of Ranvier is nearly twice that observed in the AIS ( $\sim 350$ channels $\left./ \mu \mathrm{m}^{2}\right)$. Transient and persistent sodium currents have been identified in the node of myelinated axons $(47,166)$.

Saltatory conduction at nodes is secured by the juxtaparanodal expression of Kv1.1 and Kv1.2 channels, and by the nodal expression of Kv3.1b and Kv7.2/Kv7.3, which all concur to reduce reexcitation of the axon $(152,154$, $165,378,435,550,551,584,585)$. Other calcium- or sodium-activated potassium channels are encountered in the nodal region of myelinated axons (see Table 2).

\section{Channels in the axon terminals}

Axonal propagation culminates in the activation of chemical synapses with the opening of the presynaptic Cav2.1 and Cav2.2 calcium channels (Fig. 2). With the use of imaging techniques, the presence of calcium channels has been identified in en passant boutons of cerebellar basket cell axons where they presumably trigger transmitter release (330). Hot spots of calcium influx have also been reported at branch points (330). Although their function is not entirely clear, they may control signal transmission in the axonal arborization. In addition, Cav1.2 (L-type) calcium channels that are sparsely expressed all over hippocampal soma and dendrites are prominently labeled by immunogold electron microscopy in hippocampal axons and in mossy fiber terminals (531).

Functional sodium channels have been identified in presynaptic terminals of the pituitary (2), at the terminal of the calyx of Held $(260,320)$, and in hippocampal mossy fiber terminal (179). While Nav1.2 is probably the sole isoform of sodium channel expressed at terminals (in agreement with its exclusive targeting to unmyelinated portions of axons), terminal $\mathrm{Kv}$ channels exhibit a greater diversity (159). Kv1.1/Kv1.2 subunits dominate in many axon terminals (see Table 2 for details). Mossy fiber axons and boutons are enriched in Kv1.4 subunits (126, 478, $543)$ which determine the spike duration (Fig. $4 B$ ) and regulate transmitter release (209). The other main function of Kv1 channels is preventing the presynaptic terminal from aberrant action potential firing (158).
While Kv1 channels start to activate at low threshold, Kv3 conductances are typical high-voltage-activated currents. They have been identified in terminals of many inhibitory and excitatory neurons (see Table 2). Functionally, Kv3 channels keep action potential brief, thus limiting calcium influx and hence release probability (218).

Kv7 channels are also present in preterminal axons and synaptic terminals (see Table 2 for details). The specific M-channel inhibitor XE991 inhibits synaptic transmission at the Schaffer collateral input, whereas the Mchannel opener retigabine has the opposite effect, suggesting the presence of presynaptic Kv7 channels in Schaffer collateral terminals (546). It should be noted that these effects are observed in experimental conditions in which the M-current is activated, i.e., in the presence of a high external concentration of $\mathrm{K}^{+}$.

Other dampening channels such as the hyperpolarization-activated cyclic nucleotide-gated cationic (HCN) channels are expressed in the unmyelinated axon and in axon terminals (see Table 2). H-channels are also encountered at the calyx of Held giant presynaptic terminal (133) and in nonmyelinated peripheral axons of rats and humans $(30,225)$. The typical signature of $\mathrm{H}$-channels is also observed in cerebellar mossy fiber boutons recorded in vitro or in vivo (432). The postsynaptic function of $\mathrm{H}$ channels is now well understood, but their precise role in the preterminal axon and axon terminal is less clear. They may stabilize membrane potential in the terminal. For instance, the axons of cerebellar basket cells are particularly short, and any hyperpolarization or depolarization arising from the somatodendritic compartment may significantly change the membrane potential in the terminal and thus alter transmitter release. Thus stabilizing membrane potential in the terminal with a high density of HCN channels may represent a powerful means to prevent voltage shifts.

Besides voltage-gated conductances, axons and axon terminals also contain several ion-activated conductances including large-conductance, calcium-activated $\mathrm{BK}$ potassium channels (also called Maxi-K or Slo1 channels; Refs. 258, 287, 377, 423, 455), smallconductance calcium-activated SK potassium channels $(390,447)$, and sodium-activated $\mathrm{K}^{+}$channels $\left(\mathrm{K}_{\mathrm{Na}}\right.$, also called Slack channels or Slo2.2, Ref. 52) that are activated upon depolarization of the axon by the propagating action potential (Table 2). All these channels will also limit excitability of the nerve terminal by preventing uncontrolled repetitive activity.

G protein-gated inwardly rectifying potassium (GIRK) channels are also present at presynaptic terminals (Table 2). In the cortex and the cerebellum, these channels are functionally activated by $\mathrm{GABA}_{\mathrm{B}}$ receptors where they are thought to control action potential duration $(188,308)$. 


\section{Ligand-Gated Receptors in the Axon}

Axons do not contain only voltage- or metabolitegated ion channels but also express presynaptic vesicular release machinery (586) and many types of ligand-gated receptors including receptors to fast neurotransmitters and slow neuromodulators. We will focus here only on receptors that alter the excitability of the axon in physiological conditions.

\section{Receptors in the axon initial segment}

The axon initial segments of neocortical and hippocampal pyramidal neurons are particularly enriched in axo-axonic inhibitory contacts (499-501). A single axon initial segment receives up to 30 symmetrical synapses from a single axo-axonic (chandelier) GABAergic cell (500). Axon-initial segments contain a high concentration of the $\alpha 2$ subunit variant of the $\mathrm{GABA}_{\mathrm{A}}$ receptor (81). Axo-axonic synapses display a fast and powerful GABAergic current (340). The strategic location of GABAergic synapses on the AIS has generally been thought to endow axo-axonic cells with a powerful inhibitory action on the output of principal cells. However, this view has been recently challenged. Gabor Tamás and colleagues (522) recently discovered that axo-axonic synapses impinging on L2-3 pyramidal neurons may be in fact excitatory in the mature cortex. Importantly, the potassium-chloride cotransporter 2 (KCC2) is very weakly expressed in the AIS, and thus the reversal potential for GABA currents is much more depolarized in the axon than in the cell body (522). Similar conclusions have been drawn in the basolateral amygdala (566) and in hippocampal granule cells with the use of local uncaging of GABA in the different compartments of the neuron (285). However, a recent study using noninvasive techniques concludes that inhibitory postsynaptic potentials (IPSPs) may be hyperpolarizing throughout the entire neuron (211).

\section{Receptors in the axon proper}

$\mathrm{GABA}_{\mathrm{A}}$ receptors are not exclusively located in the AIS, but they have also been demonstrated in myelinated axons of the dorsal column of the spinal cord $(456,457)$ and in axonal branches of brain stem sensory neurons (545). Activation of these receptors modulates the compound action potential conduction and waveform. In some cases, propagation of antidromic spikes can be blocked by electrical stimulation of local interneurons (545). This effect is prevented by bath application of $\mathrm{GABA}_{\mathrm{A}}$ receptor channel blocker, suggesting that conduction block results from activation of $\mathrm{GABA}_{\mathrm{A}}$ receptors after the release of endogenous GABA. Similarly, GABA receptors have been identified in the trunk of peripheral nerves (79). However, the precise mode of physiological activation of these receptors remains unknown, and there is no clear evidence that GABA is released from oligodendrocytes or Schwann cells (307).

Monoamines regulate axonal properties in neurons from the stomatogastric ganglion of the crab or the lobster $(34,82,213,366)$. They also determine axonal properties in mammalian axons. For instance, subtype 3 of the serotonin receptor $\left(5-\mathrm{HT}_{3}\right)$ modulates excitability of unmyelinated peripheral rat nerve fibers (316).

Nicotinic acetylcholine receptors are encountered on unmyelinated nerve fibers of mammals where they modulate axonal excitability and conduction velocity (18, $314)$.

\section{Receptors in the periterminal axon and nerve terminals}

While the axon initial segment and the axon proper contain essentially $\mathrm{GABA}_{\mathrm{A}}$ receptors, the preterminal axon and nerve terminals are considerably richer and express many different modulatory and synaptic receptors (180). Only a subset of these receptors affects axonal excitability.

A) $\mathrm{GABA}_{\mathrm{A}}$ RECEPTORS. Although $\mathrm{GABA}_{\mathrm{B}}$ receptors are widely expressed on presynaptic excitatory and inhibitory terminals $(51,536)$, their action on periterminal and axonal excitability is slow and moderate. In contrast, high-conductance $\mathrm{GABA}_{\mathrm{A}}$ receptors control axonal excitability more accurately. Frank and Fuortes (197) first hypothesized modulation of transmitter release via axoaxonic inhibitory synapses to explain the reduction in monosynaptic transmission in the spinal cord (reviewed in Ref. 450). Based on the temporal correspondence between presynaptic inhibition and the depolarization of the primary afferent terminals, Eccles and co-workers (174) suggested that depolarization of the afferent was responsible for the inhibition of synaptic transmission. It was later shown that presynaptic inhibition is caused by a reduction in transmitter release $(168,175)$. Since this pioneering work, the primary afferent depolarization (PAD) has been demonstrated with axonal recordings and computational tools in many different sensory afferents including the cutaneous primary afferents of the cat (224), group Ib afferent fibers of the cat spinal cord (309, 312, $313)$, and sensory afferents of the crayfish (100-102). These studies and others $(132,515)$ indicate that activation of $\mathrm{GABA}_{\mathrm{A}}$ receptors produces a decrease in the amplitude of the presynaptic AP, thus decreasing transmitter release. Two mechanisms based on simulation studies have been proposed to account for presynaptic inhibition associated with PADs: a shunting mechanism (469) and inactivation of sodium channels (226). In the crayfish, the reduction in spike amplitude is mainly mediated by a shunting effect, i.e., an increase in membrane conductance due to the opening of $\mathrm{GABA}_{\mathrm{A}}$ receptors (102). The 
inactivation of sodium channels may add to the shunting effect for larger PADs.

Single action potentials evoked in cerebellar stellate and basket cells induce GABAergic currents measured in the soma, indicating that release of GABA regulates axonal excitability through $\mathrm{GABA}_{\mathrm{A}}$ autoreceptors (419). Application of the $\mathrm{GABA}_{\mathrm{A}}$ receptor agonist muscimol in the bath or locally to the axon modulates the excitability of hippocampal mossy fibers (452). The sign of the effect may be modulated by changing the intra-axonal $\mathrm{Cl}^{-}$concentration. Direct evidence for $\mathrm{GABA}_{\mathrm{A}}$ receptors on hippocampal granule cell axons has been provided unambiguously by Alle and Geiger (6) by the use of patch-clamp recordings from single mossy fiber boutons and local application of GABA. In mechanically dissociated CA3 pyramidal neurons from young rats, mossy fiber-derived release is strongly facilitated by stimulation of presynaptic $\mathrm{GABA}_{\mathrm{A}}$ receptors (273). This facilitation has been extensively studied by Ruiz and co-workers (451) with direct whole cell recordings from the mossy-fiber bouton. $\mathrm{GABA}_{\mathrm{A}}$ receptors modulate action potential-dependent $\mathrm{Ca}^{2+}$ transients and facilitate LTP induction (451).

B) GLYCINE RECEPTORS. In a similar way, glycine receptors may also control axonal excitability and transmitter release. At the presynaptic terminal of the calyx of Held, glycine receptors replace $\mathrm{GABA}_{\mathrm{A}}$ receptors as maturation proceeds (538). Activation of presynaptic glycine receptors produces a weakly depolarizing $\mathrm{Cl}^{-}$current in the nerve terminal and enhances synaptic release (537). The depolarization induces a significant increase in the basal concentration of $\mathrm{Ca}^{2+}$ in the terminal (24). Similar conclusions are reached in the ventral tegmental area where presynaptic glycine receptors lead to the facilitation of GABAergic transmission through activation of voltagegated calcium channels and intraterminal concentration of $\mathrm{Ca}^{2+}$ (573).

C) GLUTAMATE RECEPTORS. At least three classes of glutamate receptors are encountered at presynaptic release sites where they regulate synaptic transmission (412). Only a small fraction of these receptors regulates axonal excitability. In the CA1 region of the hippocampus, kainate produces a marked increase in spontaneous IPSCs. This effect might result from the direct depolarization of the axons of GABAergic interneurons (472). In fact, kainate receptors lower the threshold for antidromic action potential generation in CA1 interneurons.

NMDA receptors are encountered in many axons. They determine synaptic strength at inhibitory cerebellar synapses $(170,212)$, at granule cell-Purkinje cell synapse (54, 97), at L5-L5 excitatory connections (494), and at L2/3 excitatory synapses (127). However, recent studies indicate that axonal NMDA receptors do not produce significant depolarization or calcium entry in cerebellar stellate cells (111) and in L5 pyramidal cell axons (112) to significantly affect axonal excitability. In fact, NMDA receptors might modulate presynaptic release simply by the electrotonic transfer of the depolarization from the somatodendritic compartments to the axonal compartment (111, 112); see also sect. VC). However, such tonic change in the somatodendritic compartment of the presynaptic cell has not been observed in paired-recording when presynaptic NMDA receptors are pharmacologically blocked (494).

D) PURINE RECEPTORS. ATP and its degradation products, $\mathrm{ADP}$ and adenosine, are considered today as important signaling molecules in the brain (84). Classically ATP is coreleased from vesicles with acetylcholine (437) or GABA (274). However, a recent study indicates that ATP can also be relased by the axon in a nonvesicular manner through volume-activated anion channels (191). In fact, propagating action potentials cause microscopic swelling and movement of axons that may in turn stimulate volume-activated anion channels to restore normal cell volume through the release of water together with ATP and other anions.

Purinergic receptors are divided into three main families: $P 1$ receptors (G protein-coupled, activated by adenosine and subdivided into $\mathrm{A}_{1}, \mathrm{~A}_{2 \mathrm{~A}}, \mathrm{~A}_{2 \mathrm{~B}}$ and $\mathrm{A}_{3}$ receptors), $\mathrm{P} 2 \mathrm{X}$ receptors (ligand-gated, activated by nucleotides and subdivided into $\mathrm{P} 2 \mathrm{X}_{1-7}$ ), and $\mathrm{P} 2 \mathrm{Y}$ (G protein-coupled, activated by nucleotides and subdivided into $\mathrm{P} \mathrm{Y}_{1-14}$ ) (85). Purine receptors are found on axon terminals where they modulate transmitter release. For instance, activation of presynaptic $A_{1}$ receptor powerfully inhibits glutamate, but not GABA release, in the hippocampus $(529,574)$. In contrast, activation of presynaptic P2X receptor by ATP enhances GABA and glycine release in spinal cord (263, 442). $\mathrm{P} 2 \mathrm{X}_{7}$ receptors are expressed on developing axons of hippocampal neurons, and their stimulation promotes axonal growth and branching in cultured neurons (155).

\section{AXON DEVELOPMENT AND TARGETING OF ION CHANNELS IN THE AXON}

Neurons acquire their typical form through a stereotyped sequence of developmental steps. The cell initially establishes several short processes. One of these neurites grows very rapidly compared with the others and becomes the axon (161). The spatial orientation of the growing axon is under the control of many extracellular cues that have been reviewed elsewhere $(36,156)$. This section is therefore focused on the description of the major events underlying development and targeting of ion channels in the three main compartments of the axon.

\section{A. Axon Initial Segments}

In addition to its role in action potential initiation involving a high density of ion channels, the AIS might be 
also be defined by the presence of a specialized and complex cellular matrix, specific scaffolding proteins, and cellular adhesion molecules (393). The cellular matrix together with the accumulation of anchored proteins forms a membrane diffusion barrier $(375,563)$. This diffusion barrier plays an important role in preferentially segregating proteins into the axonal compartment. Recently, a cytoplasmic barrier to protein traffic has been described in the AIS of cultured hippocampal neurons (502). This filter allows entry of molecular motors of the kinesin-1 family (KIF5) that specifically carry synaptic vesicle proteins which must be targeted to the axon. The entry of kinesin-1 into the axon is due to the difference in the nature of microtubules in the soma and the AIS (294). Molecular motors (KIF17) that carry dendrite-targeted postsynaptic receptors cannot cross the axonal filter (19, $502,567)$. This barrier develops between 3 and 5 days in vitro (i.e., $\sim 1$ day after the initial elongation of the process that becomes an axon).

The scaffolding protein ankyrin G (AnkG) is critical for assembly of AIS and is frequently used to define this structure in molecular terms (233). The restriction of many AIS proteins within this small axonal region is achieved through their anchoring to the actin cytoskeleton via AnkG (296). AnkG is attached to the actin cytoskeleton via $\beta I V$ spectrin (49). Sodium channels, Kv7 channels, the cell adhesion molecule neurofascin-186 (NF-186), and neuronal cell adhesion molecules (NrCAM) are specifically targeted to the AIS through interaction with AnkG (154, 206, 245, 398). Furthermore, deletion of AnkG causes axons to acquire characteristics of dendrites with the appearance of spines and postsynaptic densities (244). While Nav and Kv7 channels are clustered through their interaction with AnkG, clustering of Kv1 channels in the AIS is under the control of the postsynaptic density 93 (PSD-93) protein, a member of the membrane-associated guanylate kinase (MAGUK) family (391). Some of the interactions between channels and AnkG are regulated by protein kinases. For instance, the protein kinase CK2 regulates the interaction between Nav and AnkG (75). But other factors might also control the development and targeting of $\mathrm{Na}^{+}$channels at the AIS. For instance, the sodium channel $\beta 1$ subunit determines the development of Nav1.6 at the AIS (73). The absence of the phosphorylated $\mathrm{I} \kappa \mathrm{B} \alpha$ at the AIS, an inhibitor of the nuclear transcription factor $-\kappa \mathrm{B}$, impairs sodium channel concentration (458).

The AIS may also contain axon specification signals (222). Cutting the axon of cultured hippocampal neurons is followed by axonal regeneration at the same site if the cut is $>35 \mu \mathrm{m}$ from the soma (i.e., the AIS is still connected to the cell body). In contrast, regeneration occurs from a dendrite if the AIS has been removed (222).

\section{B. Nodes of Ranvier}

During development, Nav1.2 channels appear first at immature nodes of Ranvier (NoR) and are eventually replaced by Nav1.6 (66). Later, Kv3.1b channels appear at the juxtaparanodal region, just before Kv1.2 channels (152). While targeting of ion channels at the AIS largely depends on intrinsic neuronal mechanisms, the molecular organization of the NoR and its juxtaparanodal region is mainly controlled by interactions between proteins from the axon and the myelinating glia $(310,393,413)$. For instance, in mutants that display abnormal myelin formation, Nav1.6 channels are dispersed or only weakly clustered in CNS axons $(66,276)$. In PNS axons, nodes are initiated by interactions between secreted gliomedin, a component of the Schwann cell extracellular matrix, and NF-186 (183). But once the node is initiated, targeting of ion channels at the NoR resembles that at the AIS. Accumulation of Nav channels at NoR also depends on AnkG (173). However, Kv1 clustering at the juxtaparanodal region of PNS axons depends on the cell adhesion molecules Caspr2 and TAG-1 that partly originate from the glia but not on MAGUKs $(257,413,414)$.

\section{Axon Terminals}

In contrast to the AIS and the NoR, much less is known about the precise events underlying development and targeting of ion channels in axon terminals. However, the trafficking of $\mathrm{N}$ - and $\mathrm{P} / \mathrm{Q}$-type $\mathrm{Ca}^{2+}$ channels to axon terminal and that of $\mathrm{GABA}_{\mathrm{B}}$ receptors illustrate the presence of specific targeting motifs on axonal terminal proteins. The $\mathrm{COOH}$-terminal region of the $\mathrm{N}$-type $\mathrm{Ca}^{2+}$ channel (Cav2.2) contains an amino acid sequence that constitutes a specific binding motif to the presynaptic protein scaffold, allowing their anchoring to the presynaptic terminal $(356,357)$. Furthermore, direct interactions have been identified between the t-SNARE protein syntaxin and N-type $\mathrm{Ca}^{2+}$ channels $(323,479)$. Deletion of the synaptic protein interaction (synprint) site in the intracellular loop connecting domains II and III of P/Q-type $\mathrm{Ca}^{2+}$ channels (Cav2.1) not only reduces exocytosis but also inhibits their localization to axon terminals (370).

One of the two subtypes of $\mathrm{GABA}_{\mathrm{B}}$ receptor $\left(\mathrm{GABA}_{\mathrm{B} 1 \mathrm{a}}\right)$ is specifically targeted to the axon (547). The $\mathrm{GABA}_{\mathrm{B} 1 \mathrm{a}}$ subunit carries two $\mathrm{NH}_{2}$-terminal interaction motifs, the "sushi domains" that are potent axonal targeting signals. Indeed, mutations in these domains prevent protein interactions and preclude localization of $\mathrm{GABA}_{\mathrm{B} 1 \mathrm{a}}$ subunits to the axon, while fusion of the wild-type $\mathrm{GABA}_{\mathrm{B} 1 \mathrm{a}}$ to mGluR1a preferentially redirects this somatodendritic protein to axons and their terminals (56).

In the pinceau terminal of cerebellar basket cells, HCN1 channels develop during the end of the second 
postnatal week (334). This terminal is particularly enriched in Kv1 channels (319), but the precise role of molecular partners and scaffolding proteins in clustering these channels remains unknown (392).

\section{INITIATION AND CONDUCTION OF ACTION POTENTIALS}

\section{A. Action Potential Initiation}

Determining the spike initiation zone is particularly important in neuron physiology. The action potential classically represents the final step in the integration of synaptic messages at the scale of the neuron $(41,514)$. In addition, most neurons in the mammalian central nervous system encode and transmit information via action potentials. For instance, action potential timing conveys significant information for sensory or motor functions (491). In addition, action potential initiation is also subject to many forms of activity-dependent plasticity in central neurons (493). Thus information processing in the neuronal circuits greatly depends on how, when, and where spikes are generated in the neuron.

\section{A brief historical overview}

Pioneering work in spinal motoneurons in the 1950s indicated that action potentials were generated in the AIS or possibly the first NoR $(124,187,202)$. Microelectrode recordings from motoneurons revealed that the action potential consisted of two main components: an "initial segment" (IS) component was found to precede the full action potential originating in the soma [i.e., the somatodendritic (or SD) component]. These two components could be isolated whatever the mode of action potential generation (i.e., antidromic stimulation, direct current injection, or synaptic stimulation), but the best resolution was obtained with the first derivative of the voltage. The IS component is extremely robust and can be isolated from the SD component by antidromic stimulation of the axon in a double-shock paradigm (124). For very short interstimulus intervals, the SD component fails but not the IS component. With simultaneous recordings at multiple axonal and somatic sites of the lobster stretch receptor neuron, Edwards and Ottoson (176) also reported that the origin of the electrical impulse occurred first in the axon, but at a certain distance from the cell body (176).

This classical view was challenged in the 1980s and 1990s with the observation that under very specific conditions, action potentials may be initiated in the dendrites (438). The development in the 1990s of approaches using simultaneous patch-pipette recordings from different locations on the same neuron was particularly precious to address the question of the site of action potential initiation $(514,516)$. In fact, several independent studies converged on the view that dendrites were capable of generating regenerative spikes mediated by voltage-gated sodium and/or calcium channels (220, 331, 462, 513, 565). The initiation of spikes in the dendrites (i.e., preceding somatic action potentials) has been reported in neocortical (513), hippocampal (220), and cerebellar neurons (431) upon strong stimulation of dendritic inputs. However, in many different neuronal types, threshold stimulations preferentially induce sodium spikes in the neuronal compartment that is directly connected to the axon hillock $(60,242,318,354,506,511,513,516)$. Thus the current rule is that the axon is indeed a low-threshold initiation zone for sodium spike generation. But the initiation site was precisely located only recently by direct recording from the axon.

\section{Initiation in the axon}

The recent development of techniques allowing loose-patch $(22,70,116,362,422)$ or whole cell recording $(291,355,463,489,561)$ from single axons of mammalian neurons, together with the use of voltage-sensitive dyes $(196,396,397)$ or sodium imaging $(46,193,290)$, provide useful means to precisely determine the spike initiation zone. These recordings have revealed that sodium spikes usually occur in the axon before those in the soma (Fig. 5, $A$ and $B$ ). More specifically, the initiation zone can be estimated as the axonal region where the advance of the axonal spike relative to the somatic spike is maximal (Fig. $5 C$ ). In addition, bursts of action potentials are generally better identified in the axon than in the cell body (355, $561)$.

In myelinated axons, action potentials are initiated at the AIS (22, 196, 283, 284, 396, 397, 488, 578). Depending on cell type, the initiation zone varies being located between 15 and $40 \mu \mathrm{m}$ from the soma. In layer 5 pyramidal neurons, the action potential initiation zone is located in the distal part of the AIS, i.e., at 35-40 $\mu \mathrm{m}$ from the axon hillock $(70,397,578)$. Similar estimates have been obtained in subicular pyramidal neurons with an AP initiation zone located at $\sim 40 \mu \mathrm{m}$ from the soma, beyond the AIS (119). The precise reason why the locus of axonal spike generation in myelinated fibers varies between the AIS and the first NoR is not known, but it may result from the heterogeneous distribution of Nav and Kv channels as well as the existence of ectopic zones for spike initiation $(410,580)$. In cerebellar Purkinje cell axons, the question was debated until recently. On the basis of latency differences between simultaneous whole cell somatic and cellattached axonal recordings, the action potential was found to be generated at the first NoR (at a distance of $\sim 75 \mu \mathrm{m}$; Ref. 116). However, in another study, it was concluded that spike initiation was located at the AIS (i.e., 
A
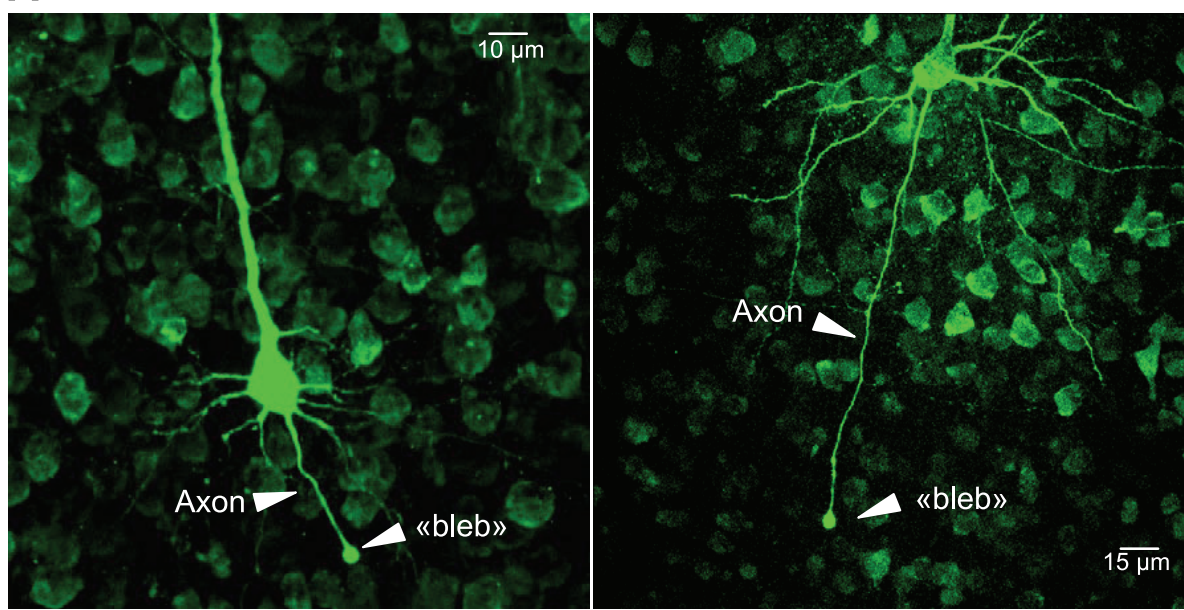

B

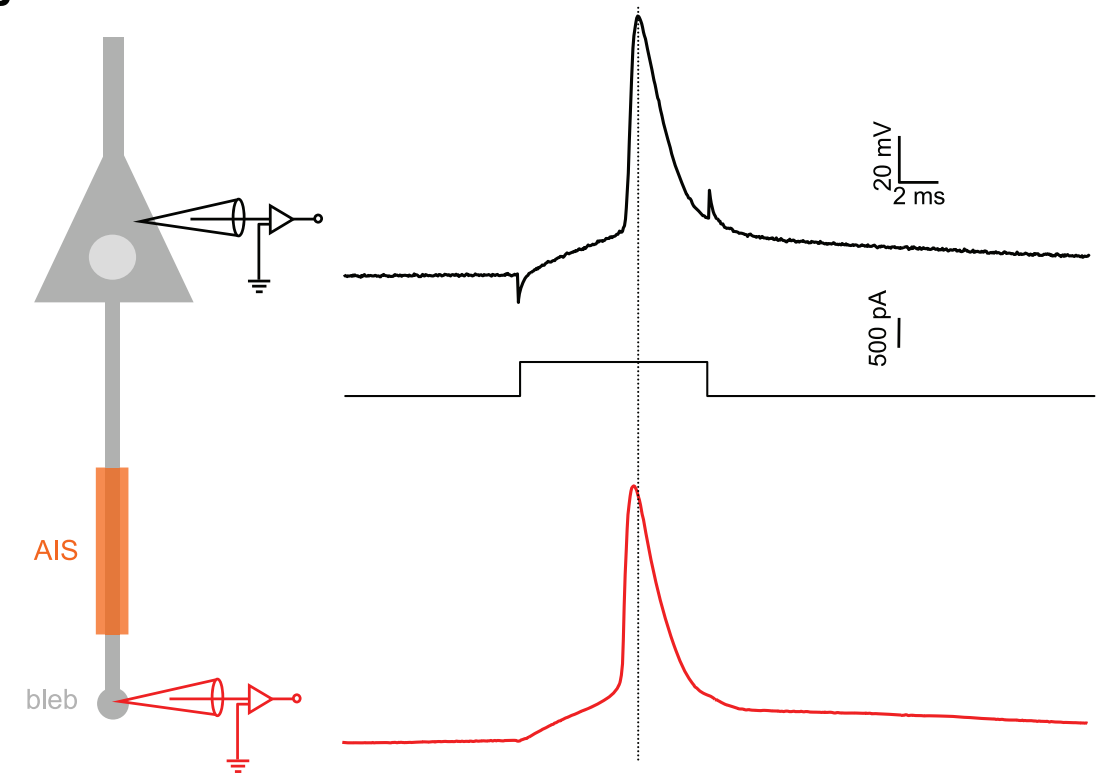

C

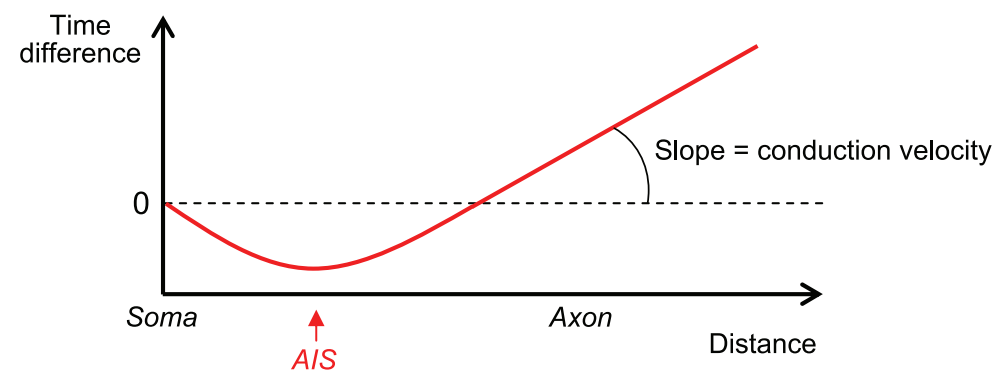

FIG. 5. Spike initiation in the AIS. A: confocal images of two L5 pyramidal neurons labeled with biocytin (A. Bialowas, P. Giraud, and D. Debanne, unpublished data). Note the characteristic bulbous end of the severed axon ("bleb") $B$ : dual soma-axonal bleb recording in whole-cell configuration from a L5 pyramidal neuron. Left: scheme of the recording configuration. Right: action potentials measured in the soma (black) and in the axon (red). $C$ : determination of the spike initiation zone. Scheme of the time difference between axonal and somatic spikes as a function of the axonal distance (origin: soma). The maximal advance of the axonal spike is obtained at the AIS (i.e., the spike initiation zone). The slope of the linear segment of the plot gives an estimate of the conduction velocity along the axon.
15-20 $\mu \mathrm{m}$ from the soma; Ref. 284). Here, the authors found that the AIS but not the first NoR was very highly sensitive to focal application of a low concentration of TTX. Initiation in the AIS has recently been confirmed by the use of noninvasive recording techniques $(196,396)$. The origin of the discrepancy between the first two studies has been elucidated. In fact, cell-attached recordings from the axon initial segment are not appropriate because the capacitive and ionic currents overlap, preventing identification of the spike onset.

In unmyelinated axons, the initiation zone has been identified at 20-40 $\mu \mathrm{m}$ from the axon hillock. In CA3 pyramidal neurons, the AP initiation zone is located at 35-40 $\mu \mathrm{m}$ from the soma (363). A much shorter distance 
has been reported in hippocampal granule cell axons. The site of initiation has been estimated at $20 \mu \mathrm{m}$ from the axon hillock (463). This proximal location of the spike initiation zone is corroborated by the labeling of sodium channels and ankyrin-G within the first $20 \mu \mathrm{m}$ of the axon (299). A possible explanation for this very proximal location might be that the very small diameter of granule cell axons ( $\sim 0.3 \mu \mathrm{m}$, Ref. 208) increases the electrotonic distance between the soma and proximal axonal compartments, thus isolating the site of initiation from the soma.

\section{Threshold of action potential initiation}

An essential feature of the all-or-none property of the action potential is the notion of a threshold for eliciting a spike. Converging evidence points to the fact that neuronal firing threshold may not be defined by a single value. The first studies of Lapicque (317) were designed to describe the role of depolarization time on the threshold current: the threshold current was reduced when its duration increased. Based on Hodgkin-Huxley membrane equations, Noble and Stein $(384,385)$ defined the spike threshold as the voltage where the summed inward membrane current exceeds the outward current.

In contrast with current threshold, voltage threshold could not be assessed in neurons until intracellular records were obtained from individual neurons (77). Given the complex geometry of the neuron, a major question was raised in the 1950s: is action potential threshold uniform over the neuron? Since the spike is initiated in the axon, it was postulated that the voltage threshold was $10-20 \mathrm{mV}$ lower (more hyperpolarized) in the AIS than in the cell body (124). Because direct recording from the axon was not accessible for a long time, there was little evidence for or against this notion. In an elegant study, Maarten Kole and Greg Stuart recently solved this question with direct patch-clamp recordings from the AIS (292). They showed that the current threshold to elicit an action potential is clearly lower in the AIS (Fig. 6A). However, the voltage threshold defined as the membrane potential at which the rate of voltage (i.e., the first deriv-
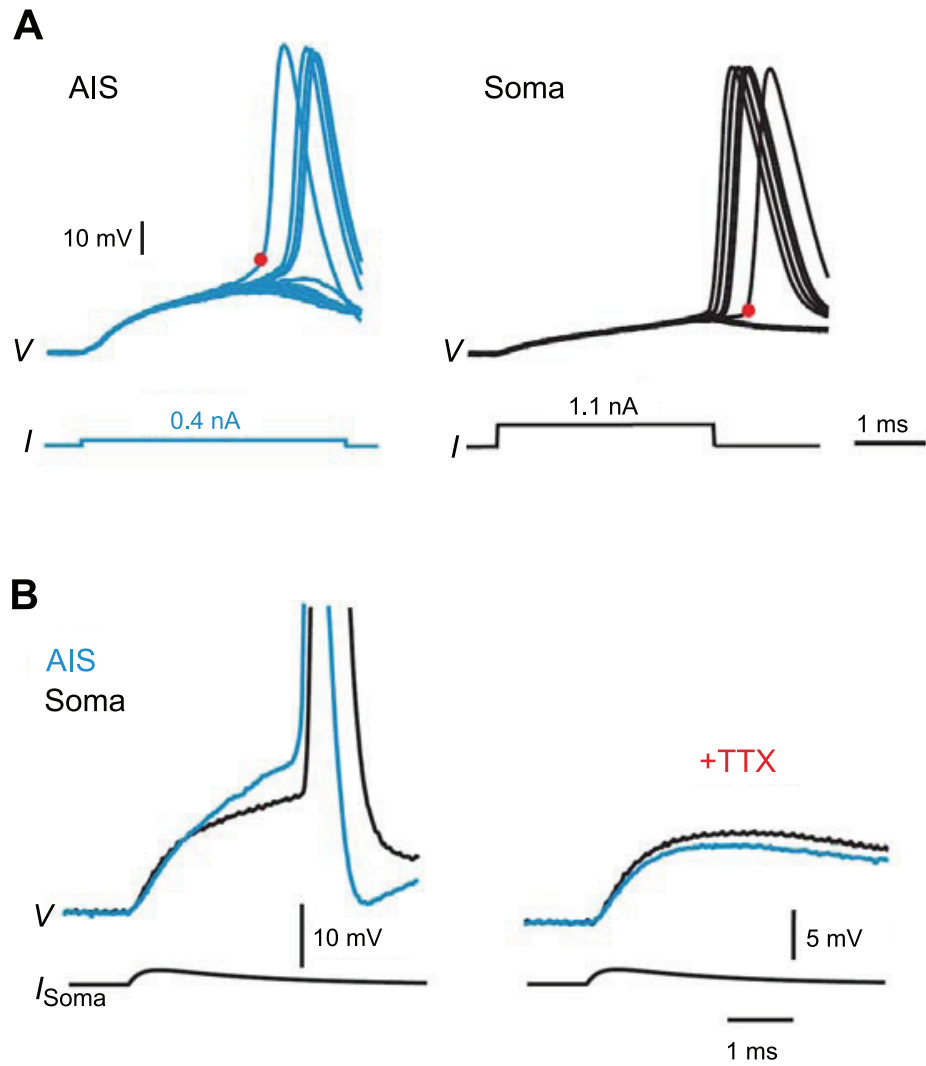

FIG. 6. Spike threshold is lowest in the AIS. A: lower currrent threshold but high voltage in the AIS of L5 pyramidal neurons. Left: overlaid voltage responses during current injection into the AIS (blue) or soma (black) at the action potential threshold. Note the depolarized voltage threshold in the AIS compared with the soma. Right: average amplitude of injected current versus action potential probability for action potentials evoked by current injection in the AIS (open circles) or soma (solid circles). Note the lower current threshold in the AIS. B: slow depolarizing ramp mediated by $\mathrm{Na}^{+}$channel in the AIS but not in the soma. Left: action potentials generated by simulated EPSC injection at the soma and recorded simultaneously at the soma (AIS) and AIS (blue). Middle: same recording in the presence of TTX (1 $\mu$ M). Right: voltage difference (AIS-soma) in control (gray) and TTX (red) reveals a depolarizing ramp in the AIS before spike intiation. [Adapted from Kole and Stuart (292), with permission from Nature Publishing Group.] 
ative) crosses a certain value (generally 10-50 V/s, Refs. 10, 201, 471) appeared surprisingly to be highest in the axon (Fig. 6A). This counterintuitive observation is due to the fact that $\mathrm{Na}^{+}$channels in the AIS drive a local depolarizing ramp just before action potential initiation that attenuates over very short distances as it propagates to the soma or the axon proper, thus giving the impression that the voltage threshold is higher (Fig. 6B). When this local potential is abolished by focal application of TTX to the AIS, then the voltage threshold is effectively lower in the AIS (292). In other words, the spike threshold measured out of the AIS is a propagating spike, and the correct measure in this compartment is the threshold of the SD component. This subtlety may also be at the origin of unconventional proposals for $\mathrm{Na}^{+}$channel gating during action potential initiation (35, 236, 359, 379, 578). Indeed, the onset of the action potential appears faster in the soma than expected from Hodgkin-Huxley modelling.

The spike threshold is not a fixed point but rather corresponds to a range of voltage. For instance, intrasomatic recordings from neocortical neurons in vivo reveal that spike threshold is highly variable $(26,27,248)$. The first explanation usually given to account for this behavior involves channel noise. In fact, the generation of an AP near the threshold follows probability laws because the opening of voltage-gated channels that underlie the sodium spike is a stochastic process $(465,560)$. The number of voltage-gated channels is not large enough to allow the contribution of channel noise to be neglected.

However, this view is now challenged by recent findings indicating that the large spike threshold variability measured in the soma results from back-propagation of the AP from the AIS to the soma when the neuron is excited by trains of noisy inputs (578). In fact, at the point of spike initiation (i.e., the AIS), the spike is generated with relatively low variance in membrane potential threshold but as it back-propagates towards the soma, variability increases. This behavior is independent of channel noise since it can be reproduced by a deterministic Hodgkin-Huxley model (578). The apparent increase in spike threshold variance results in fact from the rearrangement of the timing relationship between spikes and the frequency component of subthreshold waveform during propagation.

\section{Timing of action potential initiation}

Synchronous population activity is critical for a wide range of functions across many different brain regions including sensory processing (491), spatial navigation (388), and synaptic plasticity $(53,142,143)$. Whereas temporal organization of network activity clearly relies on both timing at the synapse (70) and elsewhere within the network (441), the mechanisms governing precise spike timing in individual neurons are determined at the AIS.
Recently, the rules governing the temporal precision of spike timing have started to emerge.

Outward voltage-gated currents with biophysical properties that sharpen physiological depolarizations, such as EPSPs, reduce the time window during which an action potential can be triggered and thus enhance spike precision $(25,200,207)$. In contrast, outward currents that reduce the rate of depolarization leading to the generation of a spike decrease spike-time precision (131, 503). Here, high spike jitter may result from the fact that channel noise near the threshold becomes determinant during slow voltage trajectories. With the recent development of axonal recordings, it will be important to determine how these currents shape voltage in the AIS.

\section{Plasticity of action potential initiation}

The probability of action potential initiation in response to a given stimulus is not absolutely fixed during the life of a neuron but subjected to activity-dependent regulation. In their original description of LTP, Bliss and Lømo (63) noticed that the observed increase in the population spike amplitude, which reflects the number of postsynaptic neurons firing in response to a given synaptic stimulation, was actually greater than simply expected by the LTP-evoked increase in the population EPSP (63). This phenomenon was termed EPSP-spike or E-S potentiation. The intracellular signature of E-S potentiation is an increased probability of firing in response to a given synaptic input. This plasticity appears to be of fundamental importance because it directly affects the input-output function of the neuron. Originally described in the dentate gyrus of the hippocampus, E-S potentiation was also found at the Schaffer collateral-CA1 cell synapse when the afferent fibers were tetanized $(1,9,136)$ and maybe induced associatively with coincident activation of synaptic input and a back-propagated action potential (91). Although dendritic conductances such as A-type $\mathrm{K}^{+}$(199) or h-type currents (90) are implicated in its expression, regulation of axonal channels cannot totally be excluded. Indeed, hyperpolarization of the spike threshold has been encountered in many forms of long-lasting increase in excitability in cerebellar and hippocampal neurons (3, 568). Furthermore, activation of the fast transient $\mathrm{Na}^{+}$ current is regulated following LTP induction in CA1 pyramidal neurons (568).

\section{B. Conduction of Action Potentials Along the Axon}

\section{A brief overview of the principle of conduction in unmyelinated axons}

Conduction of the action potential has been primarily studied and characterized in invertebrate axons. According to a regenerative scheme, propagation along unmyeli- 
nated axons depends on the passive spread of current ahead of the active region to depolarize the next segment of membrane to threshold. The nature of the current flow involved in spike propagation is generally illustrated by an instantaneous picture of the action potential plotted spatially along the axon. Near the leading edge of the action potential there is a rapid influx of sodium ions that will lead to depolarization of a new segment of membrane towards threshold. At the following edge of the action potential, current flows out because potassium channels are opened, thus restoring the membrane potential towards the resting value. Because of both the inactivation of voltage-gated $\mathrm{Na}^{+}$channels and the high conductance state of hyperpolarizing $\mathrm{K}^{+}$channels, the piece of axonal membrane that has been excited is not immediately reexcitable. Thus the action potential cannot propagate backward, and conduction is therefore generally unidirectional. As the action potential leaves the activated region, $\mathrm{Na}^{+}$channels recover from inactivation, $\mathrm{K}^{+}$conductance declines, and the membrane is thus susceptible to be reexcited.

\section{Conduction in myelinated axons}

In myelinated (or medulated) axons, conduction is saltatory (from Latin saltare, to jump). Myelin is formed by wrapped sheaths of membrane from Schwann cells in peripheral nerves and oligodendrocytes in central axons. The number of wrappings varies between 10 and 160 (17). The presence of the myelin sheath has a critical impact on the physiology of the axon. The effective membrane resistance of the axon is locally increased by several orders of magnitude (up to 300 times), and the membrane capacitance is reduced by a similar factor. The myelin sheath is interrupted periodically by NoR, exposing patches of axonal membrane to the external medium. The internodal distance is usually 100 times the external diameter of the axon, ranging between $200 \mu \mathrm{m}$ and $2 \mathrm{~mm}(264,453)$. The electrical isolation of the axon by myelin restricts current flow to the node, as ions cannot flow into or out of the high-resistance internodal region. Thus only the restricted region of the axon at the node is involved in impulse propagation. Therefore, the impulse jumps from node to node, thereby greatly increasing conduction velocity. Another physiologically interesting consequence of myelination is that less metabolic energy is required to maintain the gradient of sodium and potassium since the flow of these ions is restricted to the nodes. However, a recent study from the group of Geiger (7) indicates that because of matched properties of $\mathrm{Na}^{+}$and $\mathrm{K}^{+}$channels, energy consumption is minimal in unmyelinated axons of the hippocampus.

The principle of saltatory conduction was first suggested by Lillie in 1925 (326) and later confirmed by direct experimental evidence $(69,265,407,527)$. In their seminal paper, Huxley and Stämpfli (265) measured currents in electrically isolated compartments of a single axon, containing either a node or an internode, during the passage of an impulse. They noticed that when the compartment contained a NoR, stimulation of the nerve resulted in a large inward current. In contrast, no inward current was recorded when the chamber contained an internode, thus indicating that there is no regenerative activity. The discontinuous nature of saltatory conduction should not be emphasized too much, however, because 30 consecutive NoR can participate simultaneously in some phases of the action potential.

\section{Conduction velocity}

Conduction velocity in unmyelinated axons depends on several biophysical factors such as the number of available $\mathrm{Na}^{+}$channels, membrane capacitance, internal impedance, and temperature (122, 148, 251, 252, 277). Conduction velocity can be diminished by reducing external $\mathrm{Na}^{+}$concentration (277) or partially blocking $\mathrm{Na}^{+}$ channels with a low concentration of TTX (122). In fact, the larger the sodium current, the steeper the rate of rise of the action potential. As a consequence, the spatial voltage gradient along the fiber is steeper, excitation of adjacent axonal regions is faster, and conduction velocity is increased.

The second major determinant of conduction is membrane capacity. Membrane capacity determines the amount of charge stored on the membrane per unit area. Thus the time necessary to reach the threshold is obviously shorter if the capacity is small.

The third major parameter for conduction velocity is the resistance of the axoplasm (i.e., the intra-axonal medium). For instance, in the giant squid axon, the insertion of a low-impedance wire cable in the axon considerably increases the rate of conduction (148). This property explains why conduction velocity in unmyelinated axons is proportional to the square root of the axon diameter (251). In fact, the current flow is facilitated in largediameter axons because of the high intracellular ion mobility.

Temperature has large effects on the rate of increase of $\mathrm{Na}^{+}$channel conductance and action potential waveform (253). Channels open and close more slowly at lower temperature, and subsequently conduction velocity is reduced $(106,198)$.

In myelinated axons, conduction displays linear dependence on fiber diameter $(17,264,444,453)$. A simple rule is that every micrometer of outer diameter adds $6 \mathrm{~m} / \mathrm{s}$ to the conduction velocity at $37^{\circ} \mathrm{C}$. One particularly fascinating point addressed by the theoretical work of Rushton (453) is the notion of invariance in the conduction properties and morphological parameters of myelinated axons. In fact, the geometry of myelinated axons 
seems to be tuned by evolution to give the highest conduction velocity.

Conduction velocity in mammalian axons has been evaluated traditionally by antidromic latency measurements or field measurements of axon volleys $(300,521)$. More direct measurements of conduction velocity have been obtained recently with the development of axonal patch-clamp recordings in brain tissue. In unmyelinated axons, conduction velocity is generally slow. It has been estimated to be close to $0.25 \mathrm{~m} / \mathrm{s}$ at Schaffer collateral (8) or at the mossy-fiber axon $(299,463)$, and reach $0.38 \mathrm{~m} / \mathrm{s}$ in the axon of CA3 pyramidal neurons (363). In contrast, conduction becomes faster in myelinated axons, but it largely depends on the axon diameter. In fact, myelination pays in terms of conduction velocity when the axon diameter exceeds 1-2 $\mu \mathrm{m}$ (453). In the thin Purkinje cell axon $(\sim 0.5-1 \mu \mathrm{m})$, conduction velocity indeed remains relatively slow $(0.77 \mathrm{~m} / \mathrm{s}$; Ref. 116$)$. Similarly, in the myelinated axon of L5 neocortical pyramidal neurons of the rat (diameter $\sim 1-1.5 \mu \mathrm{m}$; Ref/ 290), conduction velocity has been estimated to be $2.9 \mathrm{~m} / \mathrm{s}$ (291). Conduction velocity along small axons of neurons from the subthalamic nucleus is also relatively modest $(4.9 \mathrm{~m} / \mathrm{s}$; diameter $\sim 0.5$ $\mu \mathrm{m}$; Ref. 22). In contrast, in large-diameter axons such as cat brain stem motoneuron fibers $(\sim 5 \mu \mathrm{m})$, the conduction velocity reaches $70-80 \mathrm{~m} / \mathrm{s}$ (214). Similarly, in group I afferents of the cat spinal cord, conduction velocity has been estimated to vary between 70 and $90 \mathrm{~m} / \mathrm{s}$ (309). The fastest impulse conduction in the animal kingdom has been reported in myelinated axons of the shrimp, which are able to conduct impulses at speeds faster than $200 \mathrm{~m} / \mathrm{s}$ (569). These axons possess two unique structures (microtubular sheath and submyelinic space) that contribute to speed-up propagation. In particular, the submyelinic space constitutes a low-impedance axial path that acts in a similar way to the wire in the experiment of del Castillo and Moore (148).

\section{Modulation of conduction velocity}

Conduction velocity along myelinated axons has been shown to depend also on neuron-glia interactions $(123,190,526,570)$. Importantly, depolarization of a single oligodendrocyte was found to increase the action potential conduction velocity of the axons it myelinates by $\sim 10 \%$ (570). Although the precise mechanism has not been yet fully elucidated, it may result from ephaptic interaction between the myelin depolarization and the axon (280; see also sect. VIIIC). This finding may have important functional consequences. Mature oligodendrocytes in the rat hippocampus are depolarized by theta burst stimulation of axons. Thus myelin may also dynamically regulate impulse transmission through axons and promote synchrony among the multiple axons under the domain of an individual oligodendrocyte (570). In a recent study, the conduction velocity in small myelinated axons was found to depend on tight junctions between myelin lamellae (153). The absence of these tight junctions in Claudin 11-null mice does not perturb myelin formation but significantly decreases conduction velocity in small, but not in large, myelinated axons. In fact, tight junctions in myelin potentiate the insulation of small axons, which possess only a relatively limited number of myelin loops, by increasing their internodal resistance.

In auditory thalamocortical axons, nicotine enhances conduction velocity and decreases axonal conduction variability (282). Although the precise mechanism remains to be clarified, this process may lower the threshold for auditory perception by acting on the thalamocortical flow of information.

\section{FUNCTIONAL COMPUTATION IN THE AXON}

\section{A. Activity-Dependent Shaping of the Presynaptic Action Potential}

The shape of the presynaptic action potential is of fundamental importance in determining the strength of synapses by modulating transmitter release. The waveform of the depolarization dictates the calcium signal available to trigger vesicle fusion by controlling the opening of voltage-gated calcium channels and the driving force for calcium influx. Two types of modification of the presynaptic action potential have been reported experimentally: modifications of action potential width and/or modifications of action potential amplitude.

\section{Activity-dependent broadening of presynaptic action potential}

The duration of the presynaptic spike is not fixed, and activity-dependent short-term broadening of the spike has been observed in en passant mossy fiber boutons (209). The mossy fiber-CA3 pyramidal cell synapse displays fast synchronized transmitter release from several active zones and also shows dynamic changes in synaptic strength over a more than 10-fold range. The exceptionally large synaptic facilitation is in clear contrast to the weak facilitation $(\sim 150 \%$ of the control) generally observed at most central synapses. Granule cell axons exhibit several voltage-gated potassium channels including Kv1.1 (443), Kv1.2 (477), and two A-type potassium channels, Kv1.4 (126, 478, 543) and Kv3.4 (543). Geiger and Jonas (209) have shown that the action potential at the mossy fiber terminal is half as wide as that at the soma. During repetitive stimulation, the action potential gets broader in the axon terminal but not in the soma (209) (Fig. 7). More interestingly, using simultaneous recordings from the granule cell terminal and the corre- 
A

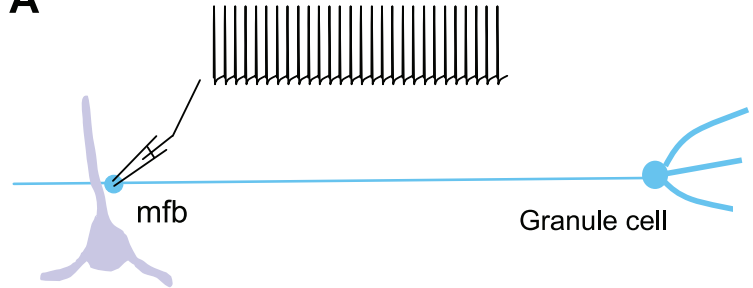

B

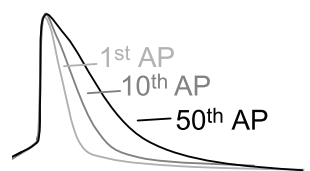

C

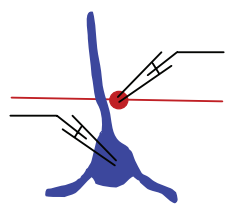

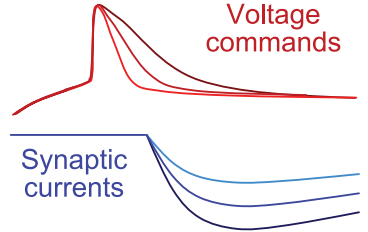

FIG. 7. Shaping of the action potential in the axon. A: a mossy fiber bouton (mfb, blue) is recorded in the whole cell configuration and activated

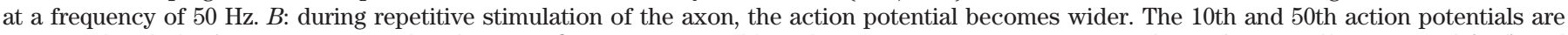

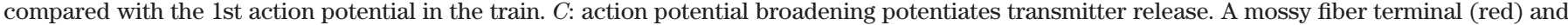

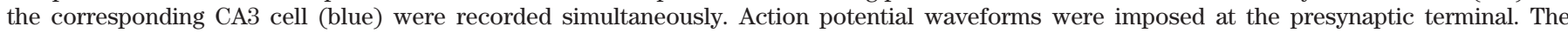

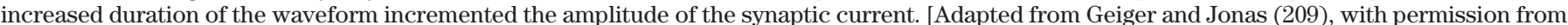
Elsevier.]

sponding postsynaptic apical dendrite of a CA3 neuron, Geiger and Jonas (209) showed that action potential broadening enhanced presynaptic calcium influx and doubled the EPSC amplitude (Fig. 7). This broadening results from the inactivation of A-type $\mathrm{K}^{+}$channels located in the membrane of the terminal. Consequently, the pronounced short-term facilitation probably results from the conjugated action of spike widening and the classical accumulation of residual calcium in the presynaptic terminal. Because ultrastructural analysis reveals A-type channel immunoreactivity in the terminal but also in the axonal membrane (126), activity-dependent spike broadening might also occur in the axon.

\section{Activity-dependent reduction of presynaptic action potential}

Reduction of the amplitude of the presynaptic action potential has been reported following repetitive stimulation of invertebrate (230) or mammalian axons $(209,552)$. This decline results from sodium channel inactivation and can be amplified by low concentrations of TTX $(78,343)$. The consequences of sodium channel inactivation on synaptic transmission have been studied at various central synapses. Interestingly, the reduction of the sodium current by application of TTX in the nanomolar range decreases glutamatergic transmission and enhances shortterm depression $(78,243,421)$. In addition, the depolarization of the presynaptic terminal by raising the external potassium concentration increases paired-pulse synaptic depression at autaptic contacts of cultured hippocampal cells (243) and decreases paired-pulse synaptic facilitation at Schaffer collateral-CA1 synapses stimulated extracellularly (364). In this case, the depolarization of the presynaptic axons is likely to enhance presynaptic spike attenuation. Importantly, inactivation of sodium channels by high external potassium increases the proportion of conduction failures during repetitive extracellular stimulation of Schaffer collateral axons (364). However, these results must be interpreted carefully because apparent changes in paired-pulse ratio may simply be the result of stimulation failures produced by the reduction in presynaptic axon excitability.

Interestingly, the manipulations of the sodium current mentioned above have little or no effect on GABAergic axons (243, 364, 421). Riluzole, TTX, or external potassium affect neither GABAergic synaptic transmission nor short-term GABAergic plasticity. This difference between glutamatergic and GABAergic axons might result from several factors. Sodium currents in interneurons are less sensitive to inactivation, and a slow recovery from inactivation has been observed for pyramidal cells but not for inhibitory interneurons (353). Moreover, the density of sodium current is higher in interneurons than in pyramidal neurons (354). Thus axons of GABAergic interneurons could be better cables for propagation than those of pyramidal cells $(194,525)$. This unusual property could be important functionally: safe propagation along inhibitory axons could protect the brain from sporadic hyperactivity and prevent the development of epileptiform activity.

\section{B. Signal Amplification Along the Axon}

Signal amplification is classically considered to be achieved by the dendritic membrane, the cell body, or the proximal part of the axon $(21,512)$. Whereas action potential propagation along the axon is clearly an active process that depends on a high density of sodium channels, the process of action potential invasion into presynaptic terminals was, until recently, less well understood. This question is of primary importance because the geometrical perturbation introduced by the presynaptic terminal decreases the safety factor for action potential propagation and may affect the conduction time (see sect. VIII). The invasion of the spike is active at the amphibian neuromuscular junction (278) but passive at the neuromuscular junction of the mouse $(76,163)$ and the lizard (329). This question has been reconsidered at hippocampal mossy fiber boutons (179). In this study, Engel and Jonas (179) showed that sodium channel density is very high at the presynaptic terminal (2,000 channels/mossy 
fiber bouton). In addition, sodium channels in mossy fiber boutons activate and inactivate with submillisecond kinetics. A realistic computer simulation indicates that the density of sodium channels found in the mossy fiber bouton not only amplifies the action potential but also slightly increases the conduction speed along the axon (179). Similarly, presynaptic sodium channels control the resting membrane potential at the presynaptic terminal of the calyx of Held (260), and hence may determine transmitter release at this synapse.

Another mechanism of activity-dependent signal amplification has been reported at hippocampal mossy fiber (376). In immature hippocampus, repetitive stimulation of the mossy fiber pathway not only facilitates synaptic transmission but also facilitates the amplitude of the presynaptic volley, the electrophysiological signature of the presynaptic action potential in the axon recorded extracellularly. This axonal facilitation is not observed in mature hippocampus. It is associated with the depolarization of mossy fibers and fully inhibited by $\mathrm{GABA}_{\mathrm{A}}$ receptor antagonists, indicating that GABA released from interneurons depolarizes the axon and increases its excitability. Because the presynaptic axon was not directly recorded in this study, further investigations will be necessary to determine whether $\mathrm{GABA}_{\mathrm{A}}$ receptor depolarization limits conduction failures or interacts with sodium channel amplification.

\section{Axonal Integration (Analog Signaling)}

Classically, the somatodendritic compartment is considered as the locus of neuronal integration where subthreshold electrical signals originating from active synapses are temporally summated to control the production of an output message, the action potential. According to this view, the axon initial segment is the final site of synaptic integration, and the axon remains purely devoted to action potential conduction in a digital way. Synaptic strength can be modulated by the frequency of presynaptic action potential firing. Today, this view is now challenged by accumulating evidence in invertebrate and vertebrate neurons showing that the axon is also able to integrate electrical signals arising from the somato-dendritic compartment of the neuron (for reviews, see Refs. $4,115,351,410)$. In fact, the axon appears now to be a hybrid device that transmits neuronal information through both action potentials in a digital way and subthreshold voltage in an analog mode.

\section{Changes in presynaptic voltage affect synaptic efficacy}

The story started with classical observations reported at the neuromuscular junction of the rat $(261,262)$, and at the giant synapse of the squid (237, 369, 524), where the membrane potential of the presynaptic axon was found to control the efficacy of action potentialtriggered synaptic transmission. Synaptic transmission was found to be gradually enhanced when the membrane potential of the presynaptic element was continuously hyperpolarized to different membrane potentials. Thus the membrane potential of the presynaptic element determines, in an analog manner, the efficacy of the digital output message (the action potential). This facilitation was associated with a reduction in the paired-pulse ratio (369), indicating that it results from enhanced presynaptic transmitter release. Although the mechanisms underlying this behavior have not been clearly identified, it should be noted that graded presynaptic hyperpolarization increased the presynaptic spike amplitude in a graded manner $(237,369,524)$. The importance of the amplitude of the presynaptic action potential is also demonstrated by the reduction of the evoked EPSP upon intracellular injection of increasing concentrations of TTX in the presynaptic axon (279). Thus a possible scheme here would be that hyperpolarization of the presynaptic element induces $\mathrm{Na}^{+}$channel recovery from inactivation and subsequently enhance presynaptic spike and EPSP amplitudes. A similar phenomenon has been recently observed at autaptic contacts in cultured hippocampal neurons (528).

A totally different scenario has been observed in the Aplysia (475, 486, 487) and the leech (382). In these studies on connected pairs of neurons, the authors reported that constant or transient depolarization of the membrane potential in the soma of the presynaptic neuron facilitates synaptic transmission evoked by single action potentials, in a graded manner (Fig. 8A). The underlying mechanism in Aplysia neurons involves the activation of steady-state $\mathrm{Ca}^{2+}$ currents (475) and inactivation of 4-aminopyridine-sensitive $\mathrm{K}^{+}$current $(484,485)$ which overcome propagation failures in a weakly excitable region of the neuron (184). Thus the possible scenario in Aplysia neurons is that somatic depolarization may inactivate voltage-gated $\mathrm{K}^{+}$currents located in the axon that control the propagation and subsequently the amplitude and duration of the action potential.

It is also important to mention that many types of invertebrate neuron release neurotransmitter as a graded function of presynaptic membrane potential (11, 86, 227). In these examples, synaptic transmission does not strictly depend on spiking but rather on variations of the presynaptic membrane potential, further supporting the idea that membrane potential alone is capable of controlling neuronal communication in an analog manner.

\section{Space constant in axons}

In the experiments reported in Aplysia, facilitation was induced by changing membrane potential in the soma, indicating that the presynaptic terminal and the cell 


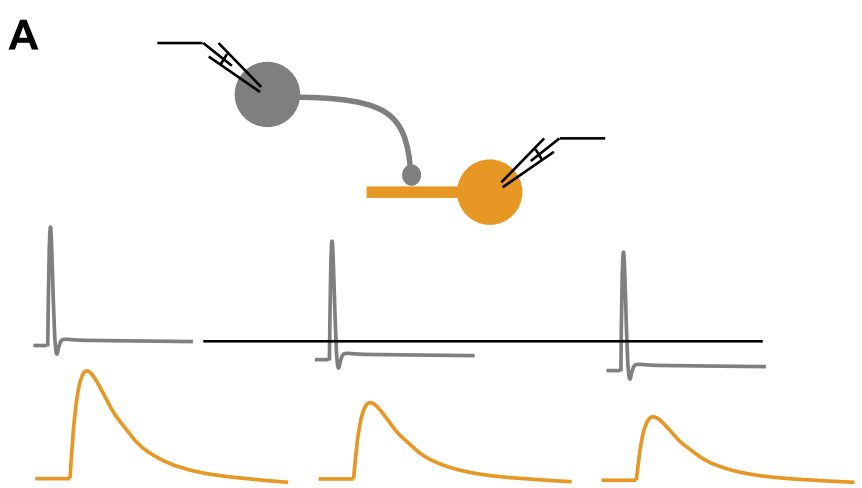

B
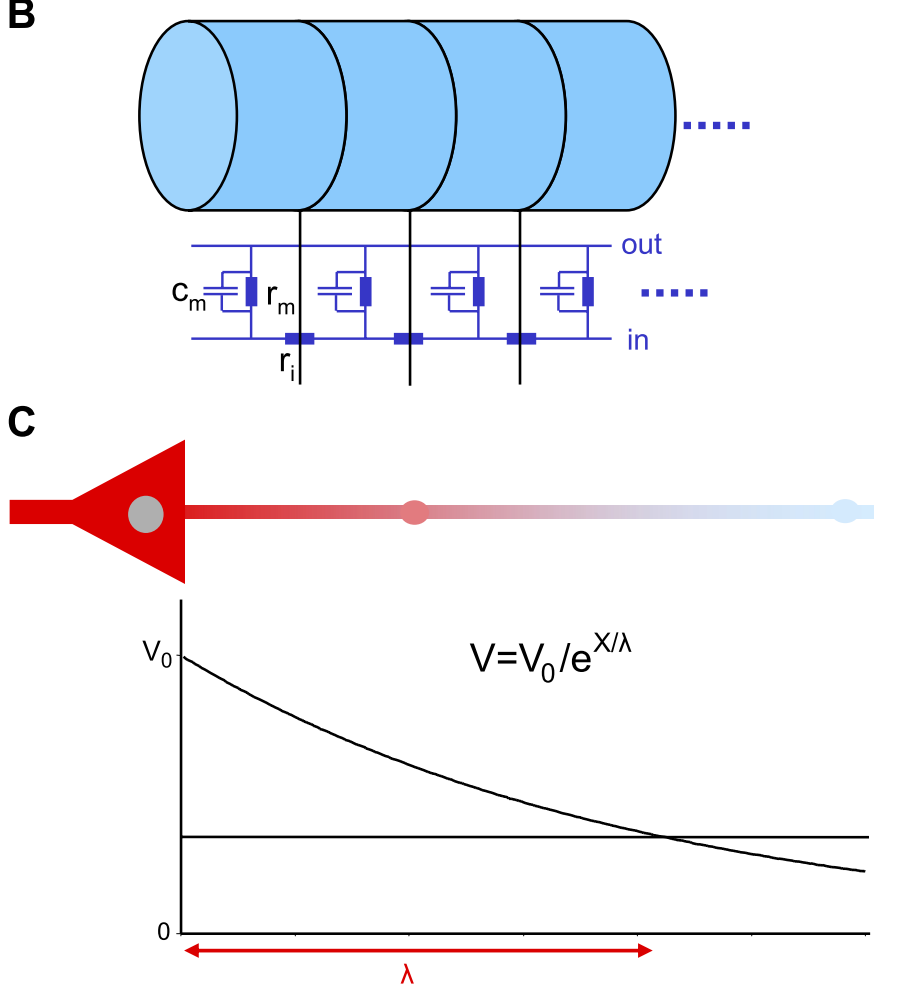

FIG. 8. Axonal integration. $A$ : graded control of synaptic efficacy by the membrane potential in a pair of connected Aplysia neurons. The hyperpolarization of the presynaptic neuron gradually reduces the amplitude of the synaptic potential. [Adapted from Shimahara and Tauc (487).] B: electrical model of a passive axon. Top: the axon is viewed as a cylinder that is subdivided into unit lengths. Bottom: each unit length is considered as a parallel circuit with its membrane resistance $\left(r_{\mathrm{m}}\right)$ and capacitance $\left(c_{\mathrm{m}}\right)$. All circuits are connected intracellularly by resistors $\left(r_{\mathrm{i}}\right) . C$ : space constant of the axon. Top: schematic representation of a pyramidal cell with its axon. Bottom: plot of the voltage along the axon A depolarization to $V_{0}$ is applied at the cell body (origin in the plot). The potential decays exponentially along the axon according to $V=V_{0} / e^{\mathrm{X} / \lambda}$. The color represents the membrane potential (red is depolarized and blue is the resting potential). The space constant $\lambda$ is defined as the distance for which $V$ is $37 \%$ of $V_{0}$ (dashed horizontal line on the plot).

body are not electrically isolated. Thus the biophysical characteristics of electrical transfer along the axon appear as the critical parameter determining axonal integration of somatic signals.

For biophysicists, the axon is viewed as a cylinder that can be subdivided into unit lengths. Each unit length is a parallel circuit with its own membrane resistance $\left(r_{m}\right)$ and capacitance $\left(c_{\mathrm{m}}\right)$. All the circuits are connected by resistors $\left(r_{\mathrm{i}}\right)$, which represent the axial resistance of the intracellular cytoplasm, and a short circuit, which represents the extracellular fluid (Fig. $8 B$ ). The voltage response in such a passive cable decays exponentially with distance due to electrotonic conduction (253a). The space (or length) constant, $\lambda$, of axons is defined as the distance over which a voltage change imposed at one site will drop to $1 / e(37 \%)$ of its initial value (Fig. $8 C$ ). In fact, the depolarization at a given distance $x$ from the site of injection $x=0$ is given by $V_{x}=V_{0} / e^{x / \lambda}$, where $e$ is the Euler's number and $\lambda$ is the space or length constant. The length constant is expressed as $\lambda=\left(r_{\mathrm{m}} / r_{\mathrm{i}}\right)^{1 / 2}$. For a cable with a diameter $d$, it is therefore expressed as $\lambda=[(d /$ 4) $\left.\left(R_{\mathrm{M}} / R_{\mathrm{A}}\right)\right]^{1 / 2}$, where $R_{\mathrm{A}}$ is the axial resistance and $R_{\mathrm{M}}$ is the specific membrane resistance (425). Thus the length constant of the axon depends on three main parameters. In myelinated axons, $R_{\mathrm{M}}$ may reach very high values because of the myelin sheath. Therefore, space constants in myelinated axons are very long. For instance, in cat brain stem neurons, the space constant amounts to 1.7 $\mathrm{mm}$ (214). EPSPs generated in the soma are thus detectable at long distances in the axon. In thin unmyelinated axons, the situation was thought to be radically different because $R_{\mathrm{M}}$ is relatively low and the diameter might be very small. Space constants below $200 \mu \mathrm{m}$ were considered in models of nonmyelinated axons $(295,313)$. The recent use of whole-cell recordings from unmyelinated axons (58) profoundly changed this view. In hippocampal granule cell axons, the membrane space constant for an EPSP generated in the somato-dendritic compartment is $\sim 450 \mu \mathrm{m}$ (5; see also sect. VC3). Similarly, the axonal space constant in L5 pyramidal neurons is also $450 \mu \mathrm{m}$ (489). However, these values might be underestimated because the EPSP is a transient event and the space constant is inversely proportional to the frequency content of the signal $(468,489)$. For instance, the axonal space constant for slow signals (duration $\geq 200 \mathrm{~ms}$ ) may reach $\sim 1,000 \mu \mathrm{m}$ in L5 pyramidal cell axons (112).

\section{Axonal integration in mammalian neurons}

Axonal integration is not peculiar to invertebrate neurons and synaptic facilitation, produced by depolarization of the presynaptic soma, has been reported in at least three central synapses of the mammalian brain. First, at synapses established between pairs of CA3-CA3 pyramidal cells, steady-state depolarization of the presynaptic neuron from -60 to $-50 \mathrm{mV}$ enhances synaptic transmission (460). More recently, an elegant study published by Alle and Geiger (5) shows, by using direct patch-clamp recording from presynaptic hippocampal mossy fiber boutons, that granule cell axons transmit analog signals (membrane potential at the cell body) in 
addition to action potentials. Surprisingly, excitatory synaptic potentials evoked by local stimulation of the molecular layer in the dentate gyrus could be detected in the mossy-fiber bouton located at several hundreds microns from the cell body (Fig. 9). Excitatory presynaptic potentials (EPreSP) recorded in the mossy-fiber bouton represent forward-propagated EPSPs from granule cell dendrite. They were not generated locally in the CA3 region because application of AMPA receptor or sodium channel blockers locally to CA3 has no effect on the amplitude of EPreSP (5).

As expected from cable theory, this signal is attenuated and the EPSP waveform is much slower in the terminal than in the soma of granule cells. The salient finding here is that the space constant of the axon is much wider $(\sim 450 \mu \mathrm{m})$ than initially expected. Consistent with propagation of electrical signals over very long distances, the analog facilitation of synaptic transmission has a slow time constant (2-4 s; Refs. 291, 382, 489). The functional consequence is that slow depolarizations of the membrane in somatic and dendritic regions are transmitted to the axon terminals and can influence the release of transmitter at the mossy fiber-CA3 cell synapse. Similar observations have been reported in the axon of L5 cortical
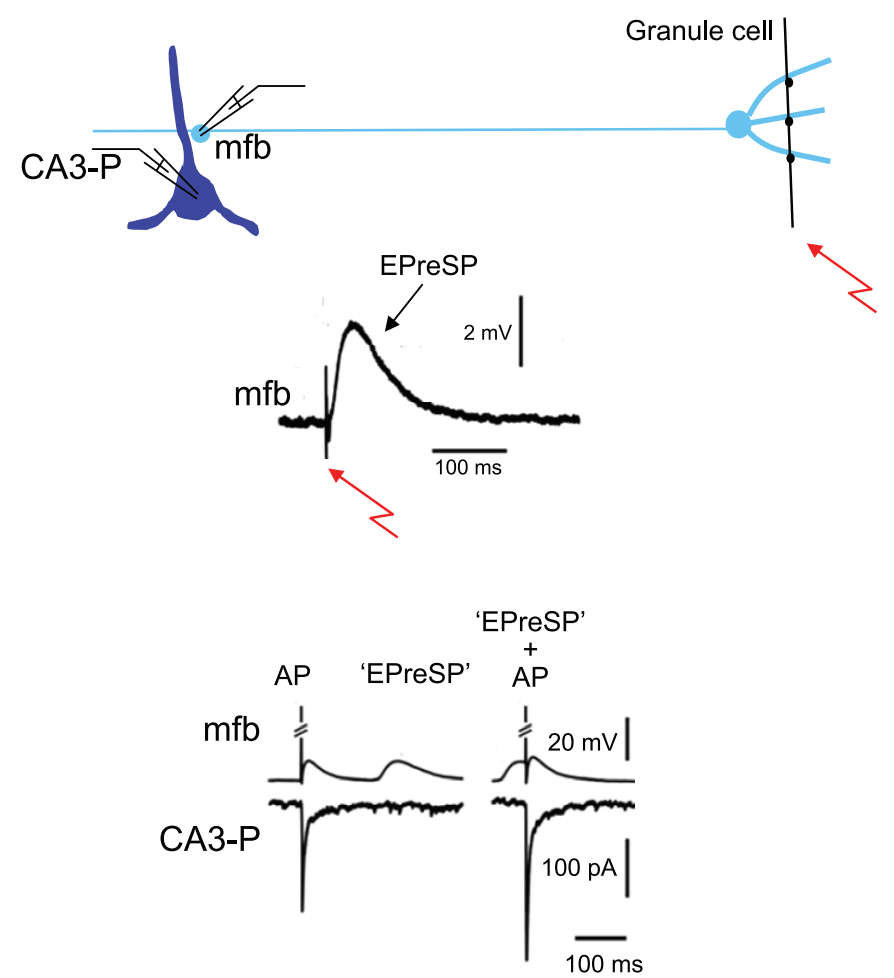

FIG. 9. Integration of subthreshold synaptic potential in the axon of hippocampal granule cells. Electrically evoked synaptic inputs in the dendrites of a granule cell can be detected in the mossy fiber terminal (EPreSP). Bottom panel: synaptic transmission at the mossy fiber synapse was facilitated when the simulated EPreSP ("EPreSP") was associated with a presynaptic action potential (AP + "EPreSP"). [Adapted from Alle and Geiger (5), with permission from the American Association for the Advancement of Science.] pyramidal neurons recorded in whole cell configuration at distances ranging between 90 and $400 \mu \mathrm{m}$ (489; Fig. 10A). In this case, whole cell recording is possible on the axon because sectioning the axon produces a small enlargement of its diameter that allows positioning of a patch pipette. Here again, incoming synaptic activity in the presynaptic neuron propagates down the axon and can modulate the efficacy of synaptic transmission. The modulation of synaptic efficacy by somatic potential is blocked at L5-L5 connections (489) or reduced by $\mathrm{Ca}^{2+}$ chelators at the mossy fiber input (5; but see Ref. 468), and may therefore result from the control of background calcium levels at the presynaptic terminal (24).

At least one mechanism controlling the voltage-dependent broadening of the axonal action potential has been recently identified in L5 pyramidal neurons (291, 490). Kv1 channels are expressed at high densities in the AIS (267), but they are also present in the axon proper. With cell-attached recordings from the axon at different distances from the cell body, Kole et al. (291) elegantly showed that Kv1 channel density increases 10-fold over the first $50 \mu \mathrm{m}$ of the AIS but remains at very high values in the axon proper $(\sim 5$-fold the somatic density). The axonal current mediated by Kv1 channels inactivates with a time constant in the second range $(291,489)$. Pharmacological blockade or voltage inactivation of Kv1 channels produce a distance-dependent broadening of the axonal spike, as well as an increase in synaptic strength at proximal axonal terminals (291). For instance, when the membrane potential is shifted from -80 to $-50 \mathrm{mV}$, the D-type current is reduced by half (291). Subsequently, the axonal spike is enlarged and transmitter release is enhanced (Fig. 10B). Thus Kv1 channels occupy a strategic position to integrate slow subthreshold signals generated in the dendrosomatic region and control the presynaptic action potential waveform to finely tune synaptic coupling in local cortical circuits.

\section{Axonal speeding}

The role of the axonal membrane compartment is also critical in synaptic integration. The group of Alain Marty (365) showed by cutting the axon of cerebellar interneurons with two-photon illumination that the axonal membrane speeds up the decay of synaptic potentials recorded in the somatic compartment of cerebellar interneurons. This effect results from passive membrane properties of the axonal compartment. In fact, the axonal compartment acts as a sink for fast synaptic currents. The capacitive charge is distributed towards the axonal membrane synaptic current, thus accelerating the EPSP decay beyond the speed defined by the membrane time constant of the neuron (usually $20 \mathrm{~ms}$ ). Functionally, axonal speeding has important consequences. EPSP decay is faster and, consequently, axonal speeding increases the tempo- 
A
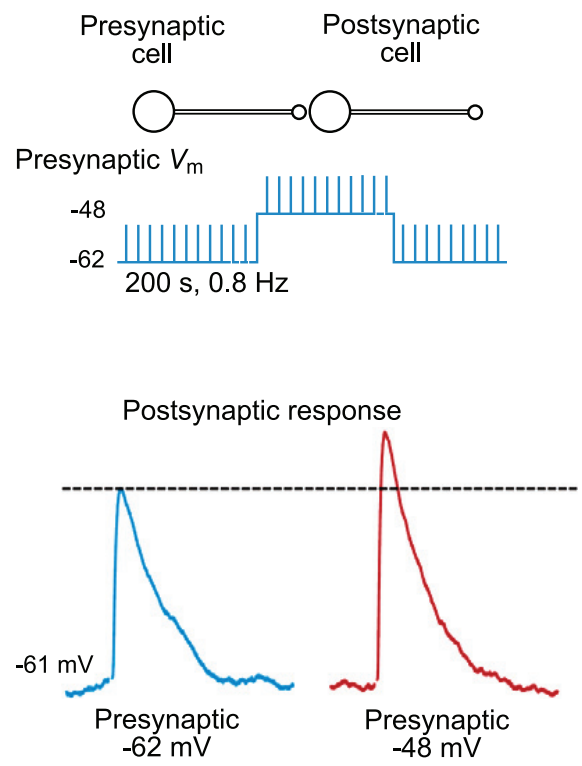

B
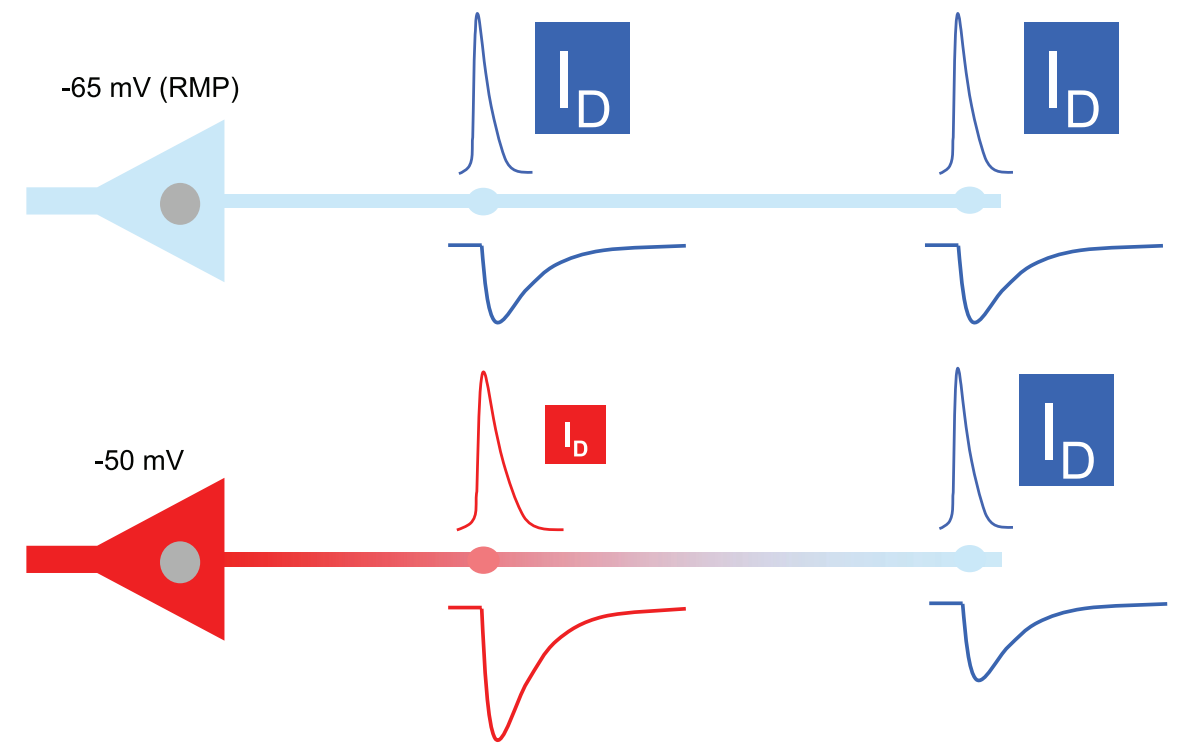

FIG. 10. Depolarization of the presynaptic soma facilitates synaptic transmission through axonal integration. A: facilitation of synaptic transmission in connected L5-L5 pyramidal neurons. Left: experimental design. Synaptic transmission is assessed when presynaptic action potentials are elicited either from rest $(-62 \mathrm{mV})$ or from a depolarized potential $(-48 \mathrm{mV})$. Right: averaged EPSP amplitude at two presynaptic somatic membrane potentials. Note the facilitation when the presynaptic potential is depolarized. [Adapted from Shu et al. (489), with permission from Naure Publishing Group.] B: mechanism of presynaptic voltage-dependent facilitation of synaptic transmission. Top: the cell body and the axon of a cortical pyramidal neuron are schematized. When an action potential is elicited from the resting membrane potential (RMP, $-65 \mathrm{mV})$, the spike in the axon is identical in the proximal and distal part of the axon. Postsynaptic inward currents are shown below. Bottom: an action potential elicited from a steady-state depolarized value of $-50 \mathrm{mV}$ is larger in the proximal part of the axon (because $I_{\mathrm{D}}$ is inactivated) but unchanged in the distal part (because $I_{\mathrm{D}}$ is not inactivated by the somatic depolarization). As a result, synaptic efficacy is enhanced for the proximal synapse (red inward current) but not for the distal synapse (blue inward current).

ral precision of EPSP-spike coupling by reducing the time window in which an action potential can be elicited (200, 418).

\section{Backward axonal integration}

Voltage changes in the somatic compartment modify release properties at the nerve terminal, and the effect is reciprocal. Small physiological voltage changes at the nerve terminal affect action potential initiation (400). In their recent study, Paradiso and $\mathrm{Wu}(400)$ have shown that small subthreshold depolarizations $(<20 \mathrm{mV})$ of the calyx of Held produced by current injection or by the afterdepolarization (ADP) of a preceding action potential were found to decrease the threshold for action potential generated by local stimulation $400-800 \mu \mathrm{m}$ from the nerve terminal. Conversely, a small hyperpolarization of the nerve terminal $(<15 \mathrm{mV})$ produced either by current injection or the AHP increased the threshold for spike initiation. Thus this elegant study showed for the first time that axonal membrane, like dendrites, can backpropagate signals generated in the nerve terminal. Presynaptic $\mathrm{GABA}_{\mathrm{A}}$ currents originating in the axon have been recently identified in the cell body of cerebellar interneurons (535). Thus axonal GABAergic activity can probably influence somatic excitability in these neurons, further supporting the fact that axonal and somatodendritic compartments are not electrically isolated.

The functional importance of axonal integration is clear, but many questions remain open. The three examples where hybrid (analog-digital) signaling in the axon has been observed are glutamatergic neurons [CA3 pyramidal neurons (460), granule cells (5), and L5 pyramidal neurons $(291,489)]$. Do axons of GABAergic interneurons also support hybrid axonal signaling? A study indicates that this is not the case at synapses established by parvalbumin-positive fast-spiking cells that display delayed firing and pyramidal neurons in cortical layer 2-3 (117, 217). However, the equilibrium between excitation and inhibition probably needs to be preserved in cortical circuits, and one cannot exclude that hybrid axonal signaling may exist in other subclasses of cortical or hippocampal GABAergic interneurons. In cerebellar interneurons, GABA release is facilitated by subthreshold depolarization of the presynaptic soma (110). Can inhibitory postsynaptic potentials spread down the axon, and if so, how do they influence synaptic release? In dendrites, voltagegated channels amplify or attenuate subthreshold EPSPs. Do axonal voltage-gated channels also influence propagation of subthreshold potentials? Now that the axons of mammalian neurons are finally becoming accessible to 
direct electrophysiological recording, we can expect answers to all these questions.

\section{PROPAgAtion FAILURES}

One of the more unusual operations achieved by axons is selective conduction failure. When the action potential fails to propagate along the axon, no signal can reach the output of the cell. Conduction failure represents a powerful process that filters communication with postsynaptic neurons (549). Propagation failures have been observed experimentally in various axons including vertebrate spinal axons $(38,301)$, spiny lobster or crayfish motoneurons (230, 231, 241, 401, 496), leech mechanosensory neurons $(28,29,234,541,572)$, thalamocortical axons (151), rabbit nodose ganglion neurons (167), rat dorsal root ganglion neurons $(335,336)$, neurohypophysial axons $(55,172)$, and hippocampal pyramidal cell axons (144, 364, 498). However, some axons in the auditory pathways are capable of sustaining remarkably high firing rates, with perfect entrainment occurring at frequencies of up to $1 \mathrm{kHz}$ (467). Several factors determine whether propagation along axons fails or succeeds.

\section{A. Geometrical Factors: Branch Points and Swellings}

Although the possibility that propagation may fail at branch points was already discussed by Krnjevic and Miledi (301), the first clear indication that propagation is perturbed by axonal branch points came from the early studies on spiny lobster, crayfish, and leech axons (230, 231, 401, 496, 497, 541, 572). The large size of invertebrate axons allowed multielectrode recordings upstream and downstream of the branch point. For example, in lobster axons, conduction across the branch point was found to fail at frequencies above $30 \mathrm{~Hz}$ (Fig. 11A, Ref. 230). The block of conduction occurred specifically at the branch point because the parent axon and one of the daughter branches continued to conduct action potentials. Failures appeared first in the thicker daughter branch, but they could be also observed in the thin branch at higher stimulus frequency. In the leech, conduction block occurs at central branch points where fine axons from the periphery meet thicker axons (572). Branch point failures have been observed or suspected to occur in a number of mammalian neurons (144, 151, 167).

Propagation failures also occur when the action potential enters a zone with an abrupt change in diameter. This occurs with en passant boutons $(72,272,581)$ but also when impulses propagating along the axon enter the soma $(12,185,336)$. For instance, in the metacerebral cell of the snail, propagation failures have been observed when a spike enters the cell body (Fig. 11B; Ref. 12).

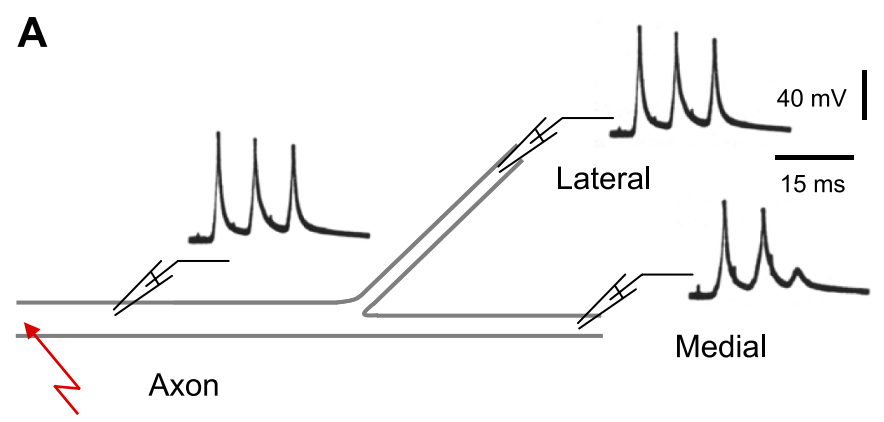

B

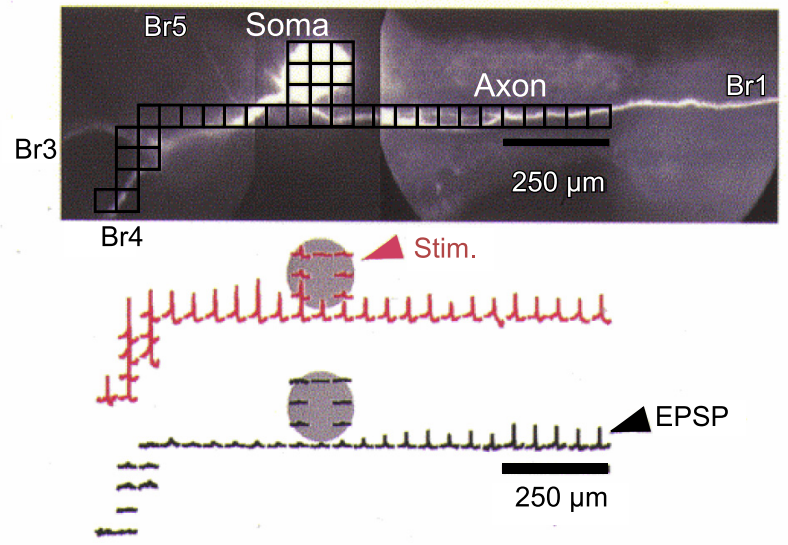

FIG. 11. Propagation failures in invertebrate neurons. A: propagation failure at a branch point in a lobster axon. The main axon and the medial and lateral branches are recorded simultaneously. The repetitive stimulation of the axon (red arrow) at a frequency of $133 \mathrm{~Hz}$ produces a burst of full spike amplitude in the axon and in the lateral branch but not in the medial branch. Note the electrotonic spikelet in response to the third stimulation. [Adapted from Grossman et al. (230), with permission from Wiley-Blackwell.] $B$ : propagation failure at the junction between an axonal branch and the soma of a snail neuron (metacerebral cell). The neuron was labeled with the voltage-sensitive styryl dye JPW1114. The propagation in the axonal arborization was analyzed by the local fluorescence transients due to the action potential. The recording region is indicated by an outline of a subset of individual detectors, superimposed over the fluorescence image of the neuron in situ. When the action potential was evoked by direct stimulation of the soma, it propagated actively in all axonal branches (red traces). In contrast, when the action potential was evoked by the synaptic stimulation (EPSP) of the right axonal branch (Br1), the amplitude of the fluorescent transient declined when approaching the cell body, indicating a propagation failure (black traces). [Adapted from Antic et al. (12), with permission from John Wiley \& Sons.]

These failures result because the electrical load is significantly higher on the arriving action potential, and the current generated by the parent axon is not sufficient to support propagation (reviewed in Ref. 470). Simulations show that at geometrical irregularities the propagating action potential is usually distorted in amplitude and width, and local conduction velocity can be changed. For instance, an abrupt increase in axon diameter causes a decrease in both velocity and peak amplitude of the action potential, whereas a step decrease in diameter has the opposite local effects on these two parameters (221, $226,272,337,338,348,349,403)$. In fact, the interplay between the total longitudinal current produced by the 
action potential and the input impedance of the axon segments ahead of the action potential determines the fate of the propagating action potential. The case of the branch point has been studied in detail $(219,221,583)$. The so-called 3/2 power law developed by Rall describes an ideal relationship between the geometry of mother and daughter branches $(221,424,426)$. A geometrical parameter (the geometrical ratio, GR) has been defined as follows: $\mathrm{GR}=\left(d^{3 / 2}\right.$ daughter $1+d^{3 / 2}$ daughter 2$) / d^{3 / 2}$ mother , where $d_{\text {daughter } 1}$ and $d_{\text {daughter } 2}$ are the diameters of the daughter branches and $d_{\text {mother }}$ is the diameter of the parent axon.

For GR $=1$, impedances match perfectly and spikes propagate in both branches. If GR $>1$, the combined electrical load of the daughter branches exceeds the load of the main branch. In other words, the active membrane of the mother branch may not be able to provide enough current to activate both branches. If GR $>10$, conduction block occurs in all daughter branches (404). For $1<$ GR $<10$, the most common situation by far, propagation past the branch point occurs with some delay. All these conclusions are only true if the characteristics of the membrane are identical, and any change in ion channel density may positively or negatively change the safety factor at a given branch point. The amplification of the propagating action potential by sodium channels in the mossy fiber bouton is able to counteract the geometrical effects and speeds up the propagation along the axon (179). Details on the experimental evaluation of GR at axon branch points has been reviewed elsewhere (139).

\section{B. Frequency-Dependent Propagation Failures}

Depending on the axon type, conduction failures are encountered following moderate $(10-50 \mathrm{~Hz})$ or high-frequency $(200-300 \mathrm{~Hz}$ ) stimulation of the axon. For instance, a frequency of $20-30 \mathrm{~Hz}$ is sufficient to produce conduction failures at the neuromuscular terminal arborization (301) or at the branch point of spiny lobster motoneurons (230). These failures are often seen as partial spikes or spikelets that are electrotonic residues of full action potentials. The functional consequences of conduction failures might be important in vivo. For example, in the leech, propagation failures produce an effect similar to that of sensory adaptation. They represent a nonsynaptic mechanism that temporarily disconnects the neuron from one defined set of postsynaptic neurons and specifically routes sensory information in the ganglion $(234,339,541,572)$.

What are the mechanisms of frequency-dependent conduction failure? As mentioned above, the presence of a low safety conduction point such as a branch point, a bottleneck (i.e., an axon entering the soma) or an axonal swelling determines the success or failure of conduction. However, these geometrical constraints are not sufficient to fully account for all conduction failures, and additional factors should be considered. The mechanisms of propagation failure can be grouped in two main categories.

First, propagation may fail during repetitive axon stimulation as a result of a slight depolarization of the membrane. At spiny lobster axons, propagation failures were associated with a $10-15 \%$ reduction of the action potential amplitude in the main axon and a membrane depolarization of 1-3 $\mathrm{mV}$ (230). These observations are consistent with potassium efflux into the peri-axonal space induced by repetitive activation. In most cases, the membrane depolarization produced by external accumulation of potassium ions around the axon probably contributes to sodium channel inactivation. In fact, hyperpolarization of the axon membrane or local application of physiological saline with a low concentration of potassium in the vicinity of a block can restore propagation in crayfish axons (496). Elevation of the extracellular potassium concentration produced conduction block in spiny lobster axons (231). However, this manipulation did not reproduce the differential block induced by repetitive stimulation, as failures occurred simultaneously in both branches (230). Interestingly, conduction could also be restored by the elevation of intracellular calcium concentration. Failures were also induced with a lower threshold when the electrogenic $\mathrm{Na}^{+} / \mathrm{K}^{+}$pump was blocked with ouabain. Thus differential conduction block could be explained as follows. During high-frequency activation, potassium initially accumulates at the same rate around the parent axon and the daughter branches. Sodium and calcium accumulate more rapidly in the thin branch than in the thick branch because of the higher surface-to-volume ratio. Thus the $\mathrm{Na}^{+} / \mathrm{K}^{+}$pump is activated and extracellular potassium is lowered, more effectively around the thin branch (231). Accumulation of extracellular potassium has also been observed in the olfactory nerve (178) and in hippocampal axons (416), and could similarly be at the origin of unreliable conduction.

Propagation failures have also been reported in the axon of Purkinje neurons under high regimes of stimulation (283, 372; Fig. 12A). In this case, the cell body was recorded in whole cell configuration, whereas the signal in the axon was detected in cell-attached mode at a distance up to $800 \mu \mathrm{m}$ from the cell body. Propagation was found to be highly reliable for single spikes at frequencies below $200 \mathrm{~Hz}$ (failures were observed above 250 $\mathrm{Hz}$ ). In physiological conditions, Purkinje cells typically fire simple spikes well below $200 \mathrm{~Hz}$, and these failures are unlikely to be physiologically relevant (196). However, Purkinje cells also fire complex spikes (bursts) following stimulation of the climbing fiber. The instantaneous frequency during these bursts may reach $800 \mathrm{~Hz}$ (283, 372). Interestingly, complex spikes did not propagate reliably in Purkinje cell axons. Generally, only the first and the last spike of the burst propagate. The failure 

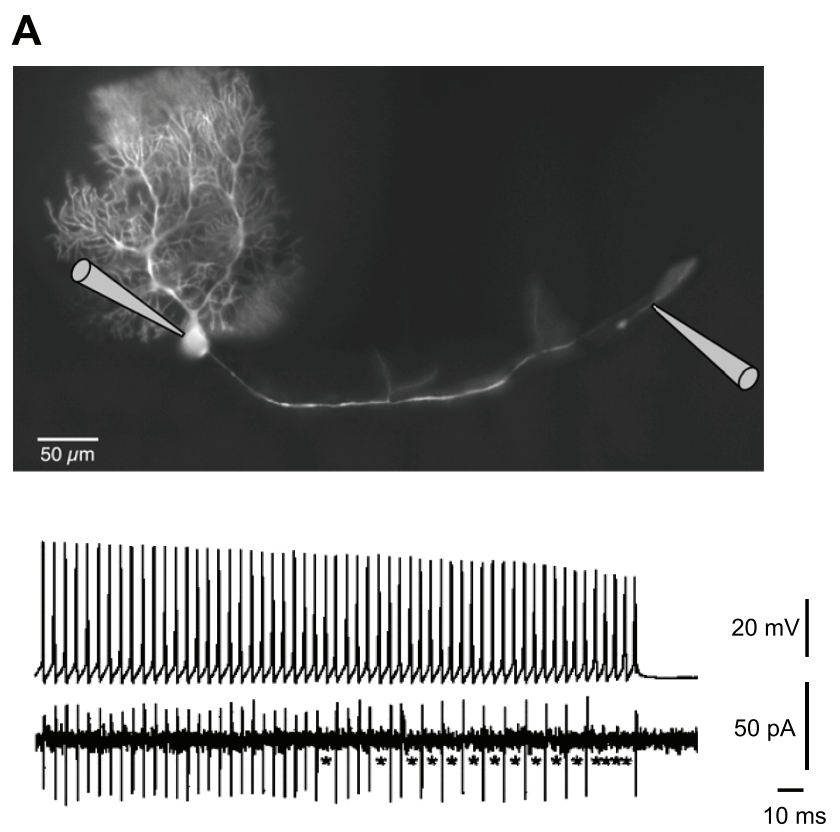

B
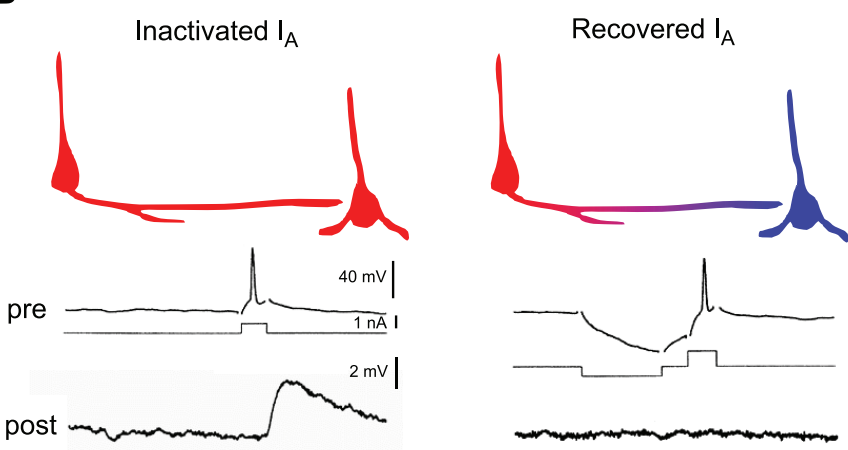

FIG. 12. Propagation failures in mammalian axons. A: propagation failures in a Purkinje cell axon. Top: fluorescent image of a Purkinje cell filled with the fluorescent dye Alexa 488. The locations of the somatic and axonal recordings are indicated schematically. [Adapted from Monsivais et al. (372).] $B$ : gating of action potential propagation by the potassium current $I_{\mathrm{A}}$. Left: at resting membrane potential, presynaptic $I_{\mathrm{A}}$ was inactivated and the action potential evoked in the presynaptic cell propagated and elicited an EPSP in the postsynaptic cell. Right: following a brief hyperpolarizing prepulse, presynaptic $I_{\mathrm{A}}$ recovered from inactivation and blocked propagation. Consequently, no EPSP was evoked by the presynaptic action potential. [Adapted from Debanne et al. (144), with permission from Nature Publishing Group.]

rate of the complex spike is very sensitive to membrane potential, and systematic failures occur when the cell body is depolarized (372). The limit of conduction has not been yet fully explored in glutamatergic cell axons, but conduction failures have been reported when a CA3 pyramidal neuron fire at $30-40 \mathrm{~Hz}$ during a long plateau potential (362). Thus the conduction capacity seems much more robust in inhibitory cells compared with glutamatergic neurons. However, this study was based on extracellular recordings, and the apparent conduction failures may result from detection problems. In fact, very few failures were observed with whole cell recordings in neocortical pyramidal neurons (489). Furthermore, the robustness of spike propagation along axons of inhibitory neurons will require further studies.

Propagation failures induced by repetitive stimulation may also result from hyperpolarization of the axon. Hyperpolarization-induced conduction block has been observed in leech $(339,541,572)$, locust (247), and mammalian axons $(55,167)$. In this case, axonal hyperpolarization opposes spike generation. Activity-dependent hyperpolarization of the axon usually results from the activation of the $\mathrm{Na}^{+}-\mathrm{K}^{+}$-ATPase and/or the activation of calcium-dependent potassium channels. Unmyelinated axons in the PNS, for example, vagal C-fibers, hyperpolarize in response to repeated action potentials $(445,446)$ as a result of the intracellular accumulation of $\mathrm{Na}^{+}$and the subsequent activation of the electrogenic $\mathrm{Na}^{+} / \mathrm{K}^{+}$pump (42, $445,446)$. In crayfish axons, this hyperpolarization may amount to $5-10 \mathrm{mV}$ (42). The blockade of the $\mathrm{Na}^{+}-\mathrm{K}^{+}$ATPase with ouabain results in axon depolarization, probably as a consequence of posttetanic changes in extracellular potassium concentration. In the leech, hyperpolarization-dependent conduction block occurs at central branch points in all three types of mechanosensory neurons in the ganglion: touch $(\mathrm{T})$, pressure $(\mathrm{P})$, and nociceptive $(\mathrm{N})$ neurons. In these neurons, hyperpolarization is induced by the $\mathrm{Na}^{+}-\mathrm{K}^{+}$-ATPase and by cumulative activation of a calcium-activated potassium conductance. It is interesting to note that the conduction state can be changed by neuromodulatory processes. 5-HT decreases the probability of conduction block in $\mathrm{P}$ and $\mathrm{T}$ cells, probably by a reduction of the hyperpolarization (350).

Hyperpolarization-dependent failures have also been reported in axons of hypothalamic neurons (from paraventricular and supraoptic nuclei) that run into the neurohypophysis. The morphology of their boutons is unusual in that their diameter varies between 5 and $15 \mu \mathrm{m}$ (581). In single axons, propagation failures are observed at stimulation rates greater than $12 \mathrm{~Hz}$ and are concomitant with a hyperpolarization of $4 \mathrm{mV}$ (55). Here, the induced hyperpolarization of the neuron results from activation of the calcium-dependent BK potassium channels.

Several recent studies indicate that the hyperpolarization produced by repetitive stimulation could be dampened by hyperpolarization-induced cationic current $\left(I_{\mathrm{h}}\right)$ $(42,498)$. This inward current is activated at resting membrane potential and produces a tonic depolarization of the axonal membrane (42). Thus reduction of this current induces a hyperpolarization and perturbs propagation. The pharmacological blockade of $I_{\mathrm{h}}$ by ZD-7288 or by external cesium can in fact produce more failures in Schaffer collateral axons (498). The peculiar biophysical properties of $I_{\mathrm{h}}$ indicate that it may limit large hyperpolarizations or depolarizations produced by external and internal accumulation of ions. In fact, hyperpolarization 
of the axon will activate $I_{\mathrm{h}}$, which in turn produces an inward current that compensates the hyperpolarization (42). Reciprocally, this compensatory mechanism is also valid for depolarization by removing basal activation of $I_{\mathrm{h}}$. In addition, activity-induced hyperpolarization of the axonal membrane may modulate the biophysical state of other channels that control propagation.

\section{Frequency-Independent Propagation Failures}

Action potential propagation in some axon collaterals of cultured CA3 pyramidal neurons can be gated by activation of presynaptic A-type $\mathrm{K}^{+}$current, independently of the frequency of stimulation $(144,295)$. Synaptic transmission between monosynaptically coupled pairs of CA3-CA3 or CA3-CA1 pyramidal cells in hippocampal slice cultures can be blocked if a brief hyperpolarizing current pulse is applied a few milliseconds before the induction of the action potential in the presynaptic neuron (Fig. 12B). This regulation is observed in synaptic connections that have no transmission failures, therefore indicating that the lack of postsynaptic response is the consequence of a conduction failure along the presynaptic axon. In contrast to axonal integration where transmitter can be gradually regulated by the presynaptic membrane potential, transmission is all or none. Interestingly, failures can also be induced when the presynaptic hyperpolarizing current pulse is replaced by a somatic IPSP $(144,295)$. When presynaptic cells are recorded with a microelectrode containing 4-aminopyridine (4-AP), a blocker of $I_{\mathrm{A}}$-like conductances, failures are abolished, indicating that $I_{\mathrm{A}}$ gates action potential propagation (see also Ref. 389). Because A-channels are partly inactivated at the resting membrane potential, their contribution during an action potential elicited from the resting membrane potential is minimal, and the action potential propagates successfully from the cell body to the nerve terminal. In contrast, A-channels recover from inactivation with a transient hyperpolarization and impede successful propagation to the terminal.

Propagation failures have been induced in only $30 \%$ of cases (144), showing that propagation is generally reliable in hippocampal axons $(341,342,422)$. In particular, $I_{\mathrm{A}}$-dependent conduction failures have been found to occur at some axon collaterals but not at others (144). With the use of a theoretical approach, it has been shown that failures occur at branch points when A-type $\mathrm{K}^{+}$channels are distributed in clusters near the bifurcation (295). Perhaps because these conditions are not fulfilled in layer II/III neocortical neurons $(128,289)$ and in dissociated hippocampal neurons (341), this form of gating has not been reported in these cell types. It would be interesting to explore the actual distribution of $\mathrm{K}^{+}$channel clusters near branch points using immunofluorescence methods.
Functionally, this form of gating may determine part of the short-term synaptic facilitation that is observed during repetitive presynaptic stimulation. Apparent paired-pulse facilitation could be observed because the first action potential fails to propagate but not the second spike, as a result of inactivation of A-type $\mathrm{K}^{+}$current (145). A recent study suggests that repetitive burst-induced inactivation of A-type $\mathrm{K}^{+}$channels in the axons of cortical cells projecting onto accumbens nucleus leads to short-term synaptic potentiation through an increased reliability of spike propagation (98).

\section{REFLECTION OF ACTION POTENTIAL PROPAGATION}

Branch points are usually considered as frequency filters, allowing separate branches of an axon to activate their synapses at different frequencies. But another way that a neuron's branching pattern can affect impulse propagation is by reflecting the impulse $(221,402,428)$. Reflection (or reverse propagation) occurs when an action potential is near failure (221). This form of axonal computation has been well described in leech mechanosensory neurons (Fig. 13A; Refs. 28, 29) in which an unexpected event occurs when conduction is nearly blocked: the action potential that has nearly failed to invade the thick branch of the principal axon sets up a local potential that propagates backwards. Reflection occurs because impulses are sufficiently delayed as they travel through the branch point. Thus, when the delay exceeds the refractory period of the afferent axon, the impulse will propagate backwards as well as forwards, creating a reflection. This phenomenon can be identified electrophysiologically at the cell body of the P neuron because action potentials that reflect had a longer initial rising phase (or "foot"), indicating a delay in traveling through the branch point. This fast double firing in the thin branch of mechanosensory neurons has important functional consequences. It facilitates synaptic transmission at synapses formed by this axon and postsynaptic neurons by a mechanism of paired-pulse facilitation with the orthodromic spike and the antidromic action potential that reflected at the branch point (Fig. 13A). Reflection is not limited to $\mathrm{P}$ cells but also concerns $\mathrm{T}$ cells (28). Interestingly, the facilitation of synaptic transmission also affects the chemical synapse between the $\mathrm{P}$ cell and the $\mathrm{S}$ neuron, a neuron that plays an essential role in sensitization, a nonassociative form of learning (29).

Reflected propagation is not restricted to mechanosensory neurons of the leech but has also been noted in the axon of an identified snail neuron (12). Reflection has not yet been definitively reported in mammalian axons (270), but it has been demonstrated in dendrites. In mitral cells of the mammalian olfactory bulb, both conduction 
A

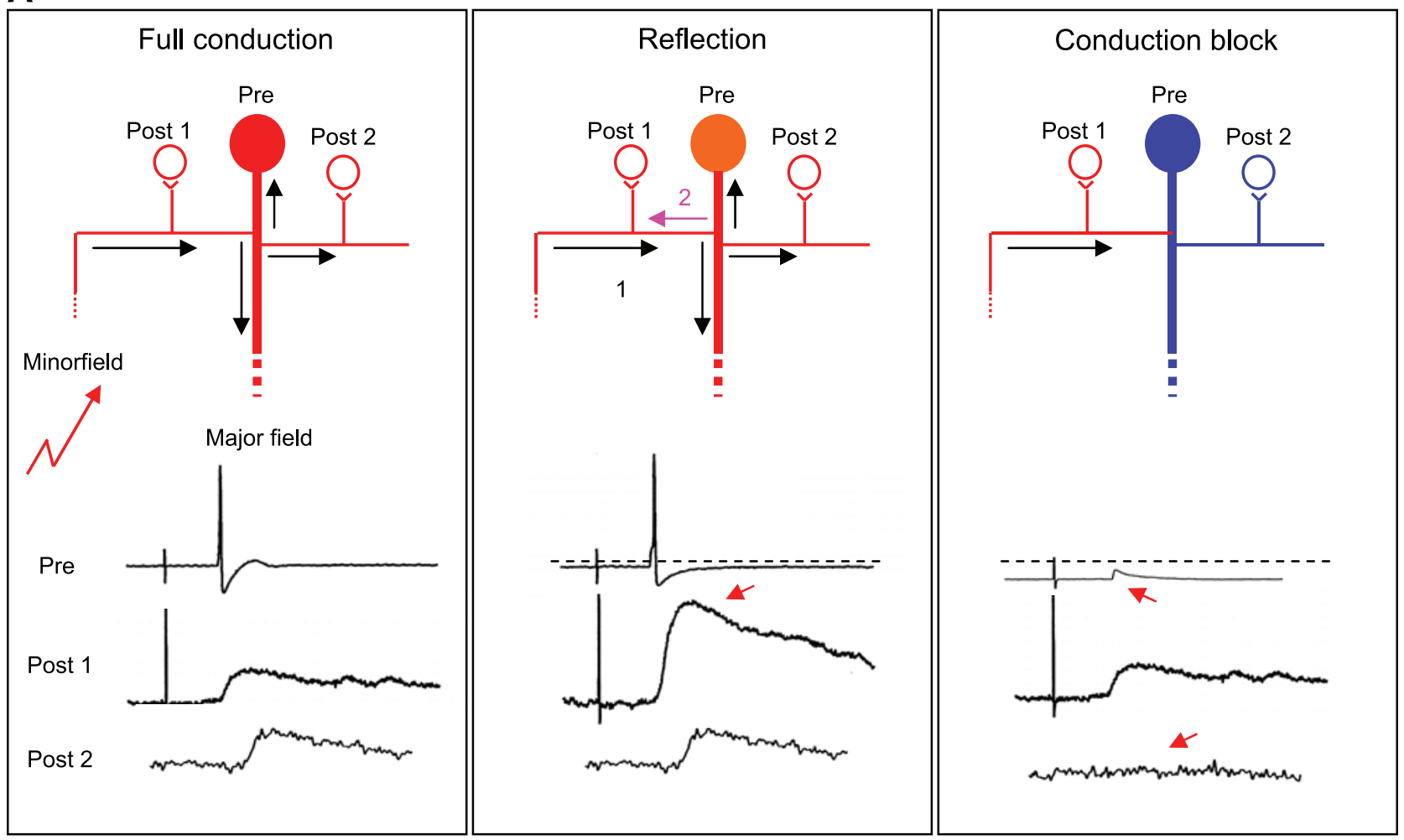

B
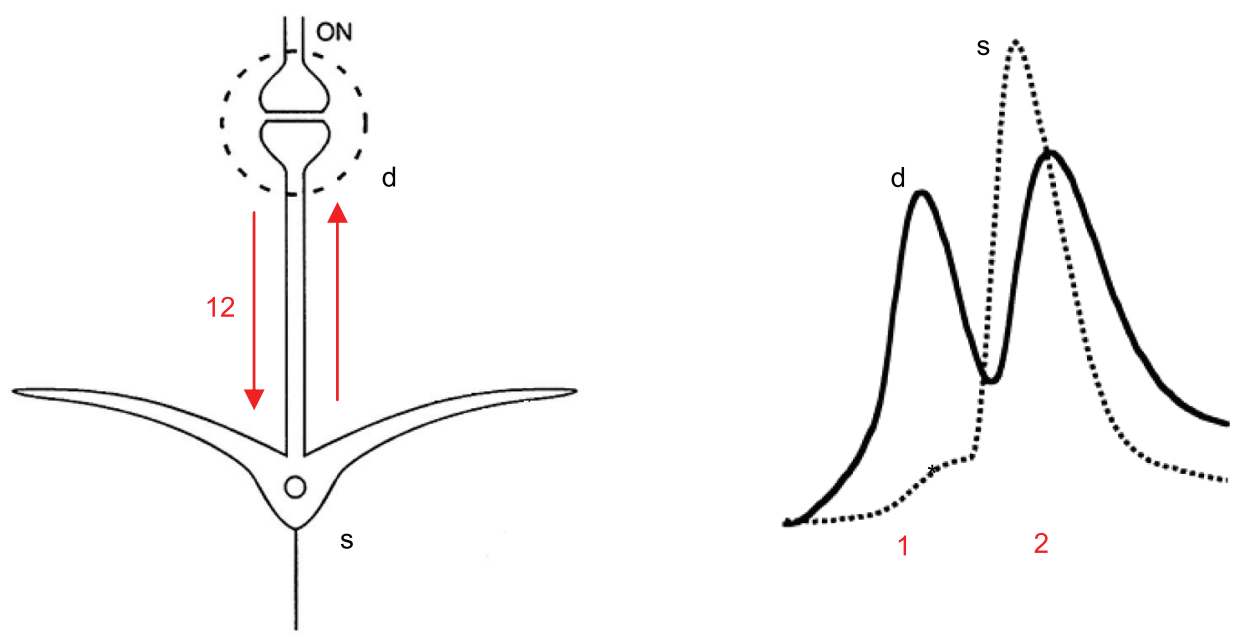

FIG. 13. Reflection of action potentials. A: reflection and conduction block produce multilevel synaptic transmission in mechanosensory neurons of the leech. Left column: an action potential initiated by anterior minor field stimulation invades the whole axonal arborization (red) and evokes an EPSP in all postsynaptic cells. Middle column: following repetitive stimulation, the cell body is slightly hyperpolarized (orange) and the same stimulation induces a reflected action potential at the branch point between the left branch and the principal axon. The reflected action potential (pink arrow 2) stimulates the presynaptic terminal on postsynaptic cell 1 twice, thus enhancing synaptic transmission (arrow). Right column: when the cell body is further hyperpolarized (blue), the stimulation of the minor field now produces an action potential that fails to propagate at the branch point. The failed spike is seen as a spikelet at the cell body (upward arrow). No postsynaptic response is evoked in postsynaptic cell 2 (downward arrow). [Adapted from Baccus et al. $(28,29)$.] $B$ : reflection of action potential propagation in the presynaptic dendrite of the mitral cell. The dendritic and somatic compartments are recorded simultaneously. An action potential (1) initiated in the dendrite (d) fails to propagate towards the soma ( $\mathrm{s}$, dotted trace), is then regenerated at the soma (2), and propagates back to the dendrite, thus producing a double dendritic spike (thick trace in the inset). The asterisk marks the failing dendro-somatic spike. [Adapted from Chen et al. (108).] 
failures (107) and reflection (108) have been observed for impulses that are initiated in dendrites (Fig. 13B). Propagation in dendrites of mitral cells is rather unusual compared with classical dendrites. Like axons, it is highly active, and no decrement in the amplitude of the AP is observed between the soma and the dendrite (60). In addition, mitral cell dendrites are both pre- and postsynaptic elements. "Ping-pong" propagation has been observed following near failure of dendritic action potentials evoked in distal primary dendrites (108). Forward dendritic propagation of an action potential can be evoked by an EPSP elicited by a strong stimulation of the glomerulus. This particular form of propagation may fail near the cell body when the soma is slightly hyperpolarized. For an intermediate range of membrane potential, the action potential invades the soma and may trigger a back-propagating AP, which is seen as a dendritic double spike in the primary dendrite. The function of reflected propagation is not yet definitively established, but when axonal output is shut down by somatic inhibition, the primary dendrite of the mitral cell may function as a local interneuron affecting its immediate environment. Reflection of fast action potentials has also been observed in dendrites of retinal ganglion cells (544).

\section{SPIKE TIMING IN THE AXON}

\section{A. Delay Imposed by Axonal Length}

Axonal conduction introduces a delay in the propagation of neuronal output, and axonal arborization might transform a temporal pattern of activity in the main axon into spatial patterns in the terminals (113). Axonal delay initially depends on the velocity of the action potential in axons (generally between $0.1 \mathrm{~m} / \mathrm{s}$ in unmyelinated axons and $100 \mathrm{~m} / \mathrm{s}$ in large myelinated axons) which directly results from the diameter of the axon and the presence of a myelin sheath. Axonal delays may have crucial functional consequences in the integration of sensory information. In the first relay of the auditory system of the barn owl, differences in the axonal conduction delay from each ear, which in this case depends on the differences in axonal length, produce sharp temporal tuning of the binaural information that is essential for acute sound localization (Fig. 14A; Refs. 95, 96, 358).

What is the functional role of axonal delay in network behavior? Theoretical work shows that synchronization of cortical columns and network resonance both depend
A

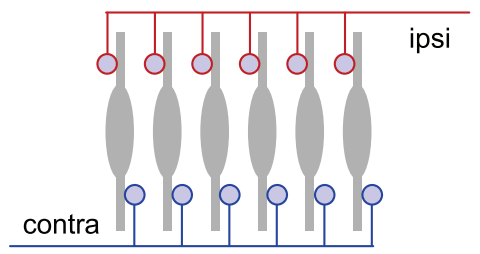

B
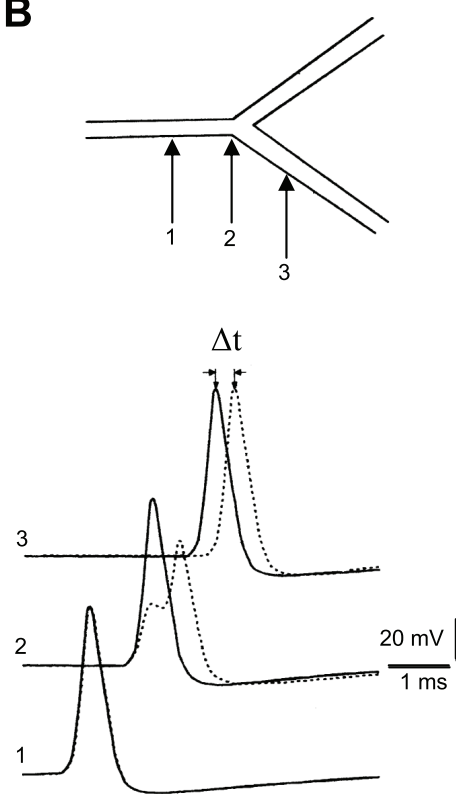

C
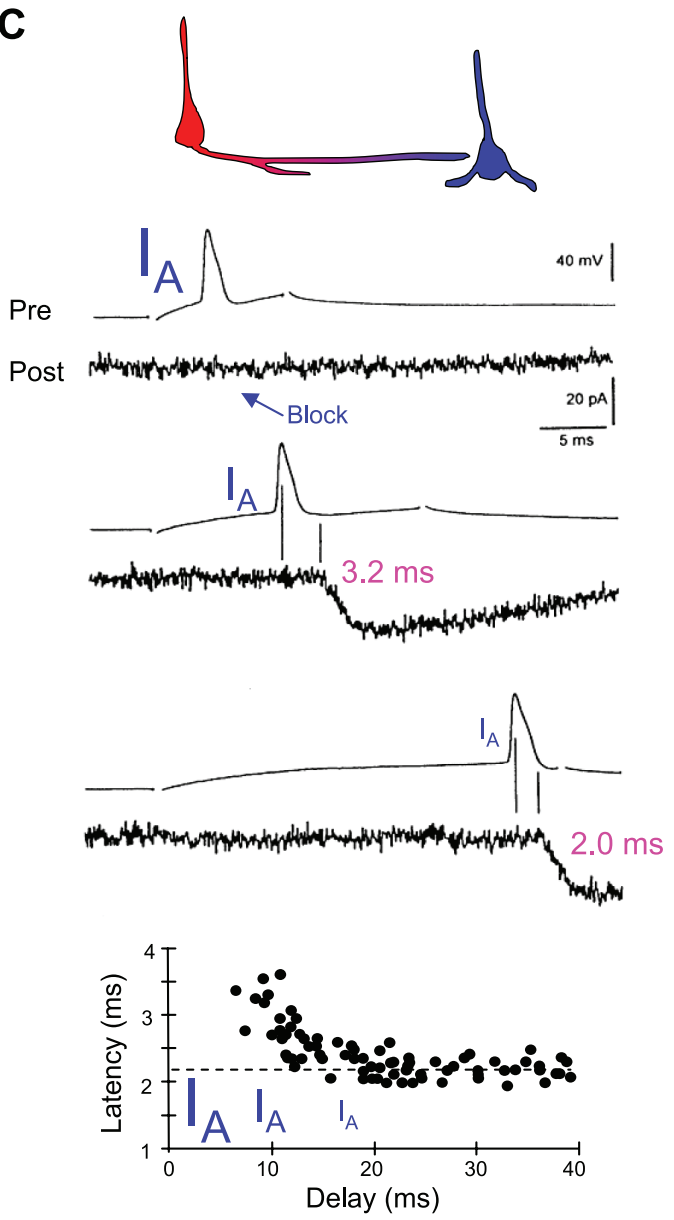

FIG. 14. Axonal propagation and spike timing. $A$ : delay lines in the auditory system of the barn owl. Each neuron from the nucleus laminaris receives an input from each ear. Note the difference in axonal length from each side. [Adapted from Carr and Konishi (96).] B: comparison of the delay of propagation introduced by a branch point with GR $>1$ (dashed traces) versus a branch point with perfect impedance matching (GR $=1$, continuous traces). Top: schematic drawing of a branched axon with 3 points of recording. At the branch point with $\mathrm{GR}=8$, the shape of the action potential is distorted and the propagation displays a short latency $(\Delta \mathrm{t})$. [Adapted from Manor et al. (349).] $C$ : propagation failures in hippocampal cell axons are associated with conduction delays. The presynaptic neuron was slightly hyperpolarized with constant current to remove inactivation of the A-current $\left(I_{\mathrm{A}}\right)$. A presynaptic action potential induced with a short delay after onset of the depolarizing pulse did not elicit an EPSC in the postsynaptic cell because of the large activation of $I_{\mathrm{A}}$. Increasing the delay permitted action potential propagation because $I_{\mathrm{A}}$ was reduced during the action potential. For complete inactivation of $I_{\mathrm{A}}$ (bottom pair of traces), latency decreased. [Adapted from Debanne et al. (144), with permission from Nature Publishing Group.] 
on axonal delay $(87,344)$. A recent theoretical study emphasizes the importance of axonal delay in the emergence of poly-synchronization in neural networks (271). In most computational studies of storage capacity, axonal delay is totally ignored, but in fact, the interplay between axonal delays and synaptic plasticity based on timing (spike-timing-dependent plasticity, STDP) generates the emergence of polychronous groups (i.e., strongly interconnected groups of neurons that fire with millisecond precision). Most importantly, the number of groups of neurons that fire synchronously exceeds the number of neurons in a network, resulting in a system with massive memory capacity (271).

However, differences in axonal delay may be erased to ensure synchronous activity. A particularly illustrative example is given by the climbing fiber inputs to cerebellar Purkinje cells. Despite significant differences in the length of individual olivocerebellar axons, the conduction time is nearly constant because long axons are generally thicker. Thus this compensatory mechanism allows synchronous activation of Purkinje cells with millisecond precision (518). Similarly, the eccentricity of X-class retinal ganglion cells within the retina is compensated by their conduction velocity to produce a nearly constant conduction time (507). Thus, regardless of the geometrical constraints imposed by retinal topography, a precise spatiotemporal representation of the retinal image can be maintained in the visual relay.

\section{B. Delays Imposed by Axonal Irregularities and Ion Channels}

In addition to this axonal delay, local changes in the geometry of the axon produce an extra delay. The presence of axonal irregularities such as varicosities and branch points reduces conduction velocity (Fig. 14B). This reduction in conduction velocity occurs as a result of a high geometrical ratio (GR; see sect. VIA). The degree of temporal dispersion has been simulated in the case of an axon from the somatosensory cortex of the cat (349). The delay introduced by high GR branch points could account for a delay of $0.5-1 \mathrm{~ms}$ (349). But this extra delay appears rather small compared with the delay imposed by the conduction in axon branches with variable lengths (in the range of 2-4 $\mathrm{ms})$.

A third category of delay in conduction can be introduced during repetitive stimulation or during the activation of specific ion channels. Thus the magnitude of this delay is usually variable. It has been measured in a few cases. In lobster axons, the conduction velocity of the axon was lowered by $\sim 30 \%$ following repetitive stimulation (231). In dorsal root ganglion neurons, the latency of conducted spikes was found to be enhanced by $\sim 1 \mathrm{~ms}$ following antidromic paired-pulse stimulation of the axon (336). Com- putational studies indicate that this delay may also result from a local distortion of the action potential shape. Activitydependent delays may have significant consequences on synaptic transmission. For instance, the synaptic delay was found to increase by 1-2 ms during repetitive stimulation of crayfish motor neurons (241). Monosynaptic connections to motoneurons show an increase in synaptic latency concomitant with the synaptic depression induced by repetitive stimulation at $5-10 \mathrm{~Hz}$, which induced near-propagation failures (510). Similarly, a longer synaptic delay has been measured between connected hippocampal cells when conduction nearly fails, due to reactivation of A-type potassium channels (Fig. 14C; Ref. 144). Thus axonal conduction may introduce some noise into the temporal pattern of action potentials produced at the initial segment. At the scale of a nerve, delays in individual axons introduce a temporal dispersion of conduction, suggesting a stuttering model of propagation (374).

Synaptic timing at L5-L5 or CA3-CA3 unitary connections is largely determined by presynaptic release probability (70). Synaptic latency is inversely correlated with the amplitude of the postsynaptic current, and changes in synaptic delay in the range of 1-2 ms are observed during paired-pulse and long-term plasticity involving regulation of presynaptic release (70). Probability of transmitter release is not the only determinant of synaptic timing, however. The waveform of the axonal spike also plays a critical role. The enlargement of the axonal spike by a Kv channel blocker significantly prolongs synaptic latency at L5-L5 synapses (71). The underlying mechanism results from the shift in the presynaptic calcium current. Because the presynaptic action potential overshoots at approximately $+50 \mathrm{mV}$, the calcium current develops essentially during the repolarizing phase of the presynaptic spike $(23,59,279,328,454)$. Thus the spike broadening produced by 4-AP delays the calcium current and subsequently shifts glutamate release towards longer latencies. Physiologically, spike broadening in the axon may occur when $\mathrm{Kv}$ channels are inactivated during repetitive axonal stimulation and may participate in the stabilization of synaptic delay (71).

The probabilistic nature of voltage-gated ion channels (i.e., channel noise) may also affect conduction time along fibers below $0.5 \mu \mathrm{m}$ diameter. A simulation study indicates that four distinct effects may corrupt propagating spike trains in thin axons: spikes being added, deleted, jittered, or split into subgroups (186). The local variation in the number of $\mathrm{Na}^{+}$channels may cause microsaltatory conduction.

\section{Ephaptic Interactions and Axonal Spike Synchronization}

Interactions between neighboring axons were first studied by Katz and Schmitt $(280,281)$ in crab. The passage of an impulse in one axonal fiber produced a sub- 
threshold change in excitability in the adjacent fiber. As the action potential approaches in the active axon, the excitability of the resting fiber was first reduced, and then quickly enhanced $(280,288)$. This effect results from the depolarization of the resting axon by the active axon because it generates locally an extracellular potential of a few millivolts. Interactions of this type are called ephaptic (from the Greek for "touching onto," Ref. 20). They are primarily observed when the extracellular conductance is reduced $(37,280)$. This condition is fulfilled, for instance, in bundles of unmyelinated axons where the periaxonal space is minimal, as in olfactory nerves $(62,68)$. Ephaptic interactions between axons have also been observed in frog sciatic nerve (288) and in demyelinated spinal axons of dystrophic mice (436).

One of the most interesting features of ephaptic interaction between adjacent axons is that the conduction velocity in neighboring fibers might be unified, thus synchronizing activity in a bundle of axons. If one action potential precedes the other by a few milliseconds, it accelerates the conduction rate of the lagging action potential in the other axon (37, 280; Fig. 15). This phenomenon occurs because the ephaptic potential created in the adjacent fiber is asymmetrical. When the delay between the two spikes is small ( $\sim 1-2 \mathrm{~ms}$; Ref. 37$)$, the depolarizing phase of the ephaptic potential facilitates spike generation and increases conduction velocity. However, perfectly synchronized action potentials decrease the conduction velocity in both branches because of the initial hyperpolarizing phase of the ephaptic potentials. Synchronization can only occur if the individual velocities differ only slightly and are significant for a sufficient axonal length (280). Does such synchronization also occur in mammalian axons? There is no evidence for this yet, but modeling studies indicate that the relative location of nodes of Ranvier on two adjacent myelinated axons might also determine the degree of temporal synchrony between fibers $(57,440)$. On small unmyelinated axons, ephaptic interaction between axons is predicted to be very small (254), but future research in this direction might reveal a powerful means to thoroughly synchronize neuronal activity downstream of the site of action potential initiation.

\section{Electric Coupling in Axons and Fast Synchronization}

Fast communication between neurons is not only ensured by chemical synapses, but electrical coupling has been reported in a large number of cell types including inhibitory cortical interneurons (249). In the hippocampus, one type of high-frequency oscillation $(100-200 \mathrm{~Hz})$ called "ripple" arises from the high-frequency firing of inhibitory interneurons and phase-locked firing of many
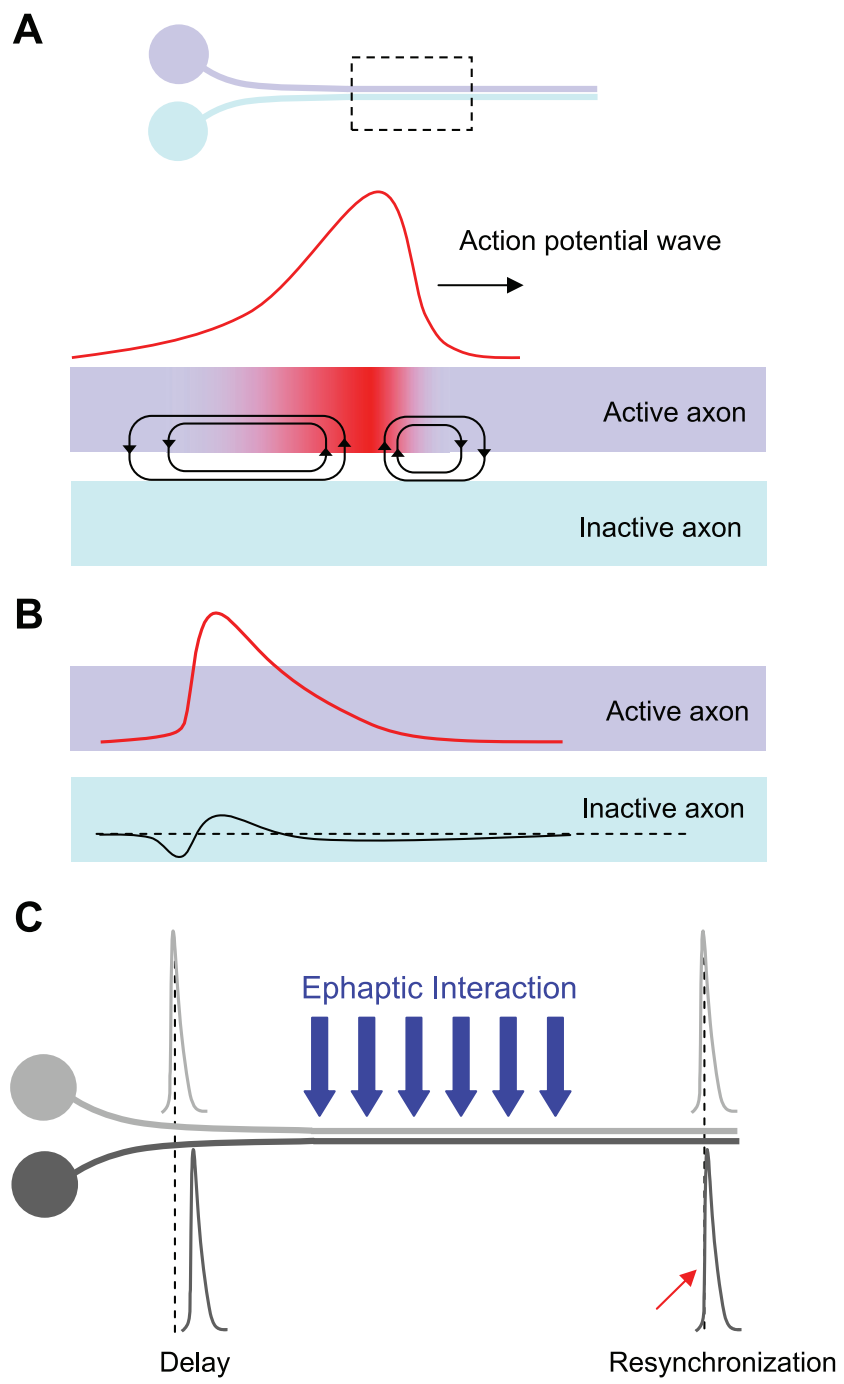

FIG. 15. Ephaptic interaction in axons. A: local circuit diagram in a pair of adjacent axons. The red area indicates the "active region." The action currents produced by the action potential penetrates the inactive axon. $B$ : schematic representation of resynchronization of action potentials in a pair of adjacent axons. While the spikes propagate along the axons, the initial delay between them becomes reduced. [Adapted from Barr and Plonsey (37) and Katz and Schmitt (280).]

CA1 neurons (533). Some of the properties of ripple oscillation are, however, difficult to explain. First, the oscillations are so fast (near $200 \mathrm{~Hz}$ ) that synchrony across many cells would be difficult to achieve through chemical synaptic transmission. In addition, ripples persist during pharmacological blockade of chemical transmission in vitro (162). While some inhibitory interneurons may synchronize a large number of pyramidal cells during the ripple (286), a significant part of the synchronous activity could be mediated by axo-axonal electrical synaptic contacts through gap junctions (464). Antidromic stimulation of a neighboring axon elicits a small action potential, a spikelet with a fast rate of rise (near $180 \mathrm{mV} / \mathrm{ms}$ ). Spikelets can be evoked at the rate of a ripple $(200 \mathrm{~Hz})$, and they are blocked by TTX or by the gap junction blocker 
carbenoxolone. Simultaneous recording from the axon and cell body showed that the spikelet first traversed the axon prior to invading the soma and the dendrites. Finally, the labeling of pyramidal neurons with rhodamine, a small fluorescent molecule, showed dye coupling in adjacent neurons that was initiated through the axon (464). Thus the function of the axon is not limited to the conduction of the impulses to the terminal, and information may process between adjacent pyramidal neurons through electrical synapses located close to their axon hillock.

A similar mechanism of electrical coupling between proximal axons of Purkinje cells is supposed to account for very fast oscillations $(>75 \mathrm{~Hz})$ in the cerebellum. Very fast cerebellar oscillations recorded in cerebellar slices are indeed sensitive to gap junction blockers (368). In addition, spikelets and fast prepotentials eliciting full spikes are observed during these episodes. In fact, the simulation of a cerebellar network where Purkinje cells are sparsely linked though axonal gap junctions replicates the experimental observations (534).

Cell-cell communication through axons of CA1 pyramidal neurons has recently been suggested in vivo (181). Using the newly developed technique of in vivo whole cell recording in freely moving rats $(321,322,352)$, the group of Michael Brecht found that most records from CA1 cells $(\sim 60 \%)$ display all-or-none events, with electrophysiological characteristics similar to spikelets resulting from electrical coupling in the axon. These events have a fast rise time $(<1 \mathrm{~ms})$ and a biphasic decay time. They occur during ripples as bursts of three to six events (181).

\section{ACTIVITY-DEPENDENT PLASTICITY OF AXON MORPHOLOGY AND FUNCTION}

\section{A. Morphological Plasticity}

The recent development of long-term time lapse imaging in vitro and in vivo (255) has revealed that axon morphology is highly dynamic. Whereas the large-scale organization of the axonal arborization remains fairly stable over time in adult central neurons, a subset of axonal branchlets can undergo impressive structural rearrangements in the range of a few tens of micrometers (review in Ref. 256). These rearrangements affect both the number and size of en passant boutons as well as the complexity of axonal arborization. For instance, the hippocampal mossy fiber terminals are subject to dramatic changes in their size and complexity during in vitro development and in the adult in vivo following exposure to enriched environment (203, 204, 216). The turnover of presynaptic boutons in well identified Schaffer collateral axons is increased following induction of LTD in vitro (44). Finally, in an in vitro model of traumatic epilepsy, transection between the CA3 and CA1 region induces axonal sprouting associated with an increase in the density of boutons per unit length (360).

Axonal reorganization has also been reported in vivo. In the visual cortex, a subset of geniculo-cortical axonal branches can undergo structural rearrangements during development (14) and in the adult (508) or following activity deprivation $(239,240,554)$. Similar observations have been reported in the barrel cortex during development (417) and in the adult mice (137). However, one should note that the magnitude of axonal rearrangements is much larger during the critical period of development. In the adult mice cerebellum, transverse, but not ascending branches of climbing fibers are dynamic, showing rapid elongation and retraction (383). The motility of axonal branches is clearly demonstrated in all these studies, and it certainly reflects dynamic rewiring and functional changes in cortical circuits. Neuronal activity seems to play a critical role in the motility of the axon, but the precise mechanisms are not clearly understood. For instance, stimulation of the axon freezes dynamic changes in cerebellar climbing fibers in vivo (383). Similarly, the fast motility of axonal growth cone of hippocampal neurons in vitro is reduced by stimulation of GluR6 kainate receptors or electrical stimulation and depends on axonal calcium concentration (266). In contrast, the slow remodeling of local terminal arborization complexes of the mossy fiber axon is reduced when $\mathrm{Na}^{+}$channel activity is blocked with TTX (204).

Electrical activity not only determines axon morphology but also controls induction of myelination in developing central and peripheral axons. For instance, blockade of $\mathrm{Na}^{+}$channel activity with TTX reduces the number of myelinated segment and the number of myelinating oligodendrocytes, whereas increasing neuronal excitability has the opposite effects (149). In contrast, electrical stimulation of dorsal root ganglion neurons delays myelin formation (509). In this case, ATP released by active axons is subsequently hydrolyzed to adenosine that stimulates adenosine receptors in Schwann cells and freezes their differentiation. Neuronal activity is also thought to determine the maintenance of the myelin sheath in adult axons. In the hindlimb unloading model, myelin thickness is tightly controlled by motor activity (93). Myelin is thinner in axons controlling inactive muscles but thicker in hyperactive axons.

\section{B. Functional Plasticity}

Beyond morphological rearrangements, the axon is also able to express many forms of functional plasticity $(520,557)$. In fact, several lines of evidence suggest that ion channel activity is highly regulated by synaptic or neuronal activity (reviews in Refs. 135, 493, 582). There- 
fore, some of the axonal operations described in this review could be modulated by network activity. Axonal plasticity can be categorized into Hebbian and homeostatic forms of functional plasticity according to the effects of the induced changes in neuronal circuits. Hebbian plasticity usually serves to store relevant information and to some extent destabilizes neuron ensembles, whereas homeostatic plasticity is compensatory and stabilizes network activity within physiological bounds $(420,539)$.

\section{Hebbian plasticity of axonal function}

There are now experimental facts suggesting that Hebbian functional plasticity exists in the axon. For instance, the repetitive stimulation of Schaffer collateral axons at $2 \mathrm{~Hz}$ leads to a long-lasting lowering of the antidromic activation threshold (361). Although the precise expression mechanisms have not been characterized here, this study suggests that axonal excitability is persistently enhanced if the axon is strongly stimulated. Furthermore, LTP and LTD are respectively associated with increased and decreased changes in intrinsic excitability of the presynaptic neuron $(205,324)$. These changes imply retrograde messengers that target the presynaptic neuron. Although these changes are detected in the cell body, the possibility that ion channels located in the axon are also regulated cannot be excluded. Two parallel studies have recently reported a novel form of activity-dependent plasticity in a subclass of inhibitory interneurons of the cortex and hippocampus containing neuropeptide Y $(476,523)$. Stimulation of the interneuron at $20-40 \mathrm{~Hz}$ leads to an increase in action potential firing lasting several minutes. In both studies, the persistent firing is consistent with the development of an ectopic spike initiation zone in the distal region of the axon.

\section{Homeostatic axonal plasticity}

The expression of axonal channels might be regulated by chronic manipulation of neuronal activity according to the homeostatic scheme of functional plasticity. For instance, blocking neuronal activity by TTX enhances both the amplitude of the transient $\mathrm{Na}^{+}$current (150) and the expression of $\mathrm{Na}^{+}$channels in hippocampal neurons (16). Although the subcellular distribution of $\mathrm{Na}^{+}$channels was not precisely determined in these studies, they might be upregulated in the axon. Indeed, axon regions that become silent because of acute demyelination express a higher density of $\mathrm{Na}^{+}$channels which eventually allows recovery of active spike propagation $(69,195,555)$. Activity deprivation not only enhances intrinsic excitation but also reduces the intrinsic neuronal brake provided by voltage-gated $\mathrm{K}^{+}$channels $(131,141,150)$. Chronic inactivation of neuronal activity with TTX or synaptic blockers inhibits the expression of Kv1.1, Kv1.2, and Kv1.4 potassium channels in the cell body and axon of cultured hippocampal neurons (229). Although the functional consequences were not analyzed here, this study suggests that downregulation of Kv1 channels would enhance neuronal excitability and enlarge axonal spike width.

The position of the AIS relative to the cell body is also subject to profound activity-dependent reorganization (Fig. 16A). In a recent study, Grubb and Burrone (232, 233) showed that brief network-wide manipulation of electrical activity determines the position of the AIS in hippocampal cultured neurons. The AIS identified by its specific proteins ankyrin-G and $\beta$-IV-spectrin is moved up to $17 \mu \mathrm{m}$ distally (i.e., without any change in the AIS length) when activity is increased by high external potas-
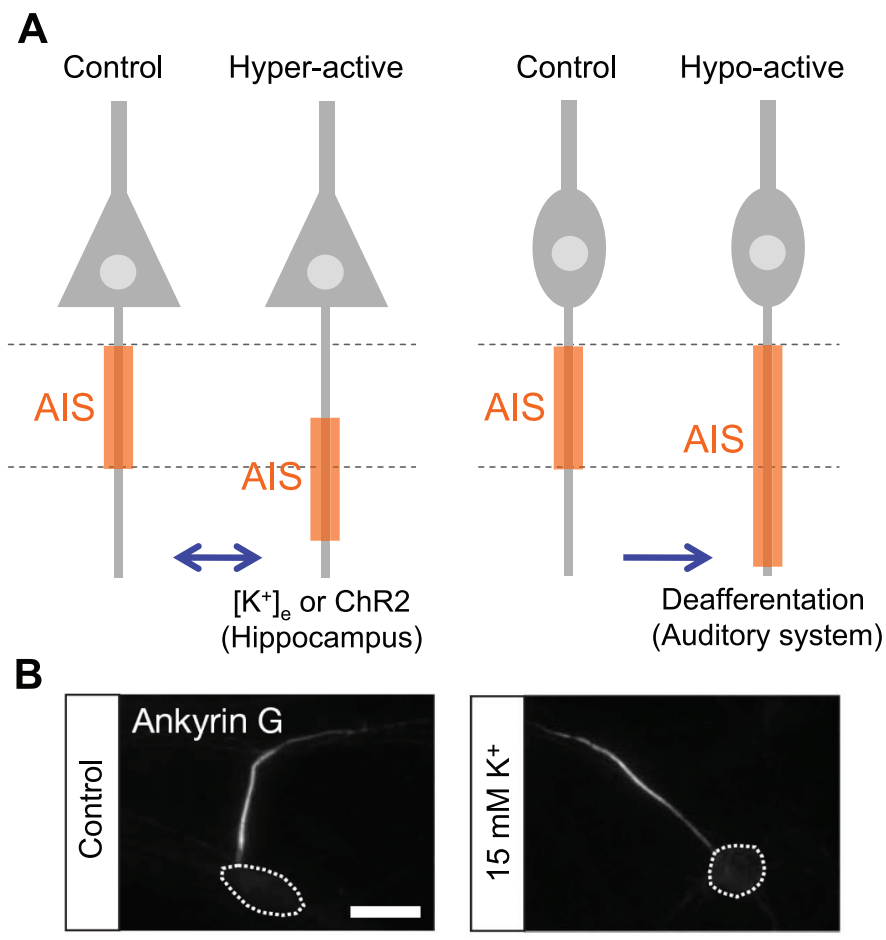

C
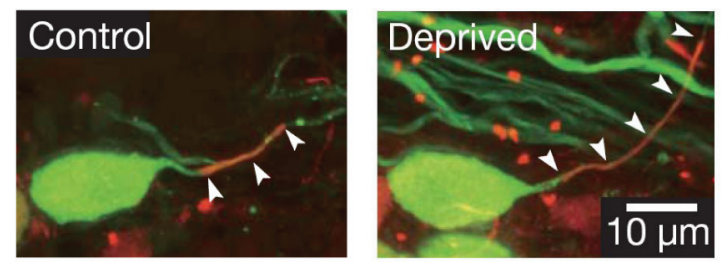

FIG. 16. Activity-dependent plasticity of AIS. A: scheme of the homeostatic regulation of AIS location in cultured hippocampal neurons (left) and in brain stem auditory neurons (right). AIS is moved distally following chronic elevation of activity by high external $\mathrm{K}^{+}$or photostimulation of neurons expressing the light-activated cation channel channelrhodopsin 2 (ChR2) (left). AIS length is augmented in chick auditory neurons following cochlea removal (right). $B$ : ankyrin G label in control neurons and in neurons treated with $15 \mathrm{mM} \mathrm{K}^{+}$during $48 \mathrm{~h}$ (scale bar: $20 \mu \mathrm{m}$ ). [From Grubb and Burrone (232), with permission from Nature Publishing Group.] $C$ : AIS plasticity in chick auditory neurons. Sodium channels have been immunolabeled with pan-Na channel antibody. Neurons from deprived auditory pathway display longer AIS (right) than control neurons (left). [From Kuba et al. (304), with permission from Nature Publishing Group.] 
sium or illumination of neurons transfected with channelrhodopsin-2 during $48 \mathrm{~h}$ (232; Fig. 16B). The relocation of the AIS is reversible and depends on T- and L-type calcium channels, suggesting that intra-axonal calcium may control the dynamic of the AIS protein scaffold. This bidirectional plasticity might be a powerful means to adjust the excitability of neurons according to the homeostatic rule of plasticity (539). In fact, neurons with proximal AIS are generally more excitable than those with distal AIS, suggesting that shifting the location of the AIS distally elevates the current threshold for action potential generation $(232,298)$. Thus these data indicate that AIS location is a mechanism for homeostatic regulation of neuronal excitability.

Homeostatic AIS plasticity might be a general rule and may account for the characteristic frequency-dependent distribution of sodium channels along the axon of chick auditory neurons $(302,303)$. In neurons that preferentially analyze high auditory frequencies $(\sim 2 \mathrm{kHz})$, sodium channels are clustered at $20-50 \mu \mathrm{m}$ from the soma, whereas they are located in the proximal part of the axon in neurons that detect lower auditory frequencies ( $\sim 600 \mathrm{~Hz}$; Ref. 302). A recent study from Kuba and coworkers (304) directly demonstrates the importance of afferent activity in AIS position in chick auditory neurons. Removing cochlea in young chicks produces an elongation of the AIS in nucleus magnocellularis neurons without affecting its distance from the cell body (Fig. 16C; Ref. 304). This regulation is associated with a compatible increase in the whole cell $\mathrm{Na}^{+}$currents.

Axonal excitability is also homeostatically tuned on short-term scales. Sodium channel activity is downregulated by many neuromodulators and neurotransmitters including glutamate that classically enhances neuronal activity $(92,94)$. Although further studies will be required to precisely determine the location of the regulated $\mathrm{Na}^{+}$ channels, it is nevertheless tempting to speculate that AIS excitability might be finely tuned.

\section{PATHOLOGIES OF AXONAL FUNCTION}

Beyond Wallerian degeneration that may be caused by axon sectioning, deficits in axonal transport $(121,138)$, or demyelination (381), the axon is directly involved in at least two large families of neurological disorders. Neurological channelopathies such as epilepsies, ataxia, pain, myotonia, and periodic paralysis usually result from dysfunction in ion channel properties or targeting $(130,306$, $409,461)$. The major consequences of these alterations are dysfunctions of neuronal excitability and/or axonal conduction (297). In addition, some forms of CharcotMarie-Tooth disease affect primarily the axon (297, 367, $519)$. They mainly lead to deficits in axonal propagation (297, 519).

\section{A. Axonal Diseases Involving Ion Channels}

\section{Epilepsies}

Many ion channels expressed in the axons of cortical neurons are mutated in human epilepsies, and dysfunction of the AIS is often at the origin of epileptic phenotypes (562). For instance, mutations of the gene SCN1A encoding Nav1.1 cause several epileptic phenotypes including generalized epilepsy with febrile seizure plus (GEFS+) and severe myoclonic epilepsy of infancy (SMEI) $(39,114,182$; Fig. 17). Some of these mutations do not produce a gain of function (i.e., hyperexcitability) as expected in the case of epilepsy, but rather a loss of function (505). Since Nav1.1 channels are highly expressed in the axons of GABAergic neurons (394), a decrease in excitability in inhibitory neurons will enhance excitability of principal neurons that become less inhibited. Mice lacking $S C N 1 A$ display spontaneous seizures because the sodium current is reduced in inhibitory interneurons but not in pyramidal cells (576). Similarly, deletions or mutations in Kv1.1 channels produce epi-

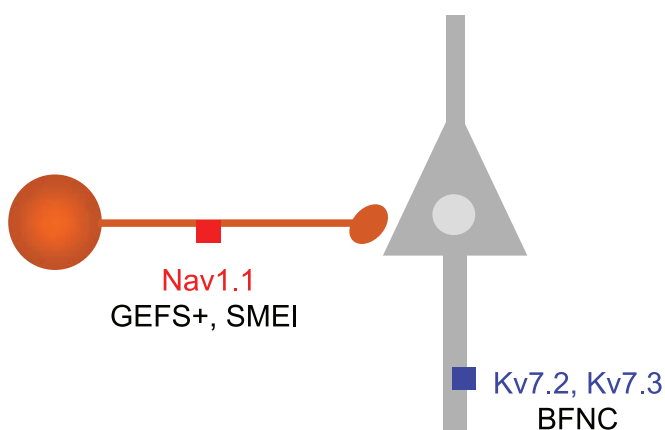
BFNC

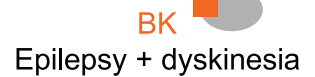

FIG. 17. Axonal channelopathies in cortical circuits. The possible roles of axonal ion channels implicated in epilepsy are illustrated schematically. Mutations in Nav1.1 from axons of GABAergic interneurons produce a loss of Na-channel function (i.e., reduced excitability of inhibitory interneurons but increased network activity) that might underlie epilepsy with febrile seizure plus (GEFS+) or severe myoclonic epilepsy of infancy (SMEI). Mutations in Kv7.2/7.3 channels lead to a loss of function (i.e., an increase in excitability of principal neurons) and may result in benign familial neonatal convulsions (BFNC). Deletions or mutations in Kv1.1 increase neuronal excitability and produce episodic ataxia type 1 . 
lepsy (495) and episodic ataxia type 1 (EA1), characterized by cerebellar incoordination and spontaneous motorunit activity (80). Mutations in KCNQ2/3 (Kv7.2/Kv7.3) channels produce several forms of epilepsy such as benign familial neonatal convulsions (BNFC; Refs. 406, 466, 492; Fig. 17). Some mutations may also target ion channels located in axon terminals. For instance, a missense mutation in the KCNMA1 gene encoding BK channels is associated with epilepsy and paroxysmal dyskinesia (164; Fig. 15).

Epilepsies may also be acquired following an initial seizure. For instance, many epileptic patients display graduated increases in the frequency and strength of their crises, indicating that epilepsy might be acquired or memorized by neuronal tissue. The cellular substrate for this enhanced excitability is thought to be long-lasting potentiation of excitatory synaptic transmission $(32,146)$, but enhanced neuronal excitability might be also critical (43, $50,64,517)$. These changes in excitability are generally proepileptic, but additional work will be required to determine whether axonal channels specifically contribute to acquired epilepsy phenotypes.

In addition to epilepsy, mutations in the SCNA1A or CACNA1A gene can also lead to cases of familial hemiplegic migraine; these mutations have mixed effects when studied in expression systems that could explain how they concur to cortical spreading depression $(103,408)$.

\section{Axonal channelopathies in the PNS}

Mutations in axonal channels may be involved in several diseases that affect the PNS. For instance, pain disorders are often associated with mutations of the SCN9A gene encoding the alpha subunit of Nav1.7, that cause either allodynia (i.e., burning pain; Refs. 189, 571) or analgesia (129). Pain is usually associated with a gain of function of Nav1.7 (i.e., lower activation threshold or reduced inactivation; Refs. 189, 238).

\section{B. Axonal Diseases Involving Myelin}

\section{Multiple sclerosis}

Multiple sclerosis (MS) is characterized by multiple attacks on CNS myelin that may lead to sensory (principally visual) and/or motor deficits $(532,555)$. MS is generally diagnosed in the young adult (before 40), and the progression of the disease often alternates phases of progression and remission where the patient recovers because compensatory processes occur, such as $\mathrm{Na}^{+}$channel proliferation in the demyelinated region (555). Althought the etiology of MS is multiple with hereditary, infectious, and environmental factors, the most important determinant of MS is dysregulation of the immune system including autoimmune diseases directed against myelin proteins. The main consequence is a partial or total loss of myelin that prevents axonal conduction in axons of the optic nerves or corticospinal tracts.

\section{Charcot-Marie-Tooth disease}

Charcot-Marie-Tooth (CMT) disease affects myelin of PNS axons and constitutes a highly heterogeneous group of genetic diseases. These diseases generally invalidate molecular interactions between axonal and glial proteins that stabilize myelin produced by Schwann cells. The most frequent forms, CMT1A, CMT1B, and CMT1X, are caused by mutations in genes which encode three components of the myelin sheath, peripheral myelin protein-22 (PMP22), myelin protein zero (MPZ), and connexin 32 , respectively (519).

\section{Hereditary neuropathy with liability to pressure palsies}

Hereditary neuropathy with liability to pressure palsies (HNPP) is a genetic disease that results from a deficiency in the gene coding for PMP22 (104). HNPP is characterized by focal episodes of weakness and sensory loss and is associated with abnormal myelin formation leading to conduction blocks (31).

\section{CONCLUDING REMARKS}

\section{A. Increased Computational Capabilities}

Axons achieve several fundamental operations that go far beyond classical propagation. Like active dendrites, axons amplify and integrate subthreshold and suprathreshold electrical signals $(5,144,179,291,489)$. In addition, the output message can be routed in selective axonal pathways at a defined regime of activity. The consequences of this are not yet well understood in mammalian axons, but branch point failures may participate in the elaboration of sensory processing in invertebrate neurons (234). Axonal propagation may also bounce back at a branch point or at the cell body, but at present, there are only a handful of examples showing reflected propagation $(12,28,29,108)$. Reflected impulses may limit the spread of the neuronal message and enhance synaptic transmission. Theoretical and experimental studies indicate that reflection of action potentials could occur in axons that display large swellings or a branch point with high GR. Moreover, axonal delay is important to set network resonance (344) and increase storage capacity in neuronal networks (271). Finally, axonal coupling through ephaptic interactions or gap junctions may precisely synchronize network activity $(448,464)$. All these operations increase the computational capabilities of axons and affect the 
dynamics of synaptic coupling. Many pieces of the puzzle are, however, still missing.

The computational capabilities of axons might be further extended by another unexpected and important feature: their capacity to express both morphological and functional plasticity. There is now evidence for Hebbian and homeostatic long-term axonal plasticities that might further enhance the computational capacity of the circuits $(232,233,304)$. Thus activity-dependent plasticity is not restricted to the input side of the neuron (i.e., its dendrites and postsynaptic differentiation), but it may also directly involve axonal function.

\section{B. Future Directions and Missing Pieces}

In the recent past, most (if not all) of our knowledge about axonal computation capabilities was derived from experiments on invertebrate neurons or from computer simulations (470). The use of paired-recording techniques $(140,144)$ and the recent spread of direct patch-clamp recordings from the presynaptic terminal $(5,58,179,432)$ or from the axon $(259,291,292,488-490)$ suggest that the thin mammalian axon will yield up all its secrets in the near future. There are good reasons to believe that, combined with the development of high-resolution imaging techniques like multiphoton confocal microscopy (128, 193, 194, 289), second-harmonic generation microscopy (160) and voltage-sensitive dyes (12, 74, 196, 215, 228, 327, $396,397,580$ ), this technique will be a powerful tool to dissect the function of axons. Development of nanoelectronic recording devices will also probably offer promising solutions to solve the problem of intracellular recording from small-diameter axons (530).

Axonal morphology and the subcellular localization of ion channels play crucial roles in conduction properties and propagation failures or reflected propagation may result from the presence of axonal irregularities such as varicosities and branch points. However, detailed quantitative analysis of the morphometry of single axons combined with the quantitative immunostaining of sodium channels as used recently by Lorincz and Nusser (333) will be needed. The use of recently developed molecular tools to target defined channel subunits towards specific axonal compartments could be of great help in determining their role in axonal propagation.

Fine temporal tuning can be achieved by axons. Differences in axonal length in the terminal axonal tuft introduce delays of several milliseconds. Is temporal scaling of action potential propagation in the axonal arborization relevant to the coding of neuronal information? Differential conduction delays in axonal branches participate in precise temporal coding in the barn owl auditory system $(95,96,358)$. But the role of axonal delays has only been studied in artificial neural networks $(87,271,344)$ or in vitro neuronal circuits (33), and additional work will have to be done to describe its implication in hybrid (i.e., neuron-computer) or in in vivo networks. Furthermore, understanding the conflict faced by cortical axons between space (requirement to connect many different postsynaptic neurons) and time (conduction delay that must be minimized) will require further studies (83).

Local axonal interactions like ephaptic coupling and gap-junction coupling allow very fast synchronization of activity in neighboring neurons. Surprisingly, little experimental effort has been devoted to ephaptic interactions between axons. This mechanism represents a powerful means to precisely synchronize output messages of neighboring neurons. Perhaps ephaptic interactions between parallel axons could compensate the "stuttering conduction" that is introduced by axonal varicosities and branch points (374). The implications of these mechanisms in synchronized activity will have to be determined in axons that display favorable geometrical arrangement for ephaptic coupling (i.e., fasciculation over a sufficient axonal length). Callosal axons, mossy fibers, and Schaffer collaterals are possible candidates.

In conclusion, we report here evidence that beyond classical propagation many complex operations are achieved by the axon. The axon displays a high level of functional flexibility that was not expected initially. Thus it may allow a fine tuning of synaptic strength and timing in neuronal microcircuits. There are good reasons to believe that after the decade of the dendrites in the 1990s, a new era of axon physiology is now beginning.

\section{ACKNOWLEDGMENTS}

We thank M. Seagar for constant support, P. Giraud for providing confocal images of labeled neurons, and S. Binczak for helpful discussion. We thank J. J. Garrido, A. Marty, M. Seagar, and F. Tell for helpful comments on the manuscript and the members of D. Debanne's lab for positive feedback.

Address for reprint requests and other correpondence: D. Debanne, Université de la Méditerranée, Faculté de médecine secteurnord, IFR 11, Marseille, F-13916 France(e-mail: dominique. debanne@univmed.fr).

\section{GRANTS}

This work was supported by Institut National de la Santé et de la Recherche Médicale, Centre National de la Recherche Scientifique, Ministry of Research (doctoral grants to E. Campanac and A. Bialowas), Fondation pour la Recherche Médicale (to E. Campanac), and Agence Nationale de la Recherche (to D. Debanne and G. Alcaraz).

\section{DISCLOSURES}

No conflicts of interest, financial or otherwise, are declared by the authors. 
1. Abraham WC, Gustafsson B, Wigstrom H. Long-term potentiation involves enhanced synaptic excitation relative to synaptic inhibition in guinea-pig hippocampus. J Physiol 394: 367-380, 1987.

2. Ahern GP, Hsu SF, Klyachko VA, Jackson MB. Induction of persistent sodium current by exogenous and endogenous nitric oxide. J Biol Chem 275: 28810-28815, 2000.

3. Aizenman CD, Linden DJ. Rapid, synaptically driven increases in the intrinsic excitability of cerebellar deep nuclear neurons. Nat Neurosci 3: 109-111, 2000.

4. Alle H, Geiger JR. Analog signalling in mammalian cortical axons. Curr Opin Neurobiol 18: 314-320, 2008.

5. Alle H, Geiger JR. Combined analog and action potential coding in hippocampal mossy fibers. Science 311: 1290-1293, 2006.

6. Alle H, Geiger JR. GABAergic spill-over transmission onto hippocampal mossy fiber boutons. J Neurosci 27: 942-950, 2007.

7. Alle H, Roth A, Geiger JR. Energy-efficient action potentials in hippocampal mossy fibers. Science 325: 1405-1408, 2009.

8. Andersen P, Soleng AF, Raastad M. The hippocampal lamella hypothesis revisited. Brain Res 886: 165-171, 2000.

9. Andersen P, Sundberg SH, Sveen O, Swann JW, Wigstrom H. Possible mechanisms for long-lasting potentiation of synaptic transmission in hippocampal slices from guinea-pigs. J Physiol 302: 463-482, 1980

10. Anderson P, Storm J, Wheal HV. Thresholds of action potentials evoked by synapses on the dendrites of pyramidal cells in the rat hippocampus in vitro. J Physiol 383: 509-526, 1987.

11. Angstadt JD, Calabrese RL. A hyperpolarization-activated inward current in heart interneurons of the medicinal leech. $J \mathrm{Neu}$ rosci 9: 2846-2857, 1989.

12. Antic S, Wuskell JP, Loew L, Zecevic D. Functional profile of the giant metacerebral neuron of Helix aspersa: temporal and spatial dynamics of electrical activity in situ. J Physiol 527: 55-69, 2000.

13. Antonini A, Gillespie DC, Crair MC, Stryker MP. Morphology of single geniculocortical afferents and functional recovery of the visual cortex after reverse monocular deprivation in the kitten. $J$ Neurosci 18: 9896-9909, 1998

14. Antonini A, Stryker MP. Rapid remodeling of axonal arbors in the visual cortex. Science 260: 1819-1821, 1993.

15. Aponte Y, Lien CC, Reisinger E, Jonas P. Hyperpolarizationactivated cation channels in fast-spiking interneurons of rat hippocampus. J Physiol 574: 229-243, 2006.

16. Aptowicz CO, Kunkler PE, Kraig RP. Homeostatic plasticity in hippocampal slice cultures involves changes in voltage-gated $\mathrm{Na}^{+}$ channel expression. Brain Res 998: 155-163, 2004.

17. Arbuthnott ER, Boyd IA, Kalu KU. Ultrastructural dimensions of myelinated peripheral nerve fibers in the cat and their relation to conduction velocity. J Physiol 308: 125-157, 1980.

18. Armett CJ, Ritchie JM. The action of acetylcholine on conduction in mammalian non-myelinated fibres and its prevention by an anticholinesterase. J Physiol 152: 141-158, 1960.

19. Arnold DB. Actin and microtubule-based cytoskeletal cues direct polarized targeting of proteins in neurons. Sci Signal 2: pe49, 2009.

20. Arvanitaki A. Effects evoked in an axon by the activity of a contiguous one. J Neurophysiol 5: 89-108, 1942.

21. Astman N, Gutnick MJ, Fleidervish IA. Persistent sodium current in layer 5 neocortical neurons is primarily generated in the proximal axon. $J$ Neurosci 26: 3465-3473, 2006 .

22. Atherton JF, Wokosin DL, Ramanathan S, Bevan MD. Autonomous initiation and propagation of action potentials in neurons of the subthalamic nucleus. J Physiol 586: 5679-5700, 2008.

23. Augustine GJ, Charlton MP, Smith SJ. Calcium entry into voltage-clamped presynaptic terminals of squid. J Physiol 367: 143$162,1985$.

24. Awatramani GB, Price GD, Trussell LO. Modulation of transmitter release by presynaptic resting potential and background calcium levels. Neuron 48: 109-121, 2005.

25. Axmacher N, Miles R. Intrinsic cellular currents and the temporal precision of EPSP-action potential coupling in CA1 pyramidal cells. J Physiol 555: 713-725, 2004.
26. Azouz R, Gray CM. Cellular mechanisms contributing to response variability of cortical neurons in vivo. J Neurosci 19: 2209-2223, 1999.

27. Azouz R, Gray CM. Dynamic spike threshold reveals a mechanism for synaptic coincidence detection in cortical neurons in vivo. Proc Natl Acad Sci USA 97: 8110-8115, 2000

28. Baccus SA. Synaptic facilitation by reflected action potentials: enhancement of transmission when nerve impulses reverse direction at axon branch points. Proc Natl Acad Sci USA 95: 8345-8350, 1998.

29. Baccus SA, Burrell BD, Sahley CL, Muller KJ. Action potential reflection and failure at axon branch points cause stepwise changes in EPSPs in a neuron essential for learning. $J$ Neurophysiol 83: 1693-1700, 2000.

30. Baginskas A, Palani D, Chiu K, Raastad M. The H-current secures action potential transmission at high frequencies in rat cerebellar parallel fibers. Eur J Neurosci 29: 87-96, 2009.

31. Bai Y, Zhang X, Katona I, Saporta MA, Shy ME, O'Malley HA, Isom LL, Suter U, Li J. Conduction block in PMP22 deficiency. $J$ Neurosci 30: 600-608, 2010.

32. Bains JS, Longacher JM, Staley KJ. Reciprocal interactions between CA3 network activity and strength of recurrent collateral synapses. Nat Neurosci 2: 720-726, 1999.

33. Bakkum DJ, Chao ZC, Potter SM. Long-term activity-dependent plasticity of action potential propagation delay and amplitude in cortical networks. PLoS One 3: e2088, 2008.

34. Ballo AW, Bucher D. Complex intrinsic membrane properties and dopamine shape spiking activity in a motor axon. $J$ Neurosci 29 : 5062-5074, 2009.

35. Baranauskas G, Martina M. Sodium currents activate without a Hodgkin-and-Huxley-type delay in central mammalian neurons. $J$ Neurosci 26: 671-684, 2006.

36. Barnes AP, Polleux F. Establishment of axon-dendrite polarity in developing neurons. Annu Rev Neurosci 32: 347-381, 2009.

37. Barr RC, Plonsey R. Electrophysiological interaction through the interstitial space between adjacent unmyelinated parallel fibers. Biophys J 61: 1164-1175, 1992.

38. Barron DH, Matthews BH. Intermittent conduction in the spinal cord. J Physiol 85: 73-103, 1935.

39. Baulac S, Gourfinkel-An I, Picard F, Rosenberg-Bourgin M, Prud'homme JF, Baulac M, Brice A, LeGuern E. A second locus for familial generalized epilepsy with febrile seizures plus maps to chromosome 2q21-q33. Am J Hum Genet 65: 1078-1085, 1999.

40. Baumann N, Pham-Dinh D. Biology of oligodendrocyte and myelin in the mammalian central nervous system. Physiol Rev 81: 871-927, 2001.

41. Bean BP. The action potential in mammalian central neurons. Nat Rev Neurosci 8: 451-465, 2007.

42. Beaumont V, Zhong N, Froemke RC, Ball RW, Zucker RS. Temporal synaptic tagging by $\mathrm{I}(\mathrm{h})$ activation and actin: involvement in long-term facilitation and cAMP-induced synaptic enhancement. Neuron 33: 601-613, 2002.

43. Beck H, Yaari Y. Plasticity of intrinsic neuronal properties in CNS disorders. Nat Rev Neurosci 9: 357-369, 2008.

44. Becker N, Wierenga CJ, Fonseca R, Bonhoeffer T, Nagerl UV. LTD induction causes morphological changes of presynaptic boutons and reduces their contacts with spines. Neuron 60: 590-597, 2008.

45. Bender KJ, Ford CP, Trussell LO. Dopaminergic modulation of axon initial segment calcium channels regulates action potential initiation. Neuron 68: 500-511, 2010

46. Bender KJ, Trussell LO. Axon initial segment $\mathrm{Ca}^{2+}$ channels influence action potential generation and timing. Neuron 61: 259 271, 2009.

47. Benoit E, Dubois JM. Properties of maintained sodium current induced by a toxin from Androctonus scorpion in frog node of Ranvier. J Physiol 383: 93-114, 1987.

48. Berbel P, Innocenti GM. The development of the corpus callosum in cats: a light- and electron-microscopic study. J Comp Neurol 276: $132-156,1988$

49. Berghs S, Aggujaro D, Dirkx R Jr, Maksimova E, Stabach P, Hermel JM, Zhang JP, Philbrick W, Slepnev V, Ort T, Solimena M. betaIV spectrin, a new spectrin localized at axon initial 
segments and nodes of ranvier in the central and peripheral nervous system. J Cell Biol 151: 985-1002, 2000.

50. Bernard C, Anderson A, Becker A, Poolos NP, Beck H, Johnston D. Acquired dendritic channelopathy in temporal lobe epilepsy. Science 305: 532-535, 2004.

51. Bettler B, Kaupmann K, Mosbacher J, Gassmann M. Molecular structure and physiological functions of $\mathrm{GABA}(\mathrm{B})$ receptors. Physiol Rev 84: 835-867, 2004.

52. Bhattacharjee A, Gan L, Kaczmarek LK. Localization of the Slack potassium channel in the rat central nervous system. J Comp Neurol 454: 241-254, 2002.

53. Bi GQ, Poo MM. Synaptic modifications in cultured hippocampal neurons: dependence on spike timing, synaptic strength, and postsynaptic cell type. J Neurosci 18: 10464-10472, 1998.

54. Bidoret C, Ayon A, Barbour B, Casado M. Presynaptic NR2Acontaining NMDA receptors implement a high-pass filter synaptic plasticity rule. Proc Natl Acad Sci USA 106: 14126-14131, 2009.

55. Bielefeldt K, Jackson MB. A calcium-activated potassium channel causes frequency-dependent action-potential failures in a mammalian nerve terminal. J Neurophysiol 70: 284-298, 1993.

56. Biermann B, Ivankova-Susankova K, Bradaia A, Abdel Aziz S, Besseyrias V, Kapfhammer JP, Missler M, Gassmann M, Bettler B. The Sushi domains of $\mathrm{GABA}_{\mathrm{B}}$ receptors function as axonal targeting signals. J Neurosci 30: 1385-1394, 2010.

57. Binczak S, Eilbeck JC, Scott A. Ephaptic coupling of myelinated nerve fibers. Physica D 148: 159-174, 2001.

58. Bischofberger J, Engel D, Li L, Geiger JR, Jonas P. Patchclamp recording from mossy fiber terminals in hippocampal slices. Nat Protoc 1: 2075-2081, 2006.

59. Bischofberger J, Geiger JR, Jonas P. Timing and efficacy of $\mathrm{Ca}^{2+}$ channel activation in hippocampal mossy fiber boutons. $J$ Neurosci 22: 10593-10602, 2002.

60. Bischofberger J, Jonas P. Action potential propagation into the presynaptic dendrites of rat mitral cells. J Physiol 504: 359-365, 1997.

61. Blackstad TW, Kjaerheim A. Special axo-dendritic synapses in the hippocampal cortex: electron and light microscopic studies on the layer of mossy fibers. J Comp Neurol 117: 133-159, 1961.

62. Blinder KJ, Pumplin DW, Paul DL, Keller A. Intercellular interactions in the mammalian olfactory nerve. J Comp Neurol 466: 230-239, 2003.

63. Bliss TV, Lomo T. Long-lasting potentiation of synaptic transmission in the dentate area of the anaesthetized rabbit following stimulation of the perforant path. J Physiol 232: 331-356, 1973.

64. Blumenfeld H, Lampert A, Klein JP, Mission J, Chen MC, Rivera M, Dib-Hajj S, Brennan AR, Hains BC, Waxman SG. Role of hippocampal sodium channel Nav1.6 in kindling epileptogenesis. Epilepsia 50: 44-55, 2009.

65. Bobik M, Ellisman MH, Rudy B, Martone ME. Potassium channel subunit Kv3.2 and the water channel aquaporin-4 are selectively localized to cerebellar pinceau. Brain Res 1026: 168-178, 2004.

66. Boiko T, Rasband MN, Levinson SR, Caldwell JH, Mandel G, Trimmer JS, Matthews G. Compact myelin dictates the differential targeting of two sodium channel isoforms in the same axon. Neuron 30: 91-104, 2001.

67. Boiko T, Van Wart A, Caldwell JH, Levinson SR, Trimmer JS, Matthews G. Functional specialization of the axon initial segment by isoform-specific sodium channel targeting. J Neurosci 23: 23062313, 2003.

68. Bokil H, Laaris N, Blinder K, Ennis M, Keller A. Ephaptic interactions in the mammalian olfactory system. J Neurosci 21: RC173, 2001.

69. Bostock H, Sears TA. The internodal axon membrane: electrical excitability and continuous conduction in segmental demyelination. J Physiol 280: 273-301, 1978.

70. Boudkkazi S, Carlier E, Ankri N, Caillard O, Giraud P, Fronzaroli-Molinieres L, Debanne D. Release-dependent variations in synaptic latency: a putative code for short- and long-term synaptic dynamics. Neuron 56: 1048-1060, 2007.

71. Boudkkazi S, Fronzaroli-Molinieres L, Debanne D. Presynaptic action potential waveform determines cortical synaptic latency. J Physiol. 589: 1117-1131, 2011.
72. Bourque CW. Intraterminal recordings from the rat neurohypophysis in vitro. J Physiol 421: 247-262, 1990.

73. Brackenbury WJ, Calhoun JD, Chen C, Miyazaki H, Nukina N, Oyama F, Ranscht B, Isom LL. Functional reciprocity between $\mathrm{Na}^{+}$channel Nav1.6 and beta1 subunits in the coordinated regulation of excitability and neurite outgrowth. Proc Natl Acad Sci USA 107: 2283-2288, 2010.

74. Bradley J, Luo R, Otis TS, DiGregorio DA. Submillisecond optical reporting of membrane potential in situ using a neuronal tracer dye. J Neurosci 29: 9197-9209, 2009.

75. Brechet A, Fache MP, Brachet A, Ferracci G, Baude A, Irondelle M, Pereira S, Leterrier C, Dargent B. Protein kinase CK2 contributes to the organization of sodium channels in axonal membranes by regulating their interactions with ankyrin G. $J$ Cell Biol 183: 1101-1114, 2008.

76. Brigant JL, Mallart A. Presynaptic currents in mouse motor endings. J Physiol 333: 619-636, 1982.

77. Brock LG, Coombs JS, Eccles JC. The recording of potentials from motoneurones with an intracellular electrode. J Physiol 117: 431-460, 1952.

78. Brody DL, Yue DT. Release-independent short-term synaptic depression in cultured hippocampal neurons. $J$ Neurosci 20: 2480 2494, 2000.

79. Brown DA, Marsh S. Axonal GABA-receptors in mammalian peripheral nerve trunks. Brain Res 156: 187-191, 1978.

80. Browne DL, Gancher ST, Nutt JG, Brunt ER, Smith EA, Kramer P, Litt M. Episodic ataxia/myokymia syndrome is associated with point mutations in the human potassium channel gene, KCNA1. Nat Genet 8: 136-140, 1994

81. Brunig I, Scotti E, Sidler C, Fritschy JM. Intact sorting, targeting, and clustering of gamma-aminobutyric acid A receptor subtypes in hippocampal neurons in vitro. J Comp Neurol 443: 43-55, 2002.

82. Bucher D, Thirumalai V, Marder E. Axonal dopamine receptors activate peripheral spike initiation in a stomatogastric motor neuron. J Neurosci 23: 6866-6875, 2003.

83. Budd JM, Kovacs K, Ferecsko AS, Buzas P, Eysel UT, Kisvarday ZF. Neocortical axon arbors trade-off material and conduction delay conservation. PLoS Comput Biol 6: e1000711, 2010.

84. Burnstock G. Physiology and pathophysiology of purinergic neurotransmission. Physiol Rev 87: 659-797, 2007.

85. Burnstock G. Purinergic signalling and disorders of the central nervous system. Nat Rev Drug Discov 7: 575-590, 2008.

86. Burrows M, Siegler MV. Graded synaptic transmission between local interneurones and motor neurones in the metathoracic ganglion of the locust. J Physiol 285: 231-255, 1978.

87. Bush P, Sejnowski T. Inhibition synchronizes sparsely connected cortical neurons within and between columns in realistic network models. J Comput Neurosci 3: 91-110, 1996.

88. Caldwell JH, Schaller KL, Lasher RS, Peles E, Levinson SR. Sodium channel $\mathrm{Na}(\mathrm{v}) 1.6$ is localized at nodes of ranvier, dendrites, and synapses. Proc Natl Acad Sci USA 97: 5616-5620, 2000.

89. Caminos E, Garcia-Pino E, Martinez-Galan JR, Juiz JM. The potassium channel KCNQ5/Kv7.5 is localized in synaptic endings of auditory brainstem nuclei of the rat. J Comp Neurol 505: 363-378, 2007.

90. Campanac E, Daoudal G, Ankri N, Debanne D. Downregulation of dendritic $I(\mathrm{~h})$ in CA1 pyramidal neurons after LTP. J Neurosci 28: 8635-8643, 2008.

91. Campanac E, Debanne D. Spike timing-dependent plasticity: a learning rule for dendritic integration in rat CA1 pyramidal neurons. J Physiol 586: 779-793, 2008.

92. Cantrell AR, Catterall WA. Neuromodulation of $\mathrm{Na}^{+}$channels: an unexpected form of cellular plasticity. Nat Rev Neurosci 2: 397-407, 2001.

93. Canu MH, Carnaud M, Picquet F, Goutebroze L. Activity-dependent regulation of myelin maintenance in the adult rat. Brain Res 1252: 45-51, 2009.

94. Carlier E, Sourdet V, Boudkkazi S, Deglise P, Ankri N, Fronzaroli-Molinieres L, Debanne D. Metabotropic glutamate receptor subtype 1 regulates sodium currents in rat neocortical pyramidal neurons. J Physiol 577: 141-154, 2006. 
95. Carr CE, Konishi M. Axonal delay lines for time measurement in the owl's brainstem. Proc Natl Acad Sci USA 85: 8311-8315, 1988.

96. Carr CE, Konishi M. A circuit for detection of interaural time differences in the brain stem of the barn owl. J Neurosci 10: 3227-3246, 1990.

97. Casado M, Dieudonne S, Ascher P. Presynaptic N-methyl-Daspartate receptors at the parallel fiber-Purkinje cell synapse. Proc Natl Acad Sci USA 97: 11593-11597, 2000.

98. Casassus G, Blanchet C, Mulle C. Short-term regulation of information processing at the corticoaccumbens synapse. J Neurosci 25: 11504-11512, 2005.

99. Castelli L, Biella G, Toselli M, Magistretti J. Resurgent $\mathrm{Na}^{+}$ current in pyramidal neurones of rat perirhinal cortex: axonal location of channels and contribution to depolarizing drive during repetitive firing. J Physiol 582: 1179-1193, 2007.

100. Cattaert D, El Manira A. Shunting versus inactivation: analysis of presynaptic inhibitory mechanisms in primary afferents of the crayfish. J Neurosci 19: 6079-6089, 1999.

101. Cattaert D, el Manira A, Clarac F. Direct evidence for presynaptic inhibitory mechanisms in crayfish sensory afferents. J Neurophysiol 67: 610-624, 1992.

102. Cattaert D, Libersat F, El Manira AA. Presynaptic inhibition and antidromic spikes in primary afferents of the crayfish: a computational and experimental analysis. J Neurosci 21: 1007-1021, 2001.

103. Cestele S, Scalmani P, Rusconi R, Terragni B, Franceschetti S, Mantegazza M. Self-limited hyperexcitability: functional effect of a familial hemiplegic migraine mutation of the Nav1.1 (SCN1A) $\mathrm{Na}^{+}$channel. J Neurosci 28: 7273-7283, 2008.

104. Chance PF, Alderson MK, Leppig KA, Lensch MW, Matsunami N, Smith B, Swanson PD, Odelberg SJ, Disteche CM, Bird TD. DNA deletion associated with hereditary neuropathy with liability to pressure palsies. Cell 72: 143-151, 1993.

105. Chang SY, Zagha E, Kwon ES, Ozaita A, Bobik M, Martone ME, Ellisman MH, Heintz N, Rudy B. B. Distribution of Kv3.3 potassium channel subunits in distinct neuronal populations of mouse brain. J Comp Neurol 502: 953-972, 2007.

106. Chapman RA. Dependence on temperature of the conduction velocity of the action potential of the squid giant axon. Nature 213: 1143-1144, 1967.

107. Chen WR, Midtgaard J, Shepherd GM. Forward and backward propagation of dendritic impulses and their synaptic control in mitral cells. Science 278: 463-467, 1997.

108. Chen WR, Shen GY, Shepherd GM, Hines ML, Midtgaard J. Multiple modes of action potential initiation and propagation in mitral cell primary dendrite. J Neurophysiol 88: 2755-2764, 2002.

109. Chow A, Erisir A, Farb C, Nadal MS, Ozaita A, Lau D, Welker E, Rudy B. $\mathrm{K}^{+}$channel expression distinguishes subpopulations of parvalbumin- and somatostatin-containing neocortical interneurons. J Neurosci 19: 9332-9345, 1999.

110. Christie JM, Chiu DN, Jahr CE. $\mathrm{Ca}^{2+}$-dependent enhancement of release by subthreshold somatic depolarization. Nat Neurosci 14: 62-68, 2011.

111. Christie JM, Jahr CE. Dendritic NMDA receptors activate axonal calcium channels. Neuron 60: 298-307, 2008.

112. Christie JM, Jahr CE. Selective expression of ligand-gated ion channels in L5 pyramidal cell axons. J Neurosci 29: 11441-11450, 2009.

113. Chung SH, Raymond SA, Lettvin JY. Multiple meaning in single visual units. Brain Behav Evol 3: 72-101, 1970.

114. Claes L, Del-Favero J, Ceulemans B, Lagae L, Van Broeckhoven $\mathbf{C}$, De Jonghe $\mathbf{P}$. De novo mutations in the sodium-channel gene SCN1A cause severe myoclonic epilepsy of infancy. Am J Hum Genet 68: 1327-1332, 2001.

115. Clark B, Hausser M. Neural coding: hybrid analog and digital signalling in axons. Curr Biol 16: R585-588, 2006.

116. Clark BA, Monsivais P, Branco T, London M, Hausser M. The site of action potential initiation in cerebellar Purkinje neurons. Nat Neurosci 8: 137-139, 2005.

117. Clark BD, Goldberg EM, Rudy B. Electrogenic tuning of the axon initial segment. Neuroscientist 15: 651-668, 2009.
118. Cobb SR, Buhl EH, Halasy K, Paulsen O, Somogyi P. Synchronization of neuronal activity in hippocampus by individual GABAergic interneurons. Nature 378: 75-78, 1995.

119. Colbert CM, Johnston D. Axonal action-potential initiation and $\mathrm{Na}^{+}$channel densities in the soma and axon initial segment of subicular pyramidal neurons. J Neurosci 16: 6676-6686, 1996.

120. Colbert CM, Pan E. Ion channel properties underlying axonal action potential initiation in pyramidal neurons. Nat Neurosci 5: 533-538, 2002.

121. Coleman M. Axon degeneration mechanisms: commonality amid diversity. Nat Rev Neurosci 6: 889-898, 2005.

122. Colquhoun D, Ritchie JM. The interaction at equilibrium between tetrodotoxin and mammalian non-myelinated nerve fibres. $J$ Physiol 221: 533-553, 1972.

123. Constantino US, Fern R. Conduction block and glial injury induced in developing central white matter by glycine, GABA, noradrenalin, or nicotine, studied in isolated neonatal rat optic nerve. Glia 57: 1168-1177, 2009.

124. Coombs JS, Curtis DR, Eccles JC. The interpretation of spike potentials of motoneurones. J Physiol 139: 198-231, 1957.

125. Cooper EC, Harrington E, Jan YN, Jan LY. M channel KCNQ2 subunits are localized to key sites for control of neuronal network oscillations and synchronization in mouse brain. J Neurosci 21: 9529-9540, 2001.

126. Cooper EC, Milroy A, Jan YN, Jan LY, Lowenstein DH. Presynaptic localization of Kv1.4-containing A-type potassium channels near excitatory synapses in the hippocampus. J Neurosci 18 : 965-974, 1998.

127. Corlew R, Wang Y, Ghermazien H, Erisir A, Philpot BD. Developmental switch in the contribution of presynaptic and postsynaptic NMDA receptors to long-term depression. J Neurosci 27: 9835-9845, 2007.

128. Cox CL, Denk W, Tank DW, Svoboda K. Action potentials reliably invade axonal arbors of rat neocortical neurons. Proc Natl Acad Sci USA 97: 9724-9728, 2000.

129. Cox JJ, Reimann F, Nicholas AK, Thornton G, Roberts E, Springell K, Karbani G, Jafri H, Mannan J, Raashid Y, AlGazali L, Hamamy H, Valente EM, Gorman S, Williams R, McHale DP, Wood JN, Gribble FM, Woods CG. An SCN9A channelopathy causes congenital inability to experience pain. $\mathrm{Na}$ ture 444: 894-898, 2006.

130. Cregg R, Momin A, Rugiero F, Wood JN, Zhao J. Pain channelopathies. J Physiol 588: 1897-1904, 2010.

131. Cudmore RH, Fronzaroli-Molinieres L, Giraud P, Debanne D. Spike-time precision and network synchrony are controlled by the homeostatic regulation of the D-type potassium current. J Neurosci 30: 12885-12895, 2010.

132. Curtis DR, Lodge D. The depolarization of feline ventral horn group Ia spinal afferent terminations by GABA. Exp Brain Res 46: 215-233, 1982.

133. Cuttle MF, Rusznak Z, Wong AY, Owens S, Forsythe ID. Modulation of a presynaptic hyperpolarization-activated cationic current $[I(\mathrm{~h})]$ at an excitatory synaptic terminal in the rat auditory brainstem. J Physiol 534: 733-744, 2001.

134. Dallas ML, Atkinson L, Milligan CJ, Morris NP, Lewis DI, Deuchars SA, Deuchars J. Localization and function of the Kv3.1b subunit in the rat medulla oblongata: focus on the nucleus tractus solitarii. J Physiol 562: 655-672, 2005.

135. Daoudal G, Debanne D. Long-term plasticity of intrinsic excitability: learning rules and mechanisms. Learn Mem 10: 456-465, 2003.

136. Daoudal G, Hanada Y, Debanne D. Bidirectional plasticity of excitatory postsynaptic potential (EPSP)-spike coupling in CA1 hippocampal pyramidal neurons. Proc Natl Acad Sci USA 99: 14512-14517, 2002.

137. De Paola V, Holtmaat A, Knott G, Song S, Wilbrecht L, Caroni $\mathbf{P}$, Svoboda K. Cell type-specific structural plasticity of axonal branches and boutons in the adult neocortex. Neuron 49: 861-875, 2006.

138. De Vos KJ, Grierson AJ, Ackerley S, Miller CC. Role of axonal transport in neurodegenerative diseases. Annu Rev Neurosci 31: 151-173, 2008. 
139. Debanne D. Information processing in the axon. Nat Rev Neurosci 5: 304-316, 2004.

140. Debanne D, Boudkkazi S, Campanac E, Cudmore RH, Giraud P, Fronzaroli-Molinieres L, Carlier E, Caillard O. Paired-recordings from synaptically coupled cortical and hippocampal neurons in acute and cultured brain slices. Nat Protoc 3: 1559-1568, 2008.

141. Debanne D, Daoudal G, Sourdet V, Russier M. Brain plasticity and ion channels. J Physiol 97: 403-414, 2003.

142. Debanne D, Gahwiler BH, Thompson SM. Asynchronous preand postsynaptic activity induces associative long-term depression in area CA1 of the rat hippocampus in vitro. Proc Natl Acad Sci USA 91: 1148-1152, 1994.

143. Debanne D, Gahwiler BH, Thompson SM. Long-term synaptic plasticity between pairs of individual CA3 pyramidal cells in rat hippocampal slice cultures. J Physiol 507: 237-247, 1998.

144. Debanne D, Guerineau NC, Gahwiler BH, Thompson SM. Action-potential propagation gated by an axonal $I(\mathrm{~A})$-like $\mathrm{K}^{+}$conductance in hippocampus. Nature 389: 286-289, 1997.

145. Debanne D, Kopysova IL, Bras H, Ferrand N. Gating of action potential propagation by an axonal A-like potassium conductance in the hippocampus: a new type of non-synaptic plasticity. $J$ Physiol 93: 285-296, 1999.

146. Debanne D, Thompson SM, Gahwiler BH. A brief period of epileptiform activity strengthens excitatory synapses in the rat hippocampus in vitro. Epilepsia 47: 247-256, 2006.

147. Deiters OFK (Editor). Untersuchungen über Gehirn und Rückenmark des Menschen und der Säugethiere. Braunschweig: Vieweg, 1865.

148. Del Castillo J, Moore JW. On increasing the velocity of a nerve impulse. J Physiol 148: 665-670, 1959

149. Demerens C, Stankoff B, Logak M, Anglade P, Allinquant B, Couraud F, Zalc B, Lubetzki C. Induction of myelination in the central nervous system by electrical activity. Proc Natl Acad Sci USA 93: 9887-9892, 1996.

150. Desai NS, Rutherford LC, Turrigiano GG. Plasticity in the intrinsic excitability of cortical pyramidal neurons. Nat Neurosci 2 : 515-520, 1999.

151. Deschenes M, Landry P. Axonal branch diameter and spacing of nodes in the terminal arborization of identified thalamic and cortical neurons. Brain Res 191: 538-544, 1980.

152. Devaux J, Alcaraz G, Grinspan J, Bennett V, Joho R, Crest M, Scherer SS. Kv3.1b is a novel component of CNS nodes. $J$ Neurosci 23: 4509-4518, 2003.

153. Devaux J, Gow A. Tight junctions potentiate the insulative properties of small CNS myelinated axons. J Cell Biol 183: 909-921, 2008.

154. Devaux JJ, Kleopa KA, Cooper EC, Scherer SS. KCNQ2 is a nodal $\mathrm{K}^{+}$channel. J Neurosci 24: 1236-1244, 2004.

155. Diaz-Hernandez M, del Puerto A, Diaz-Hernandez JI, DiezZaera M, Lucas JJ, Garrido JJ, Miras-Portugal MT. Inhibition of the ATP-gated P2X7 receptor promotes axonal growth and branching in cultured hippocampal neurons. J Cell Sci 121: 3717$3728,2008$.

156. Dickson BJ. Molecular mechanisms of axon guidance. Science 298: 1959-1964, 2002.

157. Dodge FA, Cooley JW. Action potential of the motoneuron. IBM J Res Dev 17: 219-229, 1973.

158. Dodson PD, Billups B, Rusznak Z, Szucs G, Barker MC, Forsythe ID. Presynaptic rat Kv1.2 channels suppress synaptic terminal hyperexcitability following action potential invasion. $J$ Physiol 550: 27-33, 2003.

159. Dodson PD, Forsythe ID. Presynaptic $\mathrm{K}^{+}$channels: electrifying regulators of synaptic terminal excitability. Trends Neurosci 27 : 210-217, 2004

160. Dombeck DA, Blanchard-Desce M, Webb WW. Optical recording of action potentials with second-harmonic generation microscopy. J Neurosci 24: 999-1003, 2004.

161. Dotti CG, Sullivan CA, Banker GA. The establishment of polarity by hippocampal neurons in culture. J Neurosci 8: 1454-1468, 1988.
162. Draguhn A, Traub RD, Schmitz D, Jefferys JG. Electrical coupling underlies high-frequency oscillations in the hippocampus in vitro. Nature 394: 189-192, 1998.

163. Dreyer F, Penner R. The actions of presynaptic snake toxins on membrane currents of mouse motor nerve terminals. J Physiol 386: 455-463, 1987.

164. Du W, Bautista JF, Yang H, Diez-Sampedro A, You SA, Wang L, Kotagal P, Luders HO, Shi J, Cui J, Richerson GB, Wang QK. Calcium-sensitive potassium channelopathy in human epilepsy and paroxysmal movement disorder. Nat Genet 37: 733-738, 2005.

165. Dubois JM. Evidence for the existence of three types of potassium channels in the frog Ranvier node membrane. J Physiol 318: 297316, 1981

166. Dubois JM, Bergman C. Late sodium current in the node of Ranvier. Pflügers Arch 357: 145-148, 1975.

167. Ducreux C, Reynaud JC, Puizillout JJ. Spike conduction properties of T-shaped $\mathrm{C}$ neurons in the rabbit nodose ganglion. Pflügers Arch 424: 238-244, 1993.

168. Dudel J, Kuffler SW. Presynaptic inhibition at the crayfish neuromuscular junction. J Physiol 155: 543-562, 1961.

169. Duflocq A, Le Bras B, Bullier E, Couraud F, Davenne M. Nav1.1 is predominantly expressed in nodes of Ranvier and axon initial segments. Mol Cell Neurosci 39: 180-192, 2008.

170. Duguid IC, Smart TG. Retrograde activation of presynaptic NMDA receptors enhances GABA release at cerebellar interneuron-Purkinje cell synapses. Nat Neurosci 7: 525-533, 2004.

171. Dulla CG, Huguenard JR. Who let the spikes out? Nat Neurosci 12: 959-960, 2009

172. Dyball RE, Grossmann R, Leng G, Shibuki K. Spike propagation and conduction failure in the rat neural lobe. J Physiol 401: 241256,1988 .

173. Dzhashiashvili Y, Zhang Y, Galinska J, Lam I, Grumet M, Salzer JL. Nodes of Ranvier and axon initial segments are ankyrin G-dependent domains that assemble by distinct mechanisms. J Cell Biol 177: 857-870, 2007.

174. Eccles JC, Eccles RM, Magni F. Central inhibitory action attributable to presynaptic depolarization produced by muscle afferent volleys. J Physiol 159: 147-166, 1961.

175. Eccles JC, Schmidt R, Willis WD. Pharmacological studies on presynaptic inhibition. J Physiol 168: 500-530, 1963.

176. Edwards C, Ottoson D. The site of impulse initiation in a nerve cell of a crustacean stretch receptor. J Physiol 143: 138-148, 1958.

177. Elezgarai I, Diez J, Puente N, Azkue JJ, Benitez R, Bilbao A, Knopfel T, Donate-Oliver F, Grandes P. Subcellular localization of the voltage-dependent potassium channel Kv3.1b in postnatal and adult rat medial nucleus of the trapezoid body. Neuroscience 118: 889-898, 2003

178. Eng DL, Kocsis JD. Activity-dependent changes in extracellular potassium and excitability in turtle olfactory nerve. J Neurophysiol 57: 740-754, 1987

179. Engel D, Jonas P. Presynaptic action potential amplification by voltage-gated $\mathrm{Na}^{+}$channels in hippocampal mossy fiber boutons. Neuron 45: 405-417, 2005.

180. Engelman HS, MacDermott AB. Presynaptic ionotropic receptors and control of transmitter release. Nat Rev Neurosci 5: 135$145,2004$.

181. Epsztein J, Lee AK, Chorev E, Brecht M. Impact of spikelets on hippocampal CA1 pyramidal cell activity during spatial exploration. Science 327: 474-477, 2010.

182. Escayg A, Heils A, MacDonald BT, Haug K, Sander T, Meisler MH. A novel SCN1A mutation associated with generalized epilepsy with febrile seizures plus-and prevalence of variants in patients with epilepsy. Am J Hum Genet 68: 866-873, 2001.

183. Eshed Y, Feinberg K, Carey DJ, Peles E. Secreted gliomedin is a perinodal matrix component of peripheral nerves. J Cell Biol 177: 551-562, 2007.

184. Evans CG, Jing J, Rosen SC, Cropper EC. Regulation of spike initiation and propagation in an Aplysia sensory neuron: gating-in via central depolarization. J Neurosci 23: 2920-2931, 2003.

185. Evans CG, Ludwar B, Cropper EC. Mechanoafferent neuron with an inexcitable somatic region: consequences for the regula- 
tion of spike propagation and afferent transmission. $J$ Neurophysiol 97: 3126-3130, 2007.

186. Faisal AA, Laughlin SB. Stochastic simulations on the reliability of action potential propagation in thin axons. PLoS Comput Biol 3: e79, 2007.

187. Fatt P. Sequence of events in synaptic activation of a motoneurone. J Neurophysiol 20: 61-80, 1957.

188. Fernandez-Alacid L, Aguado C, Ciruela F, Martin R, Colon J, Cabanero MJ, Gassmann M, Watanabe M, Shigemoto R, Wickman K, Bettler B, Sanchez-Prieto J, Lujan R. Subcellular compartment-specific molecular diversity of pre- and post-synaptic GABA-activated GIRK channels in Purkinje cells. J Neurochem 110: 1363-1376, 2009.

189. Fertleman CR, Baker MD, Parker KA, Moffatt S, Elmslie FV, Abrahamsen B, Ostman J, Klugbauer N, Wood JN, Gardiner RM, Rees M. SCN9A mutations in paroxysmal extreme pain disorder: allelic variants underlie distinct channel defects and phenotypes. Neuron 52: 767-774, 2006.

190. Fields RD. Oligodendrocytes changing the rules: action potentials in glia and oligodendrocytes controlling action potentials. Neuroscientist 14: 540-543, 2008.

191. Fields RD, Ni Y. Nonsynaptic communication through ATP release from volume-activated anion channels in axons. Sci Signal 3: ra73, 2010.

192. Finnegan TF, Chen SR, Pan HL. Mu opioid receptor activation inhibits GABAergic inputs to basolateral amygdala neurons through Kv1.1/1.2 channels. J Neurophysiol 95: 2032-2041, 2006.

193. Fleidervish IA, Lasser-Ross N, Gutnick MJ, Ross WN. $\mathrm{Na}^{+}$ imaging reveals little difference in action potential-evoked $\mathrm{Na}^{+}$ influx between axon and soma. Nat Neurosci 2010.

194. Forti L, Pouzat C, Llano I. Action potential-evoked $\mathrm{Ca}^{2+}$ signals and calcium channels in axons of developing rat cerebellar interneurones. J Physiol 527: 33-48, 2000.

195. Foster RE, Whalen CC, Waxman SG. Reorganization of the axon membrane in demyelinated peripheral nerve fibers: morphological evidence. Science 210: 661-663, 1980.

196. Foust A, Popovic M, Zecevic D, McCormick DA. Action potentials initiate in the axon initial segment and propagate through axon collaterals reliably in cerebellar Purkinje neurons. J Neurosci 30: 6891-6902, 2010.

197. Frank K, Fuortes MGF. Presynaptic and postsynaptic inhibtion of monosynaptic reflexes. Federation Proc 16: 39-40, 1957.

198. Franz DN, Iggo A. Conduction failure in myelinated and nonmyelinated axons at low temperatures. J Physiol 199: 319-345, 1968.

199. Frick A, Magee J, Johnston D. LTP is accompanied by an enhanced local excitability of pyramidal neuron dendrites. Nat Neurosci 7: 126-135, 2004.

200. Fricker D, Miles R. EPSP amplification and the precision of spike timing in hippocampal neurons. Neuron 28: 559-569, 2000.

201. Fricker D, Verheugen JA, Miles R. Cell-attached measurements of the firing threshold of rat hippocampal neurones. J Physiol 517: 791-804, 1999 .

202. Fuortes MG, Frank K, Becker MC. Steps in the production of motoneuron spikes. J Gen Physiol 40: 735-752, 1957.

203. Galimberti I, Bednarek E, Donato F, Caroni P. EphA4 signaling in juveniles establishes topographic specificity of structural plasticity in the hippocampus. Neuron 65: 627-642, 2010.

204. Galimberti I, Gogolla N, Alberi S, Santos AF, Muller D, Caroni $\mathbf{P}$. Long-term rearrangements of hippocampal mossy fiber terminal connectivity in the adult regulated by experience. Neuron 50: 749-763, 2006

205. Ganguly K, Kiss L, Poo M. Enhancement of presynaptic neuronal excitability by correlated presynaptic and postsynaptic spiking. Nat Neurosci 3: 1018-1026, 2000.

206. Garrido JJ, Giraud P, Carlier E, Fernandes F, Moussif A, Fache MP, Debanne D, Dargent B. A targeting motif involved in sodium channel clustering at the axonal initial segment. Science 300: 2091-2094, 2003.

207. Gastrein P, Campanac E, Gasselin C, Cudmore RH, Bialowas A, Carlier E, Fronzaroli-Molinieres L, Ankri N, Debanne D. The role of hyperpolarization-activated cationic current $\left(I_{h}\right)$ in spike-time precision and intrinsic resonance in cortical neurons in vitro. $J$ Physiol. In press.

208. Geiger JR, Bischofberger J, Vida I, Frobe U, Pfitzinger S, Weber HJ, Haverkampf $\mathbf{K}$, Jonas $\mathbf{P}$. Patch-clamp recording in brain slices with improved slicer technology. Pflügers Arch 443: 491-501, 2002.

209. Geiger JR, Jonas P. Dynamic control of presynaptic $\mathrm{Ca}^{2+}$ inflow by fast-inactivating $\mathrm{K}^{+}$channels in hippocampal mossy fiber boutons. Neuron 28: 927-939, 2000.

210. Giuditta A, Chun JT, Eyman M, Cefaliello C, Bruno AP, Crispino M. Local gene expression in axons and nerve endings: the glia-neuron unit. Physiol Rev 88: 515-555, 2008.

211. Glickfeld LL, Roberts JD, Somogyi P, Scanziani M. Interneurons hyperpolarize pyramidal cells along their entire somatodendritic axis. Nat Neurosci 12: 21-23, 2009.

212. Glitsch M, Marty A. Presynaptic effects of NMDA in cerebellar Purkinje cells and interneurons. J Neurosci 19: 511-519, 1999.

213. Goaillard JM, Schulz DJ, Kilman VL, Marder E. Octopamine modulates the axons of modulatory projection neurons. J Neurosci 24: 7063-7073, 2004.

214. Gogan P, Gueritaud JP, Tyc-Dumont S. Comparison of antidromic and orthodromic action potentials of identified motor axons in the cat's brain stem. J Physiol 335: 205-220, 1983.

215. Gogan P, Schmiedel-Jakob I, Chitti Y, Tyc-Dumont S. Fluorescence imaging of local membrane electric fields during the excitation of single neurons in culture. Biophys J 69: 299-310, 1995.

216. Gogolla N, Galimberti I, Deguchi Y, Caroni P. Wnt signaling mediates experience-related regulation of synapse numbers and mossy fiber connectivities in the adult hippocampus. Neuron 62 : 510-525, 2009.

217. Goldberg EM, Clark BD, Zagha E, Nahmani M, Erisir A, Rudy B. $\mathrm{K}^{+}$channels at the axon initial segment dampen near-threshold excitability of neocortical fast-spiking GABAergic interneurons. Neuron 58: 387-400, 2008.

218. Goldberg EM, Watanabe S, Chang SY, Joho RH, Huang ZJ, Leonard CS, Rudy B. Specific functions of synaptically localized potassium channels in synaptic transmission at the neocortical GABAergic fast-spiking cell synapse. J Neurosci 25: 5230-5235, 2005.

219. Goldfinger MD. Computation of high safety factor impulse propagation at axonal branch points. Neuroreport 11: 449-456, 2000.

220. Golding NL, Spruston N. Dendritic sodium spikes are variable triggers of axonal action potentials in hippocampal CA1 pyramidal neurons. Neuron 21: 1189-1200, 1998.

221. Goldstein SS, Rall W. Changes of action potential shape and velocity for changing core conductor geometry. Biophys $J$ 14: 731-757, 1974.

222. Gomis-Ruth S, Wierenga CJ, Bradke F. Plasticity of polarization: changing dendrites into axons in neurons integrated in neuronal circuits. Curr Biol 18: 992-1000, 2008.

223. Gong B, Rhodes KJ, Bekele-Arcuri Z, Trimmer JS. Type I and type II $\mathrm{Na}^{+}$channel alpha-subunit polypeptides exhibit distinct spatial and temporal patterning, association with auxiliary subunits in rat brain. J Comp Neurol 412: 342-352, 1999.

224. Gossard JP, Cabelguen JM, Rossignol S. Intra-axonal recordings of cutaneous primary afferents during fictive locomotion in the cat. J Neurophysiol 62: 1177-1188, 1989.

225. Grafe P, Quasthoff S, Grosskreutz J, Alzheimer C. Function of the hyperpolarization-activated inward rectification in nonmyelinated peripheral rat and human axons. J Neurophysiol 77: 421-426, 1997.

226. Graham B, Redman S. A simulation of action potentials in synaptic boutons during presynaptic inhibition. J Neurophysiol 71: 538-549, 1994.

227. Graubard K, Raper JA, Hartline DK. Graded synaptic transmission between spiking neurons. Proc Natl Acad Sci USA 77: 3733$3735,1980$.

228. Grinvald A, Salzberg BM, Lev-Ram V, Hildesheim R. Optical recording of synaptic potentials from processes of single neurons using intracellular potentiometric dyes. Biophys $J$ 51: 643-651, 1987.

229. Grosse G, Draguhn A, Hohne L, Tapp R, Veh RW, AhnertHilger G. Expression of Kv1 potassium channels in mouse hip- 
pocampal primary cultures: development and activity-dependent regulation. J Neurosci 20: 1869-1882, 2000.

230. Grossman Y, Parnas I, Spira ME. Differential conduction block in branches of a bifurcating axon. J Physiol 295: 283-305, 1979.

231. Grossman Y, Parnas I, Spira ME. Mechanisms involved in differential conduction of potentials at high frequency in a branching axon. J Physiol 295: 307-322, 1979.

232. Grubb MS, Burrone J. Activity-dependent relocation of the axon initial segment fine-tunes neuronal excitability. Nature 465: 10701074, 2010.

233. Grubb MS, Burrone J. Building and maintaining the axon initial segment. Curr Opin Neurobiol 20: 481-488, 2010.

234. Gu XN. Effect of conduction block at axon bifurcations on synaptic transmission to different postsynaptic neurones in the leech. $J$ Physiol 441: 755-778, 1991.

235. Gulyas AI, Miles R, Hajos N, Freund TF. Precision and variability in postsynaptic target selection of inhibitory cells in the hippocampal CA3 region. Eur J Neurosci 5: 1729-1751, 1993.

236. Gutkin B, Ermentrout GB. Neuroscience: spikes too kinky in the cortex? Nature 440: 999-1000, 2006.

237. Hagiwara S, Tasaki I. A study on the mechanism of impulse transmission across the giant synapse of the squid. J Physiol 143: $114-137,1958$

238. Han C, Rush AM, Dib-Hajj SD, Li S, Xu Z, Wang Y, Tyrrell L, Wang X, Yang Y, Waxman SG. Sporadic onset of erythermalgia: a gain-of-function mutation in Nav1.7. Ann Neurol 59: 553-558, 2006.

239. Hata Y, Stryker MP. Control of thalamocortical afferent rearrangement by postsynaptic activity in developing visual cortex. Science 265: 1732-1735, 1994.

240. Hata Y, Tsumoto T, Stryker MP. Selective pruning of more active afferents when cat visual cortex is pharmacologically inhibited. Neuron 22: 375-381, 1999.

241. Hatt H, Smith DO. Synaptic depression related to presynaptic axon conduction block. J Physiol 259: 367-393, 1976.

242. Hausser M, Stuart G, Racca C, Sakmann B. Axonal initiation and active dendritic propagation of action potentials in substantia nigra neurons. Neuron 15: 637-647, 1995.

243. He Y, Zorumski CF, Mennerick S. Contribution of presynaptic $\mathrm{Na}^{+}$channel inactivation to paired-pulse synaptic depression in cultured hippocampal neurons. J Neurophysiol 87: 925-936, 2002.

244. Hedstrom KL, Ogawa Y, Rasband MN. AnkyrinG is required for maintenance of the axon initial segment and neuronal polarity. $J$ Cell Biol 183: 635-640, 2008.

245. Hedstrom KL, Xu X, Ogawa Y, Frischknecht R, Seidenbecher CI, Shrager P, Rasband MN. Neurofascin assembles a specialized extracellular matrix at the axon initial segment. J Cell Biol 178: 875-886, 2007.

246. Hee Kim J, Kushmerick C, von Gersdorff H. Presynaptic resurgent $\mathrm{Na}^{+}$currents sculpt the action potential waveform and increase firing reliability at a CNS nerve terminal. J Neurosci 30 : 15479-15490, 2010.

247. Heitler WJ, Goodman CS. Multiple sites of spike initiation in a bifurcating locust neurone. J Exp Biol 76: 63-84, 1978.

248. Henze DA, Buzsaki G. Action potential threshold of hippocampal pyramidal cells in vivo is increased by recent spiking activity. Neuroscience 105: 121-130, 2001.

249. Hestrin S, Galarreta M. Electrical synapses define networks of neocortical GABAergic neurons. Trends Neurosci 28: 304-309, 2005.

250. Hirokawa N, Noda Y. Intracellular transport and kinesin superfamily proteins, KIFs: structure, function, and dynamics. Physiol Rev 88: $1089-1118,2008$.

251. Hodgkin AL. A note on conduction velocity. J Physiol 125: 221$224,1954$.

252. Hodgkin AL. The relation between conduction velocity and the electrical resistance outside a nerve fibre. J Physiol 94: 560-570, 1939.

253. Hodgkin AL, Katz B. The effect of temperature on the electrical activity of the giant axon of the squid. J Physiol 109: 240-249, 1949.

253a.Hodgkin AL, Rushton WAH. The electrical constants of a crustacean nerve fibre. Proc R Soc Lond B Biol Sci 133: 444-449, 1946.

254. Holt GR, Koch C. Electrical interactions via the extracellular potential near cell bodies. J Comput Neurosci 6: 169-184, 1999.
255. Holtmaat A, Bonhoeffer T, Chow DK, Chuckowree J, De Paola V, Hofer SB, Hubener M, Keck T, Knott G, Lee WC, Mostany R, Mrsic-Flogel TD, Nedivi E, Portera-Cailliau C, Svoboda K, Trachtenberg JT, Wilbrecht L. Long-term, highresolution imaging in the mouse neocortex through a chronic cranial window. Nat Protoc 4: 1128-1144, 2009.

256. Holtmaat A, Svoboda K. Experience-dependent structural synaptic plasticity in the mammalian brain. Nat Rev Neurosci 10: 647658, 2009.

257. Horresh I, Poliak S, Grant S, Bredt D, Rasband MN, Peles E. Multiple molecular interactions determine the clustering of Caspr2 and Kv1 channels in myelinated axons. J Neurosci 28: 14213-14222, 2008.

258. Hu H, Shao LR, Chavoshy S, Gu N, Trieb M, Behrens R, Laake P, Pongs O, Knaus HG, Ottersen OP, Storm JF. Presynaptic $\mathrm{Ca}^{2+}$-activated $\mathrm{K}^{+}$channels in glutamatergic hippocampal terminals and their role in spike repolarization and regulation of transmitter release. J Neurosci 21: 9585-9597, 2001.

259. Hu W, Tian C, Li T, Yang M, Hou H, Shu Y. Distinct contributions of $\mathrm{Na}(\mathrm{v}) 1.6$ and $\mathrm{Na}(\mathrm{v}) 1.2$ in action potential initiation and backpropagation. Nat Neurosci 12: 996-1002, 2009.

260. Huang H, Trussell LO. Control of presynaptic function by a persistent $\mathrm{Na}^{+}$current. Neuron 60: 975-979, 2008.

261. Hubbard JI, Willis WD. The effects of depolarization of motor nerve terminals upon the release of transmitter by nerve impulses. J Physiol 194: 381-405, 1968.

262. Hubbard JI, Willis WD. Hyperpolarization of mammalian motor nerve terminals. J Physiol 163: 115-137, 1962.

263. Hugel S, Schlichter R. Presynaptic P2X receptors facilitate inhibitory GABAergic transmission between cultured rat spinal cord dorsal horn neurons. $J$ Neurosci 20: 2121-2130, 2000.

264. Hursch JB. Conduction velocity and diameter of nerve fibers. Am J Physiol 127: 131-139, 1939.

265. Huxley AF, Stampfli R. Evidence for saltatory conduction in peripheral myelinated nerve fibres. J Physiol 108: 315-339, 1949.

266. Ibarretxe G, Perrais D, Jaskolski F, Vimeney A, Mulle C. Fast regulation of axonal growth cone motility by electrical activity. $J$ Neurosci 27: 7684-7695, 2007.

267. Inda MC, DeFelipe J, Munoz A. Voltage-gated ion channels in the axon initial segment of human cortical pyramidal cells and their relationship with chandelier cells. Proc Natl Acad Sci USA 103: 2920-2925, 2006.

268. Ishikawa T, Nakamura Y, Saitoh N, Li WB, Iwasaki S, Takahashi T. Distinct roles of Kv1 and Kv3 potassium channels at the calyx of Held presynaptic terminal. J Neurosci 23: 10445-10453, 2003.

269. Ishizuka N, Weber J, Amaral DG. Organization of intrahippocampal projections originating from CA3 pyramidal cells in the rat. J Comp Neurol 295: 580-623, 1990.

270. Isope P, Franconville R, Barbour B, Ascher P. Repetitive firing of rat cerebellar parallel fibres after a single stimulation. $J$ Physiol 554: 829-839, 2004.

271. Izhikevich EM. Polychronization: computation with spikes. Neural Comput 18: 245-282, 2006.

272. Jackson MB, Zhang SJ. Action potential propagation and propagation block by GABA in rat posterior pituitary nerve terminals. $J$ Physiol 483: 597-611, 1995.

273. Jang IS, Nakamura M, Ito Y, Akaike N. Presynaptic GABA receptors facilitate spontaneous glutamate release from presynaptic terminals on mechanically dissociated rat CA3 pyramidal neurons. Neuroscience 138: 25-35, 2006.

274. Jo YH, Schlichter R. Synaptic corelease of ATP and GABA in cultured spinal neurons. Nat Neurosci 2: 241-245, 1999.

275. Johnston J, Griffin SJ, Baker C, Skrzypiec A, Chernova T, Forsythe ID. Initial segment Kv2.2 channels mediate a slow delayed rectifier and maintain high frequency action potential firing in medial nucleus of the trapezoid body neurons. $J$ Physiol 586: 3493-3509, 2008.

276. Kaplan MR, Meyer-Franke A, Lambert S, Bennett V, Duncan ID, Levinson SR, Barres BA. Induction of sodium channel clustering by oligodendrocytes. Nature 386: 724-728, 1997.

277. Katz B. The effect of electrolyte deficiency on the rate of conduction in a single nerve fibre. J Physiol 106: 411-417, 1947. 
278. Katz B, Miledi R. Propagation of electric activity in motor nerve terminals. Proc R Soc Lond B Biol Sci 161: 453-482, 1965.

279. Katz B, Miledi R. A study of synaptic transmission in the absence of nerve impulses. J Physiol 192: 407-436, 1967.

280. Katz B, Schmitt OH. Electric interaction between two adjacent nerve fibres. $J$ Physiol 97: 471-488, 1940.

281. Katz B, Schmitt OH. A note on interaction between nerve fibers. J Physiol 100: 369-371, 1942.

282. Kawai H, Lazar R, Metherate R. Nicotinic control of axon excitability regulates thalamocortical transmission. Nat Neurosci 10: 1168-1175, 2007.

283. Khaliq ZM, Raman IM. Axonal propagation of simple and complex spikes in cerebellar Purkinje neurons. J Neurosci 25: 454-463, 2005.

284. Khaliq ZM, Raman IM. Relative contributions of axonal and somatic Na channels to action potential initiation in cerebellar Purkinje neurons. J Neurosci 26: 1935-1944, 2006.

285. Khirug S, Yamada J, Afzalov R, Voipio J, Khiroug L, Kaila K. GABAergic depolarization of the axon initial segment in cortical principal neurons is caused by the $\mathrm{Na}-\mathrm{K}-2 \mathrm{Cl}$ cotransporter NKCC1. J Neurosci 28: 4635-4639, 2008.

286. Klausberger T, Magill PJ, Marton LF, Roberts JD, Cobden PM, Buzsaki G, Somogyi P. Brain-state- and cell-type-specific firing of hippocampal interneurons in vivo. Nature 421: 844-848, 2003.

287. Knaus HG, Schwarzer C, Koch RO, Eberhart A, Kaczorowski GJ, Glossmann H, Wunder F, Pongs O, Garcia ML, Sperk G. Distribution of high-conductance $\mathrm{Ca}^{2+}$-activated $\mathrm{K}^{+}$channels in rat brain: targeting to axons and nerve terminals. J Neurosci 16: 955963, 1996.

288. Kocsis JD, Ruiz JA, Cummins KL. Modulation of axonal excitability mediated by surround electric activity: an intra-axonal study. Exp Brain Res 47: 151-153, 1982.

289. Koester HJ, Sakmann B. Calcium dynamics associated with action potentials in single nerve terminals of pyramidal cells in layer 2/3 of the young rat neocortex. J Physiol 529: 625-646, 2000.

290. Kole MH, Ilschner SU, Kampa BM, Williams SR, Ruben PC, Stuart GJ. Action potential generation requires a high sodium channel density in the axon initial segment. Nat Neurosci 11: 178-186, 2008.

291. Kole MH, Letzkus JJ, Stuart GJ. Axon initial segment Kv1 channels control axonal action potential waveform and synaptic efficacy. Neuron 55: 633-647, 2007.

292. Kole MH, Stuart GJ. Is action potential threshold lowest in the axon? Nat Neurosci 11: 1253-1255, 2008.

293. Kölliker A. Neurologische Bemerkungen. Z Wiss Zool 1: 135-163, 1849 .

294. Konishi Y, Setou M. Tubulin tyrosination navigates the kinesin-1 motor domain to axons. Nat Neurosci 12: 559-567, 2009.

295. Kopysova IL, Debanne D. Critical role of axonal A-type $\mathrm{K}^{+}$ channels and axonal geometry in the gating of action potential propagation along CA3 pyramidal cell axons: a simulation study. $J$ Neurosci 18: 7436-7451, 1998.

296. Kordeli E, Lambert S, Bennett V. AnkyrinG: a new ankyrin gene with neural-specific isoforms localized at the axonal initial segment and node of Ranvier. J Biol Chem 270: 2352-2359, 1995.

297. Krarup C, Moldovan M. Nerve conduction and excitability studies in peripheral nerve disorders. Curr Opin Neurol 22: 460-466, 2009 .

298. Kress GJ, Dowling MJ, Eisenman LN, Mennerick S. Axonal sodium channel distribution shapes the depolarized action potential threshold of dentate granule neurons. Hippocampus 20: 558$571,2010$.

299. Kress GJ, Dowling MJ, Meeks JP, Mennerick S. High threshold, proximal initiation, and slow conduction velocity of action potentials in dentate granule neuron mossy fibers. $J$ Neurophysiol 100: 281-291, 2008.

300. Kress GJ, Mennerick S. Action potential initiation and propagation: upstream influences on neurotransmission. Neuroscience 158: 211-222, 2009.

301. Krnjevic K, Miledi R. Presynaptic failure of neuromuscular propagation in rats. J Physiol 149: 1-22, 1959.
302. Kuba H, Ishii TM, Ohmori H. Axonal site of spike initiation enhances auditory coincidence detection. Nature 444: 1069-1072, 2006.

303. Kuba H, Ohmori H. Roles of axonal sodium channels in precise auditory time coding at nucleus magnocellularis of the chick. $J$ Physiol 587: 87-100, 2009.

304. Kuba H, Oichi Y, Ohmori H. Presynaptic activity regulates $\mathrm{Na}^{+}$ channel distribution at the axon initial segment. Nature 465: 10751078, 2010.

305. Kues WA, Wunder F. Heterogeneous expression patterns of mammalian potassium channel genes in developing and adult rat brain. Eur J Neurosci 4: 1296-1308, 1992.

306. Kullmann DM. Neurological channelopathies. Annu Rev Neurosci 33: 151-172, 2010.

307. Kullmann DM, Ruiz A, Rusakov DM, Scott R, Semyanov A, Walker MC. Presynaptic, extrasynaptic and axonal $\mathrm{GABA}_{\mathrm{A}}$ receptors in the CNS: where and why? Prog Biophys Mol Biol 87: 33-46, 2005.

308. Ladera C, del Carmen Godino M, Jose Cabanero M, Torres M, Watanabe M, Lujan R, Sanchez-Prieto J. Pre-synaptic GABA receptors inhibit glutamate release through GIRK channels in rat cerebral cortex. J Neurochem 107: 1506-1517, 2008.

309. Lafleur J, Zytnicki D, Horcholle-Bossavit G, Jami L. Depolarization of Ib afferent axons in the cat spinal cord during homonymous muscle contraction. J Physiol 445: 345-354, 1992.

310. Lai HC, Jan LY. The distribution and targeting of neuronal voltage-gated ion channels. Nat Rev Neurosci 7: 548-562, 2006.

311. Lambe EK, Aghajanian GK. The role of Kv1.2-containing potassium channels in serotonin-induced glutamate release from thalamocortical terminals in rat frontal cortex. J Neurosci 21: 9955-9963, 2001.

312. Lamotte d'Incamps B, Destombes J, Thiesson D, Hellio R, Lasserre X, Kouchtir-Devanne N, Jami L, Zytnicki D. Indications for GABA-immunoreactive axo-axonic contacts on the intraspinal arborization of a Ib fiber in cat: a confocal microscope study. J Neurosci 18: 10030-10036, 1998.

313. Lamotte D'Incamps B, Meunier C, Monnet ML, Jami L, Zytnicki D. Reduction of presynaptic action potentials by PAD: model and experimental study. J Comput Neurosci 5: 141-156, 1998.

314. Lang PM, Burgstahler R, Sippel W, Irnich D, Schlotter-Weigel B, Grafe P. Characterization of neuronal nicotinic acetylcholine receptors in the membrane of unmyelinated human C-fiber axons by in vitro studies. J Neurophysiol 90: 3295-3303, 2003.

315. Lang PM, Fleckenstein J, Passmore GM, Brown DA, Grafe P. Retigabine reduces the excitability of unmyelinated peripheral human axons. Neuropharmacology 54: 1271-1278, 2008.

316. Lang PM, Moalem-Taylor G, Tracey DJ, Bostock H, Grafe P. Activity-dependent modulation of axonal excitability in unmyelinated peripheral rat nerve fibers by the $5-\mathrm{HT}_{3}$ serotonin receptor. $J$ Neurophysiol 96: 2963-2971, 2006.

317. Lapicque L. Recherches quantitatives sur l'excitation électrique des nerfs traitée comme une polarisation. J Physiol Pathol Générale 9: 622-635, 1907.

318. Larkum ME, Rioult MG, Luscher HR. Propagation of action potentials in the dendrites of neurons from rat spinal cord slice cultures. J Neurophysiol 75: 154-170, 1996.

319. Laube G, Roper J, Pitt JC, Sewing S, Kistner U, Garner CC, Pongs O, Veh RW. Ultrastructural localization of Shaker-related potassium channel subunits and synapse-associated protein 90 to septate-like junctions in rat cerebellar Pinceaux. Brain Res 42: 51-61, 1996.

320. Leao RM, Kushmerick C, Pinaud R, Renden R, Li GL, Taschenberger H, Spirou G, Levinson SR, von Gersdorff H. Presynaptic $\mathrm{Na}^{+}$channels: locus, development, and recovery from inactivation at a high-fidelity synapse. J Neurosci 25: 3724-3738, 2005.

321. Lee AK, Epsztein J, Brecht M. Head-anchored whole-cell recordings in freely moving rats. Nat Protoc 4: 385-392, 2009.

322. Lee AK, Manns ID, Sakmann B, Brecht M. Whole-cell recordings in freely moving rats. Neuron 51: 399-407, 2006.

323. Leveque C, el Far O, Martin-Moutot N, Sato K, Kato R, Takahashi M, Seagar MJ. Purification of the N-type calcium channel associated with syntaxin and synaptotagmin. A complex implicated in synaptic vesicle exocytosis. J Biol Chem 269: 6306-6312, 1994. 
324. Li CY, Lu JT, Wu CP, Duan SM, Poo MM. Bidirectional modification of presynaptic neuronal excitability accompanying spike timing-dependent synaptic plasticity. Neuron 41: 257-268, 2004.

325. Li XG, Somogyi P, Ylinen A, Buzsaki G. The hippocampal CA3 network: an in vivo intracellular labeling study. J Comp Neurol 339: 181-208, 1994.

326. Lillie RS. Factors affecting transmission and recovery in the passive iron nerve model. J Gen Physiol 7: 473-507, 1925.

327. Lin JW. Electrophysiological events recorded at presynaptic terminals of the crayfish neuromuscular junction with a voltage indicator. J Physiol 586: 4935-4950, 2008.

328. Lin JW, Faber DS. Modulation of synaptic delay during synaptic plasticity. Trends Neurosci 25: 449-455, 2002.

329. Lindgren CA, Moore JW. Identification of ionic currents at presynaptic nerve endings of the lizard. J Physiol 414: 201-222, 1989.

330. Llano I, Tan YP, Caputo C. Spatial heterogeneity of intracellular $\mathrm{Ca}^{2+}$ signals in axons of basket cells from rat cerebellar slices. $J$ Physiol 502: 509-519, 1997.

331. Llinas R, Sugimori M. Electrophysiological properties of in vitro Purkinje cell dendrites in mammalian cerebellar slices. J Physiol 305: 197-213, 1980

332. Lorincz A, Nusser Z. Cell-type-dependent molecular composition of the axon initial segment. J Neurosci 28: 14329-14340, 2008.

333. Lorincz A, Nusser Z. Molecular identity of dendritic voltage-gated sodium channels. Science 328: 906-909, 2010.

334. Lujan R, Albasanz JL, Shigemoto R, Juiz JM. Preferential localization of the hyperpolarization-activated cyclic nucleotidegated cation channel subunit HCN1 in basket cell terminals of the rat cerebellum. Eur J Neurosci 21: 2073-2082, 2005.

335. Luscher C, Streit J, Lipp P, Luscher HR. Action potential propagation through embryonic dorsal root ganglion cells in culture. II. Decrease of conduction reliability during repetitive stimulation. $J$ Neurophysiol 72: 634-643, 1994.

336. Luscher C, Streit J, Quadroni R, Luscher HR. Action potential propagation through embryonic dorsal root ganglion cells in culture. I. Influence of the cell morphology on propagation properties. J Neurophysiol 72: 622-633, 1994.

337. Luscher HR, Shiner JS. Computation of action potential propagation and presynaptic bouton activation in terminal arborizations of different geometries. Biophys J 58: 1377-1388, 1990.

338. Luscher HR, Shiner JS. Simulation of action potential propagation in complex terminal arborizations. Biophys J 58: 1389-1399, 1990.

339. Macagno ER, Muller KJ, Pitman RM. Conduction block silences parts of a chemical synapse in the leech central nervous system. $J$ Physiol 387: 649-664, 1987.

340. Maccaferri G, Roberts JD, Szucs P, Cottingham CA, Somogyi P. Cell surface domain specific postsynaptic currents evoked by identified GABAergic neurones in rat hippocampus in vitro. $J$ Physiol 524: 91-116, 2000.

341. Mackenzie PJ, Murphy TH. High safety factor for action potential conduction along axons but not dendrites of cultured hippocampal and cortical neurons. J Neurophysiol 80: 2089-2101, 1998.

342. Mackenzie PJ, Umemiya M, Murphy TH. $\mathrm{Ca}^{2+}$ imaging of CNS axons in culture indicates reliable coupling between single action potentials and distal functional release sites. Neuron 16: 783-795, 1996.

343. Madeja M. Do neurons have a reserve of sodium channels for the generation of action potentials? A study on acutely isolated CA1 neurons from the guinea-pig hippocampus. Eur J Neurosci 12: 1-7, 2000.

344. Maex R, De Schutter E. Resonant synchronization in heterogeneous networks of inhibitory neurons. J Neurosci 23: 10503-10514, 2003.

345. Magistretti J, Alonso A. Fine gating properties of channels responsible for persistent sodium current generation in entorhinal cortex neurons. J Gen Physiol 120: 855-873, 2002.

346. Mainen ZF, Joerges J, Huguenard JR, Sejnowski TJ. A model of spike initiation in neocortical pyramidal neurons. Neuron 15: 1427-1439, 1995.

347. Major G, Larkman AU, Jonas P, Sakmann B, Jack JJ. Detailed passive cable models of whole-cell recorded CA3 pyramidal neurons in rat hippocampal slices. J Neurosci 14: 4613-4638, 1994.
348. Manor Y, Gonczarowski J, Segev I. Propagation of action potentials along complex axonal trees. Model and implementation. Biophys J 60: 1411-1423, 1991.

349. Manor Y, Koch C, Segev I. Effect of geometrical irregularities on propagation delay in axonal trees. Biophys $J$ 60: 1424-1437, 1991.

350. Mar A, Drapeau P. Modulation of conduction block in leech mechanosensory neurons. J Neurosci 16: 4335-4343, 1996.

351. Marder E. Neurobiology: extending influence. Nature 441: 702 703, 2006.

352. Margrie TW, Brecht M, Sakmann B. In vivo, low-resistance, whole-cell recordings from neurons in the anaesthetized and awake mammalian brain. Pflügers Arch 444: 491-498, 2002.

353. Martina M, Jonas P. Functional differences in $\mathrm{Na}^{+}$channel gating between fast-spiking interneurones and principal neurones of rat hippocampus. J Physiol 505: 593-603, 1997.

354. Martina M, Vida I, Jonas P. Distal initiation and active propagation of action potentials in interneuron dendrites. Science 287: 295-300, 2000.

355. Mathy A, Ho SS, Davie JT, Duguid IC, Clark BA, Hausser M. Encoding of oscillations by axonal bursts in inferior olive neurons. Neuron 62: 388-399, 2009.

356. Maximov A, Bezprozvanny I. Synaptic targeting of N-type calcium channels in hippocampal neurons. J Neurosci 22: 6939-6952, 2002.

357. Maximov A, Sudhof TC, Bezprozvanny I. Association of neuronal calcium channels with modular adaptor proteins. J Biol Chem 274: 24453-24456, 1999.

358. McAlpine D, Grothe B. Sound localization and delay lines-do mammals fit the model? Trends Neurosci 26: 347-350, 2003.

359. McCormick DA, Shu Y, Yu Y. Neurophysiology: Hodgkin and Huxley model-still standing? Nature 445: E1-3, 2007.

360. McKinney RA, Debanne D, Gahwiler BH, Thompson SM. Lesion-induced axonal sprouting and hyperexcitability in the hippocampus in vitro: implications for the genesis of posttraumatic epilepsy. Nat Med 3: 990-996, 1997.

361. McNaughton BL, Shen J, Rao G, Foster TC, Barnes CA. Persistent increase of hippocampal presynaptic axon excitability after repetitive electrical stimulation: dependence on $N$-methyl-D-aspartate receptor activity, nitric-oxide synthase, and temperature. Proc Natl Acad Sci USA 91: 4830-4834, 1994.

362. Meeks JP, Jiang X, Mennerick S. Action potential fidelity during normal and epileptiform activity in paired soma-axon recordings from rat hippocampus. J Physiol 566: 425-441, 2005.

363. Meeks JP, Mennerick S. Action potential initiation and propagation in CA3 pyramidal axons. J Neurophysiol 97: 3460-3472, 2007.

364. Meeks JP, Mennerick S. Selective effects of potassium elevations on glutamate signaling and action potential conduction in hippocampus. J Neurosci 24: 197-206, 2004.

365. Mejia-Gervacio S, Collin T, Pouzat C, Tan YP, Llano I, Marty A. Axonal speeding: shaping synaptic potentials in small neurons by the axonal membrane compartment. Neuron 53: 843-855, 2007.

366. Meyrand P, Weimann JM, Marder E. Multiple axonal spike initiation zones in a motor neuron: serotonin activation. J Neurosci 12: 2803-2812, 1992.

367. Micu I, Jiang Q, Coderre E, Ridsdale A, Zhang L, Woulfe J, Yin X, Trapp BD, McRory JE, Rehak R, Zamponi GW, Wang W, Stys PK. NMDA receptors mediate calcium accumulation in myelin during chemical ischaemia. Nature 439: 988-992, 2006.

368. Middleton SJ, Racca C, Cunningham MO, Traub RD, Monyer H, Knopfel T, Schofield IS, Jenkins A, Whittington MA. Highfrequency network oscillations in cerebellar cortex. Neuron 58: 763-774, 2008.

369. Miledi R, Slater CR. The action of calcium on neuronal synapses in the squid. J Physiol 184: 473-498, 1966.

370. Mochida S, Westenbroek RE, Yokoyama CT, Zhong H, Myers SJ, Scheuer T, Itoh K, Catterall WA. Requirement for the synaptic protein interaction site for reconstitution of synaptic transmission by P/Q-type calcium channels. Proc Natl Acad Sci USA 100: 2819-2824, 2003.

371. Monaghan MM, Trimmer JS, Rhodes KJ. Experimental localization of $\mathrm{Kv1}$ family voltage-gated $\mathrm{K}^{+}$channel alpha and beta subunits in rat hippocampal formation. J Neurosci 21: 5973-5983, 2001. 
372. Monsivais P, Clark BA, Roth A, Hausser M. Determinants of action potential propagation in cerebellar Purkinje cell axons. $J$ Neurosci 25: 464-472, 2005.

373. Moore JW, Stockbridge N, Westerfield M. On the site of impulse initiation in a neurone. $J$ Physiol 336: 301-311, 1983.

374. Muschol M, Kosterin P, Ichikawa M, Salzberg BM. Activitydependent depression of excitability and calcium transients in the neurohypophysis suggests a model of "stuttering conduction." $J$ Neurosci 23: 11352-11362, 2003.

375. Nakada C, Ritchie K, Oba Y, Nakamura M, Hotta Y, Iino R, Kasai RS, Yamaguchi K, Fujiwara T, Kusumi A. Accumulation of anchored proteins forms membrane diffusion barriers during neuronal polarization. Nat Cell Biol 5: 626-632, 2003.

376. Nakamura M, Sekino Y, Manabe T. GABAergic interneurons facilitate mossy fiber excitability in the developing hippocampus. $J$ Neurosci 27: 1365-1373, 2007.

377. Nakamura Y, Takahashi T. Developmental changes in potassium currents at the rat calyx of Held presynaptic terminal. $J$ Physiol 581: 1101-1112, 2007.

378. Nashmi R, Jones OT, Fehlings MG. Abnormal axonal physiology is associated with altered expression and distribution of Kv1.1 and Kv1.2 K ${ }^{+}$channels after chronic spinal cord injury. Eur J Neurosci 12: 491-506, 2000.

379. Naundorf B, Wolf F, Volgushev M. Unique features of action potential initiation in cortical neurons. Nature 440: 1060-1063, 2006.

380. Nave KA. Myelination and the trophic support of long axons. Nat Rev Neurosci 11: 275-283, 2010.

381. Nave KA, Trapp BD. Axon-glial signaling and the glial support of axon function. Annu Rev Neurosci 31: 535-561, 2008.

382. Nicholls J, Wallace BG. Modulation of transmission at an inhibitory synapse in the central nervous system of the leech. $J$ Physiol 281: 157-170, 1978

383. Nishiyama H, Fukaya M, Watanabe M, Linden DJ. Axonal motility and its modulation by activity are branch-type specific in the intact adult cerebellum. Neuron 56: 472-487, 2007.

384. Noble D. Applications of Hodgkin-Huxley equations to excitable tissues. Physiol Rev 46: 1-50, 1966.

385. Noble D, Stein RB. The threshold conditions for initiation of action potentials by excitable cells. $J$ Physiol 187: 129-162, 1966.

386. Notomi T, Shigemoto R. Immunohistochemical localization of Ih channel subunits, HCN1-4, in the rat brain. J Comp Neurol 471: 241-276, 2004.

387. Nusser Z. Variability in the subcellular distribution of ion channels increases neuronal diversity. Trends Neurosci 32: 267-274, 2009.

388. O'Keefe J. Hippocampus, theta, and spatial memory. Curr Opin Neurobiol 3: 917-924, 1993.

389. Obaid AL, Salzberg BM. Micromolar 4-aminopyridine enhances invasion of a vertebrate neurosecretory terminal arborization: optical recording of action potential propagation using an ultrafast photodiode-MOSFET camera and a photodiode array. $J$ Gen Physiol 107: 353-368, 1996.

390. Obermair GJ, Szabo Z, Bourinet E, Flucher BE. Differential targeting of the L-type $\mathrm{Ca}^{2+}$ channel alpha $1 \mathrm{C}(\mathrm{CaV} 1.2)$ to synaptic and extrasynaptic compartments in hippocampal neurons. Eur $J$ Neurosci 19: 2109-2122, 2004.

391. Ogawa Y, Horresh I, Trimmer JS, Bredt DS, Peles E, Rasband MN. Postsynaptic density-93 clusters Kv1 channels at axon initial segments independently of Caspr2. J Neurosci 28: 5731-5739, 2008.

392. Ogawa Y, Oses-Prieto J, Kim MY, Horresh I, Peles E, Burlingame AL, Trimmer JS, Meijer D, Rasband MN. ADAM22, a Kv1 channel-interacting protein, recruits membrane-associated guanylate kinases to juxtaparanodes of myelinated axons. J Neurosci 30: 1038-1048, 2010.

393. Ogawa Y, Rasband MN. The functional organization and assembly of the axon initial segment. Curr Opin Neurobiol 18: 307-313, 2008.

394. Ogiwara I, Miyamoto H, Morita N, Atapour N, Mazaki E, Inoue I, Takeuchi T, Itohara S, Yanagawa Y, Obata K, Furuichi T, Hensch TK, Yamakawa K. $\mathrm{Na}(\mathrm{v}) 1.1$ localizes to axons of parvalbumin-positive inhibitory interneurons: a circuit basis for epileptic seizures in mice carrying an Scnla gene mutation. $J$ Neurosci 27: 5903-5914, 2007.
395. Palani D, Baginskas A, Raastad M. Bursts and hyperexcitability in non-myelinated axons of the rat hippocampus. Neuroscience 167: 1004-1013, 2010.

396. Palmer LM, Clark BA, Grundemann J, Roth A, Stuart GJ, Hausser M. Initiation of simple and complex spikes in cerebellar Purkinje cells. J Physiol 588: 1709-1717, 2010.

397. Palmer LM, Stuart GJ. Site of action potential initiation in layer 5 pyramidal neurons. $J$ Neurosci 26: 1854-1863, 2006.

398. Pan Z, Kao T, Horvath Z, Lemos J, Sul JY, Cranstoun SD, Bennett V, Scherer SS, Cooper EC. A common ankyrin-G-based mechanism retains KCNQ and NaV channels at electrically active domains of the axon. $J$ Neurosci 26: 2599-2613, 2006.

399. Pan Z, Selyanko AA, Hadley JK, Brown DA, Dixon JE, McKinnon D. Alternative splicing of KCNQ2 potassium channel transcripts contributes to the functional diversity of M-currents. $J$ Physiol 531: 347-358, 2001.

400. Paradiso K, Wu LG. Small voltage changes at nerve terminals travel up axons to affect action potential initiation. Nat Neurosci 12: 541-543, 2009.

401. Parnas I. Differential block at high frequency of branches of a single axon innervating two muscles. J Neurophysiol 35: 903-914, 1972.

402. Parnas I. Propagation in nonuniform neurites: form and function in axons. In: The Neurosciences, edited by Schmitt FO, Worden FG. Cambridge, MA: MIT Press, 1979, p. 499-512.

403. Parnas I, Hochstein S, Parnas H. Theoretical analysis of parameters leading to frequency modulation along an inhomogeneous axon. J Neurophysiol 39: 909-923, 1976.

404. Parnas I, Segev I. A mathematical model for conduction of action potentials along bifurcating axons. J Physiol 295: 323-343, 1979.

405. Perlson E, Maday S, Fu MM, Moughamian AJ, Holzbaur EL. Retrograde axonal transport: pathways to cell death? Trends Neurosci 33: 335-344, 2010.

406. Peters HC, Hu H, Pongs O, Storm JF, Isbrandt D. Conditional transgenic suppression of $\mathrm{M}$ channels in mouse brain reveals functions in neuronal excitability, resonance and behavior. Nat Neurosci 8: 51-60, 2005.

407. Pfaffmann C. Potentials in the isolated medullated axon. $J$ Cell Comp Physiol 16: 407-410, 1940.

408. Pietrobon D. Biological science of headache channels. Handb Clin Neurol 97: 73-83, 2010.

409. Pietrobon D. Insights into migraine mechanisms and CaV2.1 calcium channel function from mouse models of familial hemiplegic migraine. J Physiol 588: 1871-1878, 2010.

410. Pinault D. Backpropagation of action potentials generated at ectopic axonal loci: hypothesis that axon terminals integrate local environmental signals. Brain Res 21: 42-92, 1995.

411. Pinault D, Deschenes M. Projection and innervation patterns of individual thalamic reticular axons in the thalamus of the adult rat: a three-dimensional, graphic, morphometric analysis. J Comp Neurol 391: 180-203, 1998.

412. Pinheiro PS, Mulle C. Presynaptic glutamate receptors: physiological functions and mechanisms of action. Nat Rev Neurosci 9: 423-436, 2008.

413. Poliak S, Peles E. The local differentiation of myelinated axons at nodes of Ranvier. Nat Rev Neurosci 4: 968-980, 2003.

414. Poliak S, Salomon D, Elhanany H, Sabanay H, Kiernan B, Pevny L, Stewart CL, Xu X, Chiu SY, Shrager P, Furley AJ, Peles E. Juxtaparanodal clustering of Shaker-like $\mathrm{K}^{+}$channels in myelinated axons depends on Caspr2 and TAG-1. J Cell Biol 162: 1149-1160, 2003.

415. Ponce A, Bueno E, Kentros C, Vega-Saenz de Miera E, Chow A, Hillman D, Chen S, Zhu L, Wu MB, Wu X, Rudy B, Thornhill WB. G-protein-gated inward rectifier $\mathrm{K}^{+}$channel proteins (GIRK1) are present in the soma and dendrites as well as in nerve terminals of specific neurons in the brain. J Neurosci 16: 1990-2001, 1996.

416. Poolos NP, Mauk MD, Kocsis JD. Activity-evoked increases in extracellular potassium modulate presynaptic excitability in the CA1 region of the hippocampus. J Neurophysiol 58: 404-416, 1987.

417. Portera-Cailliau C, Weimer RM, De Paola V, Caroni P, Svoboda $\mathrm{K}$. Diverse modes of axon elaboration in the developing neocortex. PLoS Biol 3: e272, 2005. 
418. Pouille F, Scanziani M. Enforcement of temporal fidelity in pyramidal cells by somatic feed-forward inhibition. Science 293: 1159-1163, 2001.

419. Pouzat C, Marty A. Somatic recording of GABAergic autoreceptor current in cerebellar stellate and basket cells. J Neurosci 19: 1675-1690, 1999.

420. Pozo K, Goda Y. Unraveling mechanisms of homeostatic synaptic plasticity. Neuron 66: 337-351, 2010.

421. Prakriya M, Mennerick S. Selective depression of low-release probability excitatory synapses by sodium channel blockers. Neuron 26: 671-682, 2000.

422. Raastad M, Shepherd GM. Single-axon action potentials in the rat hippocampal cortex. J Physiol 548: 745-752, 2003.

423. Raffaelli G, Saviane C, Mohajerani MH, Pedarzani P, Cherubini E. BK potassium channels control transmitter release at CA3CA3 synapses in the rat hippocampus. J Physiol 557: 147-157, 2004.

424. Rall W. Branching dendritic trees and motoneuron membrane resistivity. Exp Neurol 1: 491-527, 1959.

425. Rall W. Distributions of potential in cylindrical coordinates and time constants for a membrane cylinder. Biophys $J$ 9: 1509-1541, 1969.

426. Rall W. Theoretical significance of dendritic tree for neural inputoutput relations. In: Neural Theory of Modeling, edited by Reiss FP. Palo Alto: Standford Univ. Press, 1964

427. Raman IM and Bean BP. Resurgent sodium current and action potential formation in dissociated cerebellar Purkinje neurons. $J$ Neurosci 17: 4517-4526, 1997

428. Ramon F, Joyner RW, Moore JW. Propagation of action potentials in inhomogeneous axon regions. Federation Proc 34: 13571363,1975

429. Ramon y Cajal S (Editor). Histologie du système nerveux de l'homme et des vertébrés. Paris: Maloine, 1911.

430. Ramon y Cajal S. Leyes de la morphologia y dinamismo de las células nerviosas. Rev Trim Micrograf 2: 1-12, 1897.

431. Rancz EA, Hausser M. Dendritic calcium spikes are tunable triggers of cannabinoid release and short-term synaptic plasticity in cerebellar Purkinje neurons. J Neurosci 26: 5428-5437, 2006.

432. Rancz EA, Ishikawa T, Duguid I, Chadderton P, Mahon S, Hausser M. High-fidelity transmission of sensory information by single cerebellar mossy fibre boutons. Nature 450: 1245-1248, 2007.

433. Ranvier LA (Editor). Leçons sur l'histologie du système nerveux. Paris: Savy, 1878.

434. Rapp M, Yarom Y, Segev I. Modeling back propagating action potential in weakly excitable dendrites of neocortical pyramidal cells. Proc Natl Acad Sci USA 93: 11985-11990, 1996.

435. Rasband MN, Trimmer JS. Subunit composition and novel localization of $\mathrm{K}^{+}$channels in spinal cord. J Comp Neurol 429: 166-176, 2001.

436. Rasminsky M. Ephaptic transmission between single nerve fibres in the spinal nerve roots of dystrophic mice. J Physiol 305: 151-169, 1980.

437. Redman RS, Silinsky EM. ATP released together with acetylcholine as the mediator of neuromuscular depression at frog motor nerve endings. J Physiol 477: 117-127, 1994.

438. Regehr W, Kehoe JS, Ascher P, Armstrong C. Synaptically triggered action potentials in dendrites. Neuron 11: 145-151, 1993.

439. Remak R. über den Bau der grauen Säuden im Rückenmark der Säugethiere. Deutsche Klinik 7: 295, 1855.

440. Reutskiy S, Rossoni E, Tirozzi B. Conduction in bundles of demyelinated nerve fibers: computer simulation. Biol Cybern 89: 439-448, 2003.

441. Reyes AD. Synchrony-dependent propagation of firing rate in iteratively constructed networks in vitro. Nat Neurosci 6: 593-599, 2003.

442. Rhee JS, Wang ZM, Nabekura J, Inoue K, Akaike N. ATP facilitates spontaneous glycinergic IPSC frequency at dissociated rat dorsal horn interneuron synapses. J Physiol 524: 471-483, 2000.

443. Rhodes KJ, Strassle BW, Monaghan MM, Bekele-Arcuri Z, Matos MF, Trimmer JS. Association and colocalization of the Kvbeta1 and Kvbeta2 beta-subunits with Kv1 alpha-subunits in mammalian brain $\mathrm{K}^{+}$channel complexes. J Neurosci 17: 82468258, 1997.
444. Ritchie JM. On the relation between fibre diameter and conduction velocity in myelinated nerve fibres. Proc R Soc Lond B Biol Sci 217: 29-35, 1982.

445. Ritchie JM, Straub RW. The after-effects of repetitive stimulation on mammalian non-medullated fibres. J Physiol 134: 698-711, 1956.

446. Ritchie JM, Straub RW. The hyperpolarization which follows activity in mammalian non-medullated fibres. J Physiol 136: 80-97, 1957.

447. Roncarati R, Di Chio M, Sava A, Terstappen GC, Fumagalli G. Presynaptic localization of the small conductance calcium-activated potassium channel SK3 at the neuromuscular junction. Neuroscience 104: 253-262, 2001.

448. Roopun AK, Middleton SJ, Cunningham MO, LeBeau FE, Bibbig A, Whittington MA, Traub RD. A beta2-frequency $(20-30 \mathrm{~Hz})$ oscillation in nonsynaptic networks of somatosensory cortex. Proc Natl Acad Sci USA 103: 15646-15650, 2006.

449. Royeck M, Horstmann MT, Remy S, Reitze M, Yaari Y, Beck H. Role of axonal NaV1.6 sodium channels in action potential initiation of CA1 pyramidal neurons. J Neurophysiol 100: 2361-2380, 2008.

450. Rudomin P, Schmidt RF. Presynaptic inhibition in the vertebrate spinal cord revisited. Exp Brain Res 129: 1-37, 1999.

451. Ruiz A, Campanac E, Scott RS, Rusakov DA, Kullmann DM. Presynaptic $\mathrm{GABA}_{\mathrm{A}}$ receptors enhance transmission and LTP induction at hippocampal mossy fiber synapses. Nat Neurosci 13: 431-438, 2010.

452. Ruiz A, Fabian-Fine R, Scott R, Walker MC, Rusakov DA, Kullmann DM. $\mathrm{GABA}_{\mathrm{A}}$ receptors at hippocampal mossy fibers. Neuron 39: 961-973, 2003.

453. Rushton WA. A theory of the effects of fibre size in medullated nerve. J Physiol 115: 101-122, 1951.

454. Sabatini BL, Regehr WG. Timing of neurotransmission at fast synapses in the mammalian brain. Nature 384: 170-172, 1996.

455. Sailer CA, Kaufmann WA, Kogler M, Chen L, Sausbier U, Ottersen OP, Ruth P, Shipston MJ, Knaus HG. Immunolocalization of BK channels in hippocampal pyramidal neurons. Eur $J$ Neurosci 24: 442-454, 2006.

456. Sakatani K, Chesler M, Hassan AZ. GABA $_{\mathrm{A}}$ receptors modulate axonal conduction in dorsal columns of neonatal rat spinal cord. Brain Res 542: 273-279, 1991.

457. Sakatani K, Chesler M, Hassan AZ, Lee M, Young W. Nonsynaptic modulation of dorsal column conduction by endogenous GABA in neonatal rat spinal cord. Brain Res 622: 43-50, 1993.

458. Sanchez-Ponce D, Tapia M, Munoz A, Garrido JJ. New role of IKK alpha/beta phosphorylated I kappa B alpha in axon outgrowth and axon initial segment development. Mol Cell Neurosci 37: 832$844,2008$.

459. Santoro B, Grant SG, Bartsch D, Kandel ER. Interactive cloning with the SH3 domain of N-src identifies a new brain specific ion channel protein, with homology to eag and cyclic nucleotide-gated channels. Proc Natl Acad Sci USA 94: 14815-14820, 1997.

460. Saviane C, Mohajerani MH, Cherubini E. An ID-like current that is downregulated by $\mathrm{Ca}^{2+}$ modulates information coding at CA3CA3 synapses in the rat hippocampus. J Physiol 552: 513-524, 2003.

461. Schafer DP, Jha S, Liu F, Akella T, McCullough LD, Rasband MN. Disruption of the axon initial segment cytoskeleton is a new mechanism for neuronal injury. J Neurosci 29: 13242-13254, 2009.

462. Schiller J, Schiller Y, Stuart G, Sakmann B. Calcium action potentials restricted to distal apical dendrites of rat neocortical pyramidal neurons. J Physiol 505: 605-616, 1997.

463. Schmidt-Hieber C, Jonas P, Bischofberger J. Action potential initiation and propagation in hippocampal mossy fibre axons. $J$ Physiol 586: 1849-1857, 2008.

464. Schmitz D, Schuchmann S, Fisahn A, Draguhn A, Buhl EH, Petrasch-Parwez E, Dermietzel R, Heinemann U, Traub RD. Axo-axonal coupling: a novel mechanism for ultrafast neuronal communication. Neuron 31: 831-840, 2001.

465. Schneidman E, Freedman B, Segev I. Ion channel stochasticity may be critical in determining the reliability and precision of spike timing. Neural Comput 10: 1679-1703, 1998.

466. Schroeder BC, Kubisch C, Stein V, Jentsch TJ. Moderate loss of function of cyclic-AMP-modulated KCNQ2/KCNQ3 $\mathrm{K}^{+}$channels causes epilepsy. Nature 396: 687-690, 1998. 
467. Scott LL, Hage TA, Golding NL. Weak action potential backpropagation is associated with high-frequency axonal firing capability in principal neurons of the gerbil medial superior olive. $J$ Physiol 583: 647-661, 2007.

468. Scott R, Ruiz A, Henneberger C, Kullmann DM, Rusakov DA. Analog modulation of mossy fiber transmission is uncoupled from changes in presynaptic $\mathrm{Ca}^{2+}$. J Neurosci 28: 7765-7773, 2008.

469. Segev I. Computer study of presynaptic inhibition controlling the spread of action potentials into axonal terminals. J Neurophysiol 63: 987-998, 1990

470. Segev I, Schneidman E. Axons as computing devices: basic insights gained from models. J Physiol 93: 263-270, 1999.

471. Sekerli M, Del Negro CA, Lee RH, Butera RJ. Estimating action potential thresholds from neuronal time-series: new metrics and evaluation of methodologies. IEEE Trans Biomed Eng 51: 1665$1672,2004$.

472. Semyanov A, Kullmann DM. Kainate receptor-dependent axonal depolarization and action potential initiation in interneurons. Nat Neurosci 4: 718-723, 2001.

473. Shah MM, Migliore M, Valencia I, Cooper EC, Brown DA. Functional significance of axonal Kv7 channels in hippocampal pyramidal neurons. Proc Natl Acad Sci USA 105: 7869-7874, 2008.

474. Shah MM, Mistry M, Marsh SJ, Brown DA, Delmas P. Molecular correlates of the M-current in cultured rat hippocampal neurons. J Physiol 544: 29-37, 2002.

475. Shapiro E, Castellucci VF, Kandel ER. Presynaptic membrane potential affects transmitter release in an identified neuron in Aplysia by modulating the $\mathrm{Ca}^{2+}$ and $\mathrm{K}^{+}$currents. Proc Natl Acad Sci USA 77: 629-633, 1980.

476. Sheffield M, Best T, Mensh BD, Kath W, Spruston N. Slow integration leads to persistent action potential firing in distal axons of coupled interneurons. Nat Neurosci 14: 200-207, 2011.

477. Sheng M, Liao YJ, Jan YN, Jan LY. Presynaptic A-current based on heteromultimeric $\mathrm{K}^{+}$channels detected in vivo. Nature 365: $72-75,1993$

478. Sheng M, Tsaur ML, Jan YN, Jan LY. Subcellular segregation of two A-type $\mathrm{K}^{+}$channel proteins in rat central neurons. Neuron 9 : 271-284, 1992.

479. Sheng ZH, Rettig J, Takahashi M, Catterall WA. Identification of a syntaxin-binding site on N-type calcium channels. Neuron 13: 1303-1313, 1994.

480. Shepherd GM (Editor) Foundations of the Neuron Doctrine. New York: Oxford Univ. Press, 1991.

481. Shepherd GM, Raastad M. Axonal varicosity distributions along parallel fibers: a new angle on a cerebellar circuit. Cerebellum 2: $110-113,2003$.

482. Shepherd GM, Raastad M, Andersen P. General and variable features of varicosity spacing along unmyelinated axons in the hippocampus and cerebellum. Proc Natl Acad Sci USA 99: 63406345,2002

483. Sherman DL, Brophy PJ. Mechanisms of axon ensheathment and myelin growth. Nat Rev Neurosci 6: 683-690, 2005.

484. Shimahara T. Modulation of synaptic output by the transient outward potassium current in Aplysia. Neurosci Lett 24: 139-142, 1981.

485. Shimahara T. Presynaptic modulation of transmitter release by the early outward potassium current in Aplysia. Brain Res 263: 51-56, 1983 .

486. Shimahara T, Peretz B. Soma potential of an interneurone controls transmitter release in a monosynaptic pathway in Aplysia. Nature 273: 158-160, 1978.

487. Shimahara T, Tauc L. Multiple interneuronal afferents to the giant cells in Aplysia. J Physiol 247: 299-319, 1975.

488. Shu Y, Duque A, Yu Y, Haider B, McCormick DA. Properties of action-potential initiation in neocortical pyramidal cells: evidence from whole cell axon recordings. $J$ Neurophysiol 97: 746-760, 2007.

489. Shu Y, Hasenstaub A, Duque A, Yu Y, McCormick DA. Modulation of intracortical synaptic potentials by presynaptic somatic membrane potential. Nature 441: 761-765, 2006.

490. Shu Y, Yu Y, Yang J, McCormick DA. Selective control of cortical axonal spikes by a slowly inactivating $\mathrm{K}^{+}$current. Proc Natl Acad Sci USA 104: 11453-11458, 2007.
491. Singer W. Neuronal synchrony: a versatile code for the definition of relations? Neuron 24: 49-65, 1999.

492. Singh NA, Charlier C, Stauffer D, DuPont BR, Leach RJ, Melis R, Ronen GM, Bjerre I, Quattlebaum T, Murphy JV, McHarg ML, Gagnon D, Rosales TO, Peiffer A, Anderson VE, Leppert M. A novel potassium channel gene, $\mathrm{KCNQ} 2$, is mutated in an inherited epilepsy of newborns. Nat Genet 18: 25-29, 1998.

493. Sjostrom PJ, Rancz EA, Roth A, Hausser M. Dendritic excitability and synaptic plasticity. Physiol Rev 88: 769-840, 2008.

494. Sjostrom PJ, Turrigiano GG, Nelson SB. Neocortical LTD via coincident activation of presynaptic NMDA and cannabinoid receptors. Neuron 39: 641-654, 2003.

495. Smart SL, Lopantsev V, Zhang CL, Robbins CA, Wang H, Chiu SY, Schwartzkroin PA, Messing A, Tempel BL. Deletion of the $\mathrm{K}(\mathrm{V}) 1.1$ potassium channel causes epilepsy in mice. Neuron 20: 809-819, 1998

496. Smith DO. Mechanisms of action potential propagation failure at sites of axon branching in the crayfish. J Physiol 301: 243-259, 1980.

497. Smith DO. Morphological aspects of the safety factor for action potential propagation at axon branch points in the crayfish. $J$ Physiol 301: 261-269, 1980.

498. Soleng AF, Chiu K, Raastad M. Unmyelinated axons in the rat hippocampus hyperpolarize and activate an $\mathrm{H}$ current when spike frequency exceeds $1 \mathrm{~Hz}$. J Physiol 552: 459-470, 2003.

499. Somogyi P, Freund TF, Cowey A. The axo-axonic interneuron in the cerebral cortex of the rat, cat and monkey. Neuroscience 7: 2577-2607, 1982.

500. Somogyi P, Nunzi MG, Gorio A, Smith AD. A new type of specific interneuron in the monkey hippocampus forming synapses exclusively with the axon initial segments of pyramidal cells. Brain Res 259: 137-142, 1983.

501. Somogyi P, Smith AD, Nunzi MG, Gorio A, Takagi H, Wu JY. Glutamate decarboxylase immunoreactivity in the hippocampus of the cat: distribution of immunoreactive synaptic terminals with special reference to the axon initial segment of pyramidal neurons. J Neurosci 3: 1450-1468, 1983.

502. Song AH, Wang D, Chen G, Li Y, Luo J, Duan S, Poo MM. A selective filter for cytoplasmic transport at the axon initial segment. Cell 136: 1148-1160, 2009.

503. Sourdet V, Russier M, Daoudal G, Ankri N, Debanne D. Longterm enhancement of neuronal excitability and temporal fidelity mediated by metabotropic glutamate receptor subtype 5. J Neurosci 23: 10238-10248, 2003.

504. Southan AP, Robertson B. Electrophysiological characterization of voltage-gated $\mathrm{K}^{+}$currents in cerebellar basket and purkinje cells: Kv1 and Kv3 channel subfamilies are present in basket cell nerve terminals. $J$ Neurosci 20: 114-122, 2000.

505. Spampanato J, Escayg A, Meisler MH, Goldin AL. Functional effects of two voltage-gated sodium channel mutations that cause generalized epilepsy with febrile seizures plus type 2 . $J$ Neurosci 21: 7481-7490, 2001

506. Spruston N, Schiller Y, Stuart G, Sakmann B. Activity-dependent action potential invasion and calcium influx into hippocampal CA1 dendrites. Science 268: 297-300, 1995.

507. Stanford LR. Conduction velocity variations minimize conduction time differences among retinal ganglion cell axons. Science 238: $358-360,1987$.

508. Stettler DD, Yamahachi H, Li W, Denk W, Gilbert CD. Axons and synaptic boutons are highly dynamic in adult visual cortex. Neuron 49: 877-887, 2006.

509. Stevens B, Fields RD. Response of Schwann cells to action potentials in development. Science 287: 2267-2271, 2000.

510. Streit J, Luscher C, Luscher HR. Depression of postsynaptic potentials by high-frequency stimulation in embryonic motoneurons grown in spinal cord slice cultures. J Neurophysiol 68: 17931803,1992

511. Stuart G, Hausser M. Initiation and spread of sodium action potentials in cerebellar Purkinje cells. Neuron 13: 703-712, 1994.

512. Stuart G, Sakmann B. Amplification of EPSPs by axosomatic sodium channels in neocortical pyramidal neurons. Neuron 15 : 1065-1076, 1995. 
513. Stuart G, Schiller J, Sakmann B. Action potential initiation and propagation in rat neocortical pyramidal neurons. J Physiol 505: 617-632, 1997

514. Stuart G, Spruston N, Sakmann B, Hausser M. Action potential initiation and backpropagation in neurons of the mammalian CNS. Trends Neurosci 20: 125-131, 1997.

515. Stuart GJ, Redman SJ. The role of $\mathrm{GABA}_{\mathrm{A}}$ and $\mathrm{GABA}_{\mathrm{B}}$ receptors in presynaptic inhibition of Ia EPSPs in cat spinal motoneurones. $J$ Physiol 447: 675-692, 1992.

516. Stuart GJ, Sakmann B. Active propagation of somatic action potentials into neocortical pyramidal cell dendrites. Nature 367: 69-72, 1994.

517. Su H, Sochivko D, Becker A, Chen J, Jiang Y, Yaari Y, Beck H. Upregulation of a T-type $\mathrm{Ca}^{2+}$ channel causes a long-lasting modification of neuronal firing mode after status epilepticus. J Neurosci 22: 3645-3655, 2002.

518. Sugihara I, Lang EJ, Llinas R. Uniform olivocerebellar conduction time underlies Purkinje cell complex spike synchronicity in the rat cerebellum. J Physiol 470: 243-271, 1993.

519. Suter U, Scherer SS. Disease mechanisms in inherited neuropathies. Nat Rev Neurosci 4: 714-726, 2003.

520. Swadlow HA. Impulse conduction in the mammalian brain: physiological properties of individual axons monitored for several months. Science 218: 911-913, 1982.

521. Swadlow HA, Kocsis JD, Waxman SG. Modulation of impulse conduction along the axonal tree. Annu Rev Biophys Bioeng 9: 143-179, 1980.

522. Szabadics J, Varga C, Molnar G, Olah S, Barzo P, Tamas G. Excitatory effect of GABAergic axo-axonic cells in cortical microcircuits. Science 311: 233-235, 2006.

523. Tahvildari B, Yu Y, McCormick D. A novel form of plasticity: spontaneous repetitive action potential generation in cortical NPY interneurons results in ectopic spike initiation. In: 2010 Neuroscience Meeting Planner. San Diego, CA: Soc Neurosci, 2010.

524. Takeuchi A, Takeuchi N. Electrical changes in pre- and postsynaptic axons of the giant synapse of Loligo. J Gen Physiol 45: 1181-1193, 1962.

525. Tan YP, Llano I. Modulation by $\mathrm{K}^{+}$channels of action potentialevoked intracellular $\mathrm{Ca}^{2+}$ concentration rises in rat cerebellar basket cell axons. J Physiol 520: 65-78, 1999.

526. Tanaka H, Ma J, Tanaka KF, Takao K, Komada M, Tanda K, Suzuki A, Ishibashi T, Baba H, Isa T, Shigemoto R, Ono K, Miyakawa T, Ikenaka K. Mice with altered myelin proteolipid protein gene expression display cognitive deficits accompanied by abnormal neuron-glia interactions and decreased conduction velocities. J Neurosci 29: 8363-8371, 2009.

527. Tasaki I, Takeuchi T. Der am Ranvierschen Knoten entstehende Aktionsstrom und seine Bedeutung für die Erregungleitung. Pflügers Arch 244: 696-711, 1941.

528. Thio LL, Yamada KA. Differential presynaptic modulation of excitatory and inhibitory autaptic currents in cultured hippocampal neurons. Brain Res 1012: 22-28, 2004.

529. Thompson SM, Haas HL, Gahwiler BH. Comparison of the actions of adenosine at pre- and postsynaptic receptors in the rat hippocampus in vitro. J Physiol 451: 347-363, 1992.

530. Tian B, Cohen-Karni T, Qing Q, Duan X, Xie P, Lieber CM. Three-dimensional, flexible nanoscale field-effect transistors as localized bioprobes. Science 329: 830-834, 2010.

531. Tippens AL, Pare JF, Langwieser N, Moosmang S, Milner TA, Smith Y, Lee A. Ultrastructural evidence for pre- and postsynaptic localization of Cav1.2 L-type $\mathrm{Ca}^{2+}$ channels in the rat hippocampus. J Comp Neurol 506: 569-583, 2008.

532. Trapp BD, Nave KA. Multiple sclerosis: an immune or neurodegenerative disorder? Annu Rev Neurosci 31: 247-269, 2008.

533. Traub RD, Draguhn A, Whittington MA, Baldeweg T, Bibbig A, Buhl EH, Schmitz D. Axonal gap junctions between principal neurons: a novel source of network oscillations, perhaps epileptogenesis. Rev Neurosci 13: 1-30, 2002

534. Traub RD, Middleton SJ, Knopfel T, Whittington MA. Model of very fast $(>75 \mathrm{~Hz})$ network oscillations generated by electrical coupling between the proximal axons of cerebellar Purkinje cells. Eur J Neurosci 28: 1603-1616, 2008.
535. Trigo FF, Bouhours B, Rostaing P, Papageorgiou G, Corrie JE, Triller A, Ogden D, Marty A. Presynaptic miniature GABAergic currents in developing interneurons. Neuron 66: 235-247, 2010.

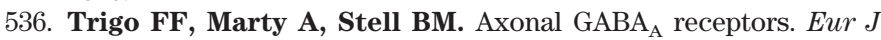
Neurosci 28: 841-848, 2008.

537. Turecek R, Trussell LO. Presynaptic glycine receptors enhance transmitter release at a mammalian central synapse. Nature 411: 587-590, 2001.

538. Turecek R, Trussell LO. Reciprocal developmental regulation of presynaptic ionotropic receptors. Proc Natl Acad Sci USA 99: 13884-13889, 2002.

539. Turrigiano GG, Nelson SB. Homeostatic plasticity in the developing nervous system. Nat Rev Neurosci 5: 97-107, 2004.

540. Vacher H, Mohapatra DP, Trimmer JS. Localization and targeting of voltage-dependent ion channels in mammalian central neurons. Physiol Rev 88: 1407-1447, 2008.

541. Van Essen DC. The contribution of membrane hyperpolarization to adaptation and conduction block in sensory neurones of the leech. J Physiol 230: 509-534, 1973.

542. Van Wart A, Trimmer JS, Matthews G. Polarized distribution of ion channels within microdomains of the axon initial segment. $J$ Comp Neurol 500: 339-352, 2007.

543. Veh RW, Lichtinghagen R, Sewing S, Wunder F, Grumbach IM, Pongs O. Immunohistochemical localization of five members of the Kv1 channel subunits: contrasting subcellular locations and neuron-specific co-localizations in rat brain. Eur $J$ Neurosci 7: 2189-2205, 1995.

544. Velte TJ, Masland RH. Action potentials in the dendrites of retinal ganglion cells. J Neurophysiol 81: 1412-1417, 1999.

545. Verdier D, Lund JP, Kolta A. GABAergic control of action potential propagation along axonal branches of mammalian sensory neurons. J Neurosci 23: 2002-2007, 2003.

546. Vervaeke K, Gu N, Agdestein C, Hu H, Storm JF. Kv7/KCNQ/ M-channels in rat glutamatergic hippocampal axons and their role in regulation of excitability and transmitter release. J Physiol 576: 235-256, 2006.

547. Vigot R, Barbieri S, Brauner-Osborne H, Turecek R, Shigemoto R, Zhang YP, Lujan R, Jacobson LH, Biermann B, Fritschy JM, Vacher CM, Muller M, Sansig G, Guetg N, Cryan JF, Kaupmann K, Gassmann M, Oertner TG, Bettler B. Differential compartmentalization and distinct functions of $\mathrm{GABA}_{\mathrm{B}}$ receptor variants. Neuron 50: 589-601, 2006.

548. Virchow R. über das ausgebreitet Vorkommen einer dem Nervenmark analogen Susbtanz in den tierischen Geweben. Virchows Arch Pathol Anat 6: 562, 1854.

549. Wall PD. Do nerve impulses penetrate terminal arborizations? A pre-presynaptic control mechanism. Trends Neurosci 18: 99-103, 1995.

550. Wang H, Kunkel DD, Martin TM, Schwartzkroin PA, Tempel BL. Heteromultimeric $\mathrm{K}^{+}$channels in terminal and juxtaparanodal regions of neurons. Nature 365: 75-79, 1993.

551. Wang H, Kunkel DD, Schwartzkroin PA, Tempel BL. Localization of Kv1.1 and Kv1.2, two K channel proteins, to synaptic terminals, somata, and dendrites in the mouse brain. J Neurosci 14: 4588-4599, 1994.

552. Wang LY, Kaczmarek LK. High-frequency firing helps replenish the readily releasable pool of synaptic vesicles. Nature 394: $384-$ 388, 1998.

553. Wang SS, Shultz JR, Burish MJ, Harrison KH, Hof PR, Towns LC, Wagers MW, Wyatt KD. Functional trade-offs in white matter axonal scaling. J Neurosci 28: 4047-4056, 2008.

554. Watanabe K, Morishima Y, Toigawa M, Hata Y. Experiencedriven axon retraction in the pharmacologically inactivated visual cortex does not require synaptic transmission. PLoS One 4: e4193, 2009.

555. Waxman SG. Axonal conduction and injury in multiple sclerosis: the role of sodium channels. Nat Rev Neurosci 7: 932-941, 2006.

556. Weiser M, Bueno E, Sekirnjak C, Martone ME, Baker H, Hillman D, Chen S, Thornhill W, Ellisman M, Rudy B. The potassium channel subunit KV3.1b is localized to somatic and axonal membranes of specific populations of CNS neurons. $J \mathrm{Neu}$ rosci 15: 4298-4314, 1995. 
557. Weragoda RM, Ferrer E, Walters ET. Memory-like alterations in Aplysia axons after nerve injury or localized depolarization. $J$ Neurosci 24: 10393-10401, 2004.

558. Westenbroek RE, Merrick DK, Catterall WA. Differential subcellular localization of the RI and RII $\mathrm{Na}^{+}$channel subtypes in central neurons. Neuron 3: 695-704, 1989.

559. Westrum LE, Blackstad TW. An electron microscopic study of the stratum radiatum of the rat hippocampus (regio superior, CA 1) with particular emphasis on synaptology. J Comp Neurol 119: 281-309, 1962.

560. White JA, Rubinstein JT, Kay AR. Channel noise in neurons. Trends Neurosci 23: 131-137, 2000.

561. Williams SR, Stuart GJ. Mechanisms and consequences of action potential burst firing in rat neocortical pyramidal neurons. $J$ Physiol 521: 467-482, 1999.

562. Wimmer VC, Reid CA, So EY, Berkovic SF, Petrou S. Axon initial segment dysfunction in epilepsy. J Physiol 588: 1829-1840, 2010.

563. Winckler B, Forscher P, Mellman I. A diffusion barrier maintains distribution of membrane proteins in polarized neurons. Nature 397: 698-701, 1999.

564. Wollner DA, Catterall WA. Localization of sodium channels in axon hillocks and initial segments of retinal ganglion cells. Proc Natl Acad Sci USA 83: 8424-8428, 1986.

565. Wong RK, Prince DA, Basbaum AI. Intradendritic recordings from hippocampal neurons. Proc Natl Acad Sci USA 76: 986-990, 1979 .

566. Woodruff AR, Monyer H, Sah P. GABAergic excitation in the basolateral amygdala. J Neurosci 26: 11881-11887, 2006.

567. Xiao S, Jan LY. A gate keeper for axonal transport. Cell 136: 996-998, 2009.

568. Xu J, Kang N, Jiang L, Nedergaard M, Kang J. Activity-dependent long-term potentiation of intrinsic excitability in hippocampal CA1 pyramidal neurons. J Neurosci 25: 1750-1760, 2005.

569. Xu K, Terakawa S. Fenestration nodes and the wide submyelinic space form the basis for the unusually fast impulse conduction of shrimp myelinated axons. J Exp Biol 202: 1979-1989, 1999.

570. Yamazaki Y, Hozumi Y, Kaneko K, Sugihara T, Fujii S, Goto K, Kato H. Modulatory effects of oligodendrocytes on the conduction velocity of action potentials along axons in the alveus of the rat hippocampal CA1 region. Neuron Glia Biol 3: 325-334, 2007.

571. Yang Y, Wang Y, Li S, Xu Z, Li H, Ma L, Fan J, Bu D, Liu B, Fan Z, Wu G, Jin J, Ding B, Zhu X, Shen Y. Mutations in SCN9A, encoding a sodium channel alpha subunit, in patients with primary erythermalgia. J Med Genet 41: 171-174, 2004.
572. Yau KW. Receptive fields, geometry and conduction block of sensory neurones in the central nervous system of the leech. J Physiol 263: 513-538, 1976.

573. Ye JH, Wang F, Krnjevic K, Wang W, Xiong ZG, Zhang J. Presynaptic glycine receptors on GABAergic terminals facilitate discharge of dopaminergic neurons in ventral tegmental area. $J$ Neurosci 24: 8961-8974, 2004.

574. Yoon KW, Rothman SM. Adenosine inhibits excitatory but not inhibitory synaptic transmission in the hippocampus. J Neurosci 11: 1375-1380, 1991.

575. Young JZ. The giant nerve fibres and epistellar body of cephalopods. Q J Microsc Sci 78: 367-386, 1936.

576. Yu FH, Mantegazza M, Westenbroek RE, Robbins CA, Kalume F, Burton KA, Spain WJ, McKnight GS, Scheuer T, Catterall WA. Reduced sodium current in GABAergic interneurons in a mouse model of severe myoclonic epilepsy in infancy. Nat Neurosci 9: 1142-1149, 2006.

577. Yu Y, Maureira C, Liu X, McCormick D. P/Q and N channels control baseline and spike-triggered calcium levels in neocortical axons and synaptic boutons. J Neurosci 30: 11858-11869, 2010.

578. Yu Y, Shu Y, McCormick DA. Cortical action potential backpropagation explains spike threshold variability and rapid-onset kinetics. J Neurosci 28: 7260-7272, 2008.

579. Yue C, Yaari Y. Axo-somatic and apical dendritic Kv7/M channels differentially regulate the intrinsic excitability of adult rat CA1 pyramidal cells. J Neurophysiol 95: 3480-3495, 2006.

580. Zecevic D. Multiple spike-initiation zones in single neurons revealed by voltage-sensitive dyes. Nature 381: 322-325, 1996.

581. Zhang SJ, Jackson MB. GABA-activated chloride channels in secretory nerve endings. Science 259: 531-534, 1993.

582. Zhang W, Linden DJ. The other side of the engram: experiencedriven changes in neuronal intrinsic excitability. Nat Rev Neurosci 4: 885-900, 2003.

583. Zhou L, Chiu SY. Computer model for action potential propagation through branch point in myelinated nerves. J Neurophysiol 85: 197-210, 2001.

584. Zhou L, Messing A, Chiu SY. Determinants of excitability at transition zones in Kv1.1-deficient myelinated nerves. J Neurosci 19: 5768-5781, 1999.

585. Zhou L, Zhang CL, Messing A, Chiu SY. Temperature-sensitive neuromuscular transmission in Kv1.1 null mice: role of potassium channels under the myelin sheath in young nerves. J Neurosci 18 : 7200-7215, 1998.

586. Ziskin JL, Nishiyama A, Rubio M, Fukaya M, Bergles DE. Vesicular release of glutamate from unmyelinated axons in white matter. Nat Neurosci 10: 321-330, 2007. 